\title{
LIGHT EXTRACTION ENHANCEMENT OF GAN BASED LEDS USING TOP GRATINGS, PATTERNED SAPPHIRE SUBSTRATES, AND REFLECTIVE SURFACES
}

\author{
A Thesis Presented to the \\ Electrical Engineering Department Faculty of \\ California Polytechnic State University, San Luis Obispo
}

\author{
In Partial Fulfillment \\ of the Requirements for the Degree \\ Master of Science in Electrical Engineering
}

by

Gregory James Chavoor

April 2012 
(C) 2012

Gregory James Chavoor

ALL RIGHTS RESERVED 


\section{COMMITTEE MEMBERSHIP}

TITLE:

AUTHOR:

DATE SUBMITTED:

Dr. Xiaomin Jin.

Committee Chair

Dr. David Braun

Committee Member

Dr. Robert Echols.

Committee Member

\section{LIGHT EXTRACTION ENHANCEMENT OF GAN BASED LEDS USING TOP GRATINGS, PATTERNED SAPPHIRE SUBSTRATES, AND REFLECTIVE SURFACES}

Gregory James Chavoor

April 2012
Signature

Signature 


\section{ABSTRACT \\ LIGHT EXTRACTION ENHANCEMENT OF GAN BASED LEDS USING TOP GRATINGS, PATTERNED SAPPHIRE SUBSTRATES, AND REFLECTIVE SURFACES}

\section{Gregory James Chavoor}

In the last 15 years, an immense amount of research has gone into developing high efficiency Gallium Nitride based light emitting diodes (LED). These devices have become increasingly popular in LED displays and solid state lighting. Due to the large difference in refractive index between GaN and Air, a significant amount of light reflects at the boundary and does not escape the device. This drawback decreases external quantum efficiency (EQE) by minimizing light extraction. Scientists and engineers continue to develop creative solutions to enhance light extraction. Some solutions include surface roughening, patterned sapphire substrates, and reflective layers.

This study proposes to increase external quantum efficiency and optimize light extraction efficiency of several LED structures using finite difference time domain analysis (FDTD). The structures under investigation include GaN based LEDs with nanoscale top gratings, patterned sapphire substrates in combination with $\mathrm{SiO}_{2}$ nanorod arrays, and reflective surfaces below and above the sapphire substrate. First, we optimize GaN based nanoscale top gratings and increase light extraction by $17.8 \%$. Next, we simulate ITO based top gratings and enhance light extraction by $40 \%$. Third, we optimize patterned sapphire substrate period and width and the vertical position of a $\mathrm{SiO}_{2}$ nanorod array. We achieve as high as $51.8 \%$ improvement in light extraction. Finally, we increase light extraction by $160 \%$ with the use of a silver reflection layer. 


\section{ACKNOWLEDGEMENTS}

First I would like to thank Professor Jin for welcoming me into a new area of research. As an undergraduate I had no preexisting knowledge on GaN-based semiconductors. Thanks to her confidence in my ability to learn a subject I have written this thesis along with two conference papers. Thank you for believing in me and pushing me beyond my limits.

Second, I want to thank my lovely wife Tracie for supporting me through all my educational endeavors. Your kindness, patience, and confidence in me never seem to end.

I would also like to thank Peking University for working along side me and hosting me for a summer in Beijing. China holds a special place in my heart and I am sure to be back again.

Finally, a special thanks to Dr. Braun and Dr. Echols (A.K.A Dr. Bob) for being a part of my committee. You both served a special role in teaching me the semi-conductor basics. I admire both of your commitment to teaching. Thank you.

This project is supported by 1) NSF Grant OISE Award \#1029135 from year 2010 to 2013 and 2) Chinese National Key Research Lab Collaboration Grant 2011-2012, Peking University in China. 


\section{TABLE OF CONTENTS}

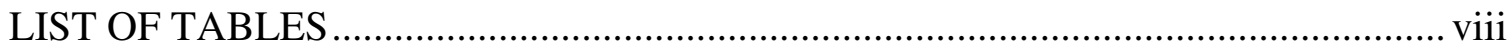

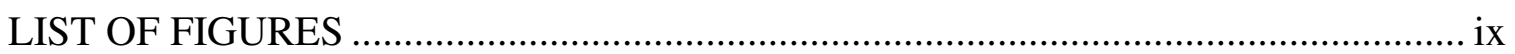

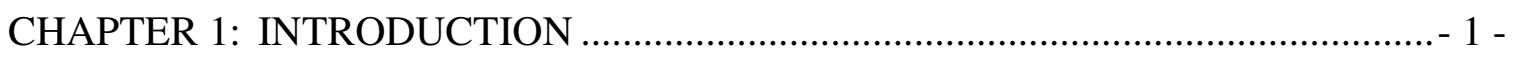

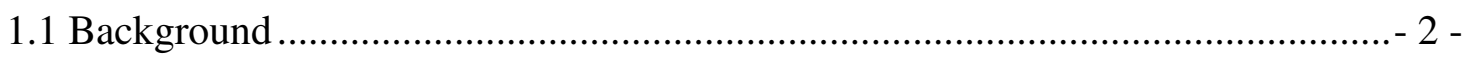

1.2 Disadvantages of GaN and Potential Solutions .................................................. 4 -

CHAPTER 2: RSOFT Fullwave analysis ................................................................... 9 -

2.1 Defining Materials and Grid Size ………………...............................................

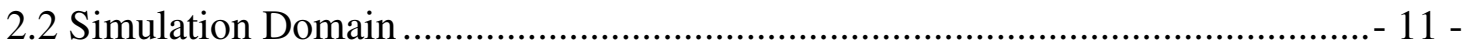

CHAPTER 3: NANOSCALE TOP TRANSMISSION GRATING …………….......... 14 -

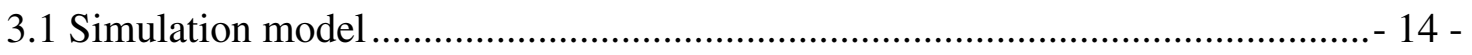

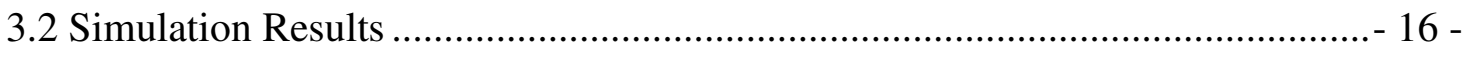

3.2.1 Cylindrical and conical gratings ................................................................ 16 -

3.2.2 Changing the fill factor .......................................................................... 19 -

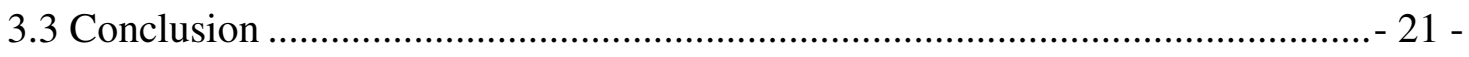

CHAPTER 4: NANOSCALE ITO TOP GRATING ……………………………....... 23 -

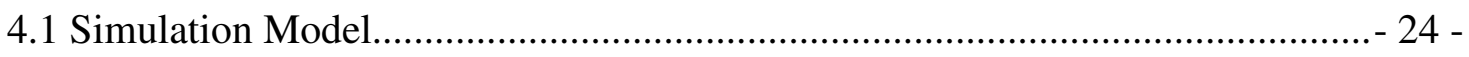

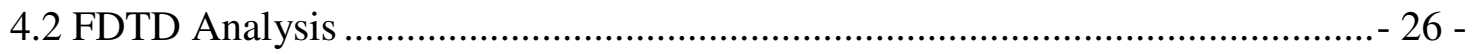

4.2.1 ITO and GaN top grating ……………………….................................. 26 -

4.2.2 Optimizing grating period..................................................................... 28 -

4.2.3 ITO top grating with Ag reflection layer .......................................................- 29 -

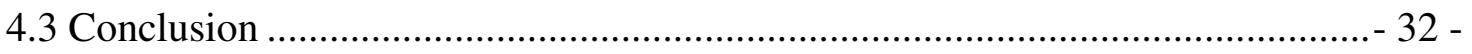

CHAPTER 5: PATTERNED SAPPHIRE SUBSTRATE AND $\mathrm{SIO}_{2}$ ARRAY ...........- 33 - 


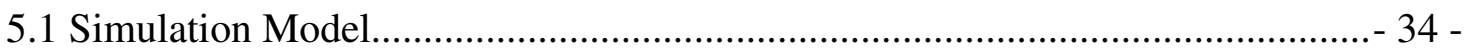

5.2 Matching Previous Studies ............................................................................. 36 -

5.3 Patterned Sapphire Substrate Optimization .......................................................- 38 -

5.4 Optimizing $\mathrm{SiO}_{2}$ Array …………............................................................. 39 -

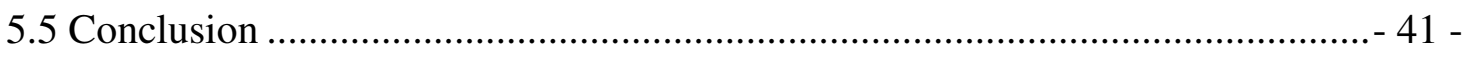

CHAPTER 6: PSS, $\mathrm{SIO}_{2}$ ARRAY AND AG REFLECTION LAYER ........................ 42 -

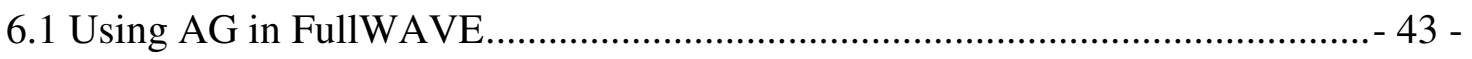

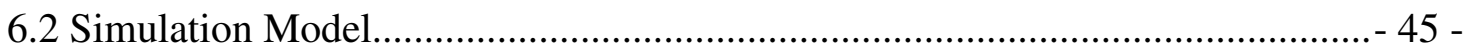

6.2.1 Problems encountered with the software …………………………...........- 47 -

6.2.2 Silver between PSS and n-GaN .............................................................. 50 -

6.3 Optimizing Sapphire Substrate Height ……………….................................... 52 -

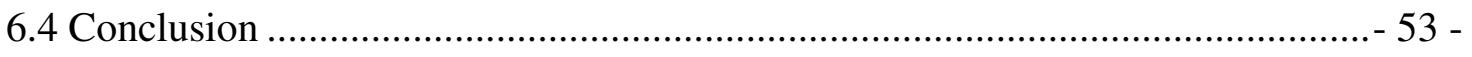

CHAPTER 7: CONCLUSION AND FUTURE WORK …………………………..... 55 -

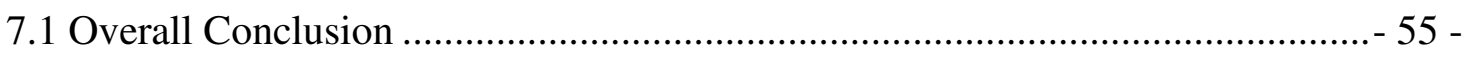

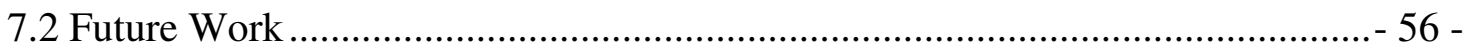

APPENDIX A - FINITE DIFFERENCE TIME DOMAIN METHOD........................ - 58 -

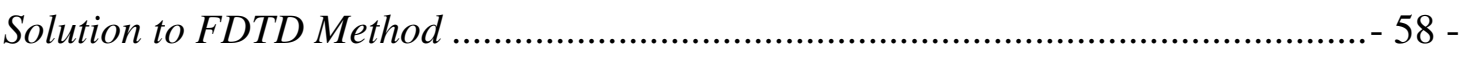

APPENDIX B - FULLWAVE CAD ENVIRONMENT ............................................... 63 -

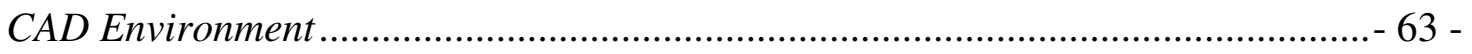

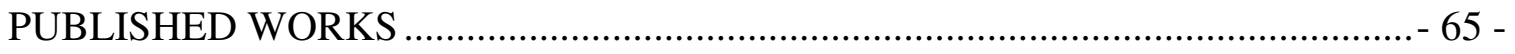

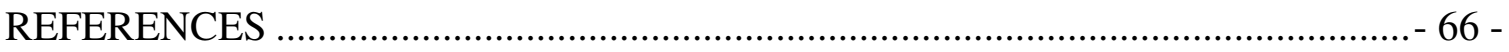




\section{LIST OF TABLES}

Table 3-1 List of LED materials and their respective refractive index and thickness $-16-$

Table 3-2 Summary of maximum light extraction data. $21-$

Table 4-1 LED material thickness and refractive index. $-24-$

Table 4-2 Critical angle based on surrounding medium and grating material. $27-$ Table 4-3 Increased light extraction for LEDs with and without an Ag reflection layer. $-29-$

Table 4-4 Average power extracted for four different structures when sapphire substrate is $10 \mu \mathrm{m}$.

Table 4-5 Average power extracted for four different structures when sapphire substrate is $80 \mu \mathrm{m}$. $-31-$

Table 5-1 Conventional GaN LED Parameters $-35-$

Table 5-2 Simulation setup parameters $-35-$

Table 5-3 Light extraction improvement compared to the conventional LED. $-38-$ Table 5-4 Light extraction improvement by changing PSS width (w) and period (d). $-39-$

Table 5-5 Light extraction improvement by changing $\mathrm{SiO}_{2}$ array position $-40-$

Table 6-1 Simulation parameters used with Ag reflection layer. $-50-$ Table 6-2 Percent improvement of Ag layer compared to LEDs without an Ag layer. $52-$ 


\section{LIST OF FIGURES}

Figure 1.1 Example of a direct band gap emitting a photon...................................... 3 -

Figure 1.2 Example of an indirect band gap emitting a phonon.................................. 3 -

Figure 1.3 Two flow MOCVD diagram. ................................................................ 5 -

Figure 1.4 Total internal reflection at a GaN/Air interface. $\theta_{\mathrm{c}}$ represents the

critical angle and $\theta_{\mathrm{e}}$ represents the emission angle. …............................................. 7 -

Figure 2.1 FullWAVE simulation domain............................................................. 11 -

Figure 2.2 FullWAVE simulation parameters menu. .......................................... 12 -

Figure 3.1 Thin film LED simulation model. ........................................................ 15 -

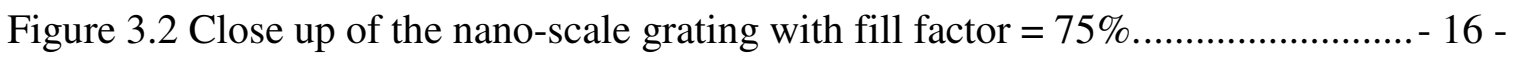

Figure 3.3 Average power plotted versus grating height for cylindrical grating.........- 18 -

Figure 3.4 Average power plotted versus grating height for conical grating. .............. 19 -

Figure 3.5 (a) Cylindrical grating average power for several fill factors; (b)

conical grating average power for several fill factors.......................................... 20 -

Figure 4.1 LED simulation model with ITO based grating. .................................... 24 -

Figure 4.2 Peking University RCWA simulation results. Transmission at normal

direction as a function of grating depth and filling factor. ................................... 25 -

Figure 4.3 Peking University RCWA simulation results. Transmission as a

function of filling factor and grating period. ................................................... 26 -

Figure 4.4 Light extraction of GaN to air, GaN to Epoxy, ITO to air, and ITO to

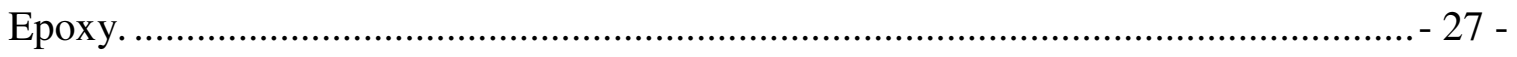

Figure 4.5 Average power vs. grating period for an LED with and without a silver

reflection layer. The grating is ITO and the background is epoxy. 
Figure 4.6 Average power extracted plotted against time when sapphire substrate equals $10 \mu \mathrm{m}$. $-30-$

Figure 4.7 Average power extracted plotted against time when sapphire substrate thickness is $80 \mu \mathrm{m}$.

Figure 4.8 Average power vs. sapphire thickness for four different structures: (1) conventional, (2) conventional with Ag reflection layer, (3) ITO grating, and (4) ITO grating with Ag reflection layer. $-32-$

Figure 5.1 Example of stacking faults blocking threading dislocations adapted from National Chiao-Tung University et al. ${ }^{29}$ $-34-$

Figure 5.2 Labeled diagram of LED with $\mathrm{PSS}$ and $\mathrm{SiO}_{2}$ array. $-36-$ Figure 5.3 Diagram of LED with PSS and $\mathrm{SiO}_{2}$ array. The diagram defines the PSS period (d), width (w), and shows the $\mathrm{x}$ and $\mathrm{z}$ direction of the LED. $-36-$ Figure 5.4 Average power of light extracted in four structures: conventional LED, patterned sapphire substrate, $\mathrm{SiO} 2$ nanorod, and patterned sapphire substrate with $\mathrm{SiO}_{2}$ nanorod PSS width $\mathrm{w}=2.5 \mu \mathrm{m}$ and period $\mathrm{d}=3 \mu \mathrm{m} . \mathrm{SiO}_{2}$ layer is centered at $\mathrm{z}=7.8 \mu \mathrm{m}$

Figure 5.5 Average power as a function of patterned sapphire substrate (PSS) width and PSS period. $-39-$

Figure 5.6 Average power as a function of nanorod array position. In the case with a PSS, $d=3 u m, w=2.5 u m$, and $d=2 u m$ and $w=1.3 u m$. $-40-$

Figure 6.1 Material editor displaying the properties used for Ag. $44-$ Figure 6.2 Real (blue) and imaginary (green) refractive index for Ag. . $-45-$ 
Figure 6.3 LED with Ag reflection layer between sapphire substrate and un-doped $\mathrm{GaN}$

Figure 6.4 LED with Ag reflection layer below the sapphire substrate. The variable $\mathrm{H}$ represents sapphire substrate height. $-46-$ Figure 6.5 Optical field distribution of an LED without Ag. $-47-$ Figure 6.6 Optical field distribution of an LED with an Ag reflection layer. $-48-$ Figure 6.7 Uniform grid size with overlap between $\mathrm{Ag}$ and $\mathrm{GaN}$ interface. $-49-$ Figure 6.8 Realigned grid spacing. $50-$ Figure 6.9 Average power of light extracted in four structures with an Ag reflection layer: conventional $\mathrm{LED}$, patterned sapphire substrate, $\mathrm{SiO} 2 \mathrm{PhC}$, and patterned sapphire substrate with $\mathrm{SiO} 2$ nanorod. PSS width $\mathrm{w}=2.5 \mu \mathrm{m}$ and period $\mathrm{d}=3 \mu \mathrm{m} . \mathrm{SiO}_{2}$ layer is centered at $\mathrm{z}=7.8 \mu \mathrm{m}$. Figure 6.10 Average power vs. sapphire substrate height for four structures with a bottom layer Ag reflector. $53-$ 


\section{CHAPTER 1: INTRODUCTION}

Gallium Nitride (GaN) based Light Emitting Diodes (LED) continue to prove themselves increasingly useful in the world of solid state electronics and lighting. Applications include backlit displays, high end flashlights, household lighting, among many others. Different branches of society push advancement in LED technology with various motivations. Companies that fabricate devices desire to sell a useful product, with good performance and at a maximum profit. On the other hand, a growing awareness of sustainability drives the demand for low power high brightness household and industrial lighting. Several countries, including Brazil, Venezuela and Switzerland have either completely phased out or started to phase out the use of incandescent bulbs due to their inefficiency. ${ }^{1}$ A study performed in 2010 by the Office of Energy Efficiency and Renewable Energy supports that switching to LED lighting over the next twenty years could reduce electricity consumption in lighting by $25 \%$, save the US 120 billion dollars in energy costs, and stop 246 million metric tons of carbon emission. ${ }^{2}$ Such savings are a result of high luminous efficacy, the amount of lumens produced per watt. Typical incandescent bulbs have a luminous efficacy of 14 lumens per watt, while LEDs perform near 100 lumens per watt. Recent laboratory tests by CREE have shown efficacy as high as 254 lumens per watt. ${ }^{3}$ This thesis proposes to increase efficacy by improving light extraction efficiency. To understand the process of improving efficiency, we first explain some semiconductor basics. 


\subsection{Background}

Several factors make GaN a good semiconductor for light emitting applications, the first being its direct band gap property. The Fermi level of a semiconductor lies in between the conduction band and valence band. When temperature equals 0 Kelvin, no electrons occupy energy states above the Fermi level, hence no conduction. As temperature increases, electrons gain energy and jump to the conduction band. Since electrons want to be in the lowest state possible, they often jump back down to the valence band and recombine with holes. As the electrons drop to a lower state, they release energy in the form of light (photons) or heat (phonons). With a direct band gap, momentum does not change and when the electron drops to a lower energy level it releases energy as a photon. With an indirect band gap, a change in energy states results in a change in momentum releasing energy in the form of heat.

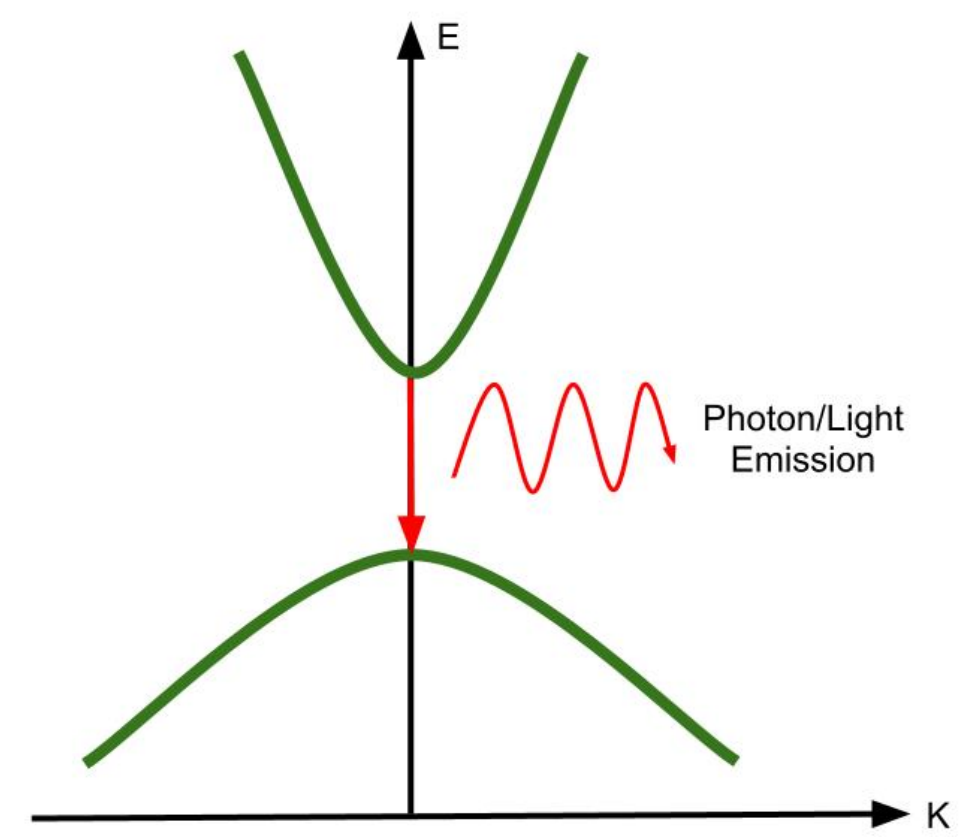

Figure 1.1 and Figure 1.2 are simplified energy band diagrams that display an electron dropping from the conduction band to the valence band emitting either a photon or a phonon. The vertical direction represents electron energy (E), while the horizontal direction $(\mathrm{K})$ represents momentum. 


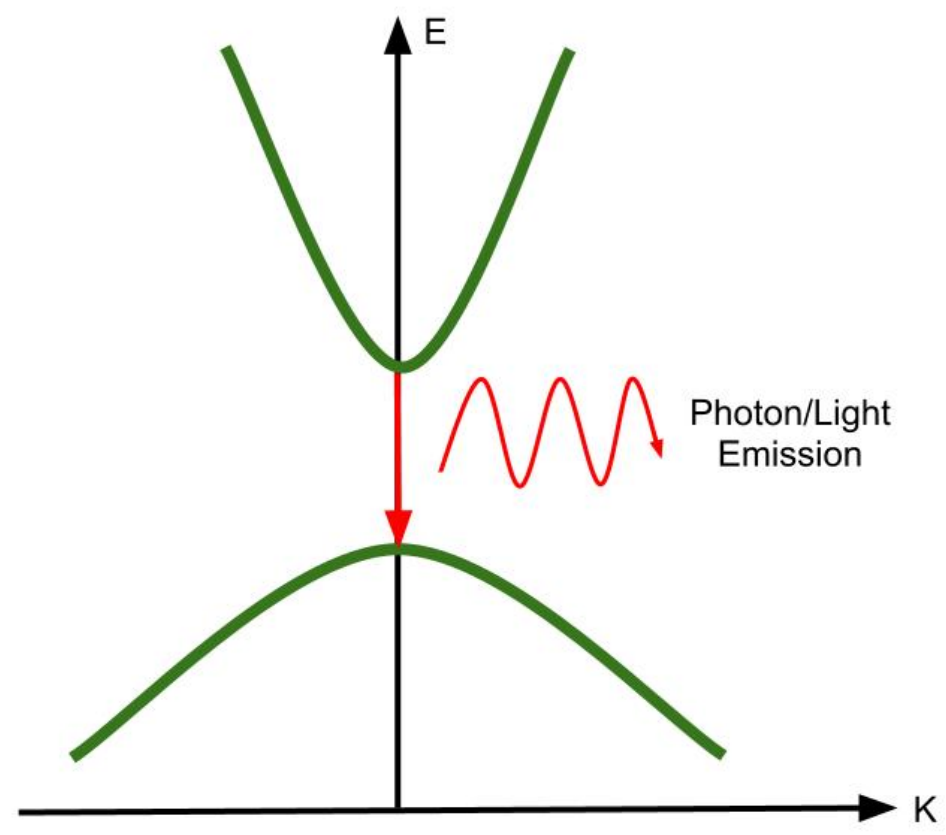

Figure 1.1 Example of a direct band gap emitting a photon.

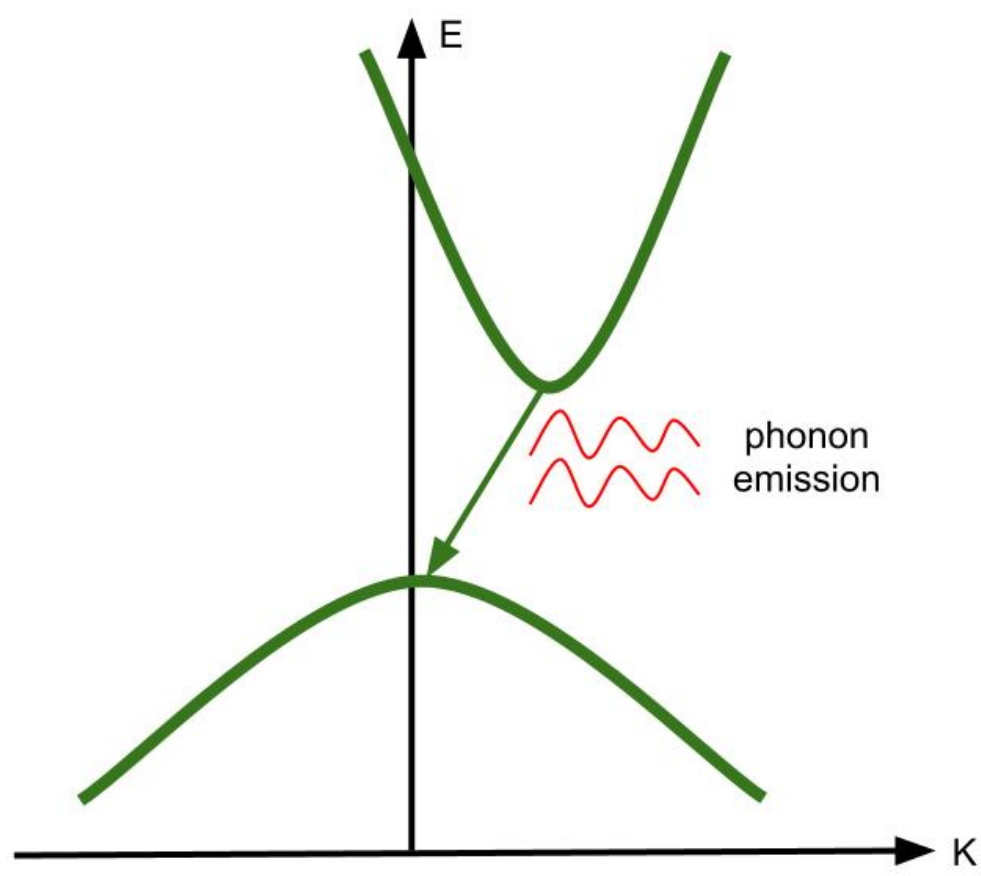

Figure 1.2 Example of an indirect band gap emitting a phonon. 
The wavelength of light emitted from the junction depends on the energy gap between the conduction and valence bands shown by equation 1.1. Different applications require various wavelengths of light.

$\lambda=h c / E_{g}$

The second major advantage of GaN comes from the ability to change the energy gap by mixing in different alloys. For example, adding Indium to $\mathrm{GaN}$ and adjusting the composition of $\operatorname{In}_{\mathrm{x}} \mathrm{Ga}_{1-\mathrm{x}} \mathrm{N}$ varies the band gap from $1.9 \mathrm{eV}$ to $3.4 \mathrm{eV}{ }^{4}$ An energy gap in the range of $1.9 \mathrm{eV}$ to $3.4 \mathrm{eV}$ emits light in the spectrum between yellow and blue. By using different combinations of alloys can range the band gap between $0.7 \mathrm{eV}$ to $6 \mathrm{eV}$ and cover the entire visible spectrum. The variables " $\mathrm{x}$ " and " $1-\mathrm{x}$ " in $\operatorname{In}_{\mathrm{x}} \mathrm{Ga}_{1-\mathrm{x}} \mathrm{N}$ represent alloy percentages. In the above case, when $\mathrm{x}=0.25$ the compound consists of $25 \%$ Indium and $75 \%$ GaN. Changing the composition of these compound semiconductors also changes the refractive index. The new refractive index can be calculated using equation 1.2; the letter $\mathrm{n}$ represents the refractive indices of each material.

$n_{\text {InGaN }}=n_{\text {In }} *(x)+n_{\text {GaN }} *(1-x)$

Conversely, since Indium and Gallium have different lattice constants, increasing the alloy composition increases the lattice mismatch and decreases crystal quality. Above a critical thickness, InGaN can lead to strain related cracking and bowing, limiting device design possibilities. ${ }^{6}$

\subsection{Disadvantages of GaN and Potential Solutions}

Initially, high power GaN based blue LEDs were quite difficult to fabricate due to their poor crystal quality, inability to receive p-type doping, and highly resistive p-type 
layers. In the mid 1990's Shuji Nakamura overcame many of these obstacles and produced the first high power, high luminance GaN based blue LED. ${ }^{7,8}$ Nakamura implemented two techniques to improve crystal quality, reduce resistivity, and dope GaN with acceptors. The first technique, called Two Flow Metal Organic Chemical Vapor Deposition (TFMOCVD), improved the lateral growth of crystals by pumping in gas perpendicular and parallel to the substrate (Figure 1.3). ${ }^{9,10}$

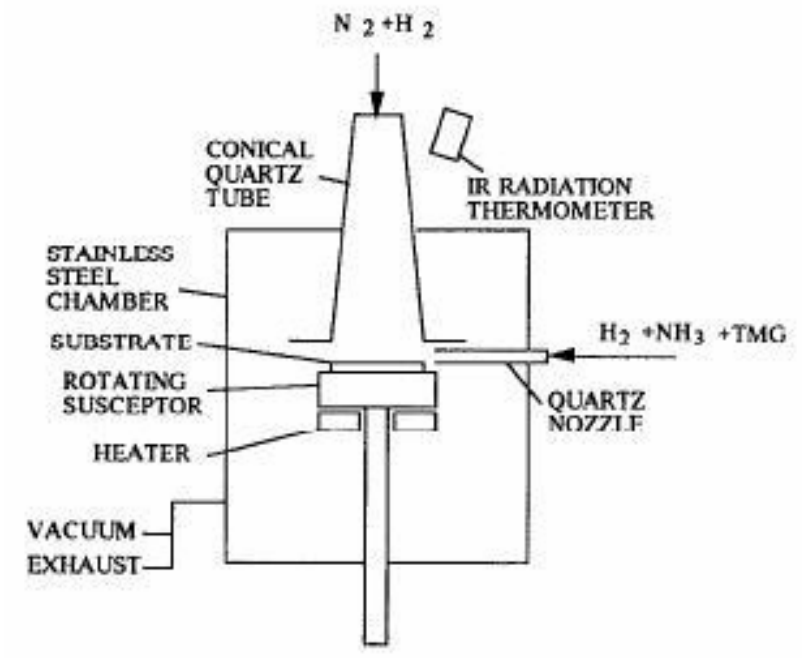

Figure 1.3 Two flow MOCVD diagram.

Second, he developed a process that prevents the p-type semiconductor from becoming intrinsic. This process consisted of annealing the substrate in a hydrogen free environment at high temperatures, releasing p-type impurities bound with hydrogen atoms such as $\mathrm{Mg}-\mathrm{H}$ and $\mathrm{Zn}-\mathrm{H}$.

Today researchers continue to develop creative solutions that increase LED internal quantum efficiency (IQE), the percentage of diode current that produces radiative recombination, and external quantum efficiency (EQE), the ratio of photons that make it into free space to generated photons. ${ }^{11}$ Multiple quantum wells used in LEDs and LDs 
illustrate a technique that improves internal quantum efficiency. Trapping electrons in the well structure improves the recombination efficiency and emits more photons. ${ }^{12}$ External quantum efficiency has three main loss mechanisms: Fresnel loss, photon absorption, and critical angle. Fresnel losses occur due to reflections at dielectric boundary layers. At each interface the incident wave either transmits to the other side or reflects back to be reabsorbed into the semiconductor. Photon absorption occurs most readily in the MQW region since the photon wavelength matches the band gap energy. Absorption introduces two major problems: (1) it reduces EQE and (2) it heats the device via lattice vibrations. Heat changes the device's forward voltage, reduces lifetime, and negatively impacts overall efficiency. Finally, the critical angle determines the angle above which light totally internally reflects between two materials. A large difference in the refractive index of air and $\mathrm{GaN}$ results in a low critical angle and a significant amount of trapped light. Figure 1.4 shows an interface between GaN and Air. The red beam represents light leaving the device. Any light approaching at an angle greater than the critical angle $\left(\theta_{c}\right)$ reflects back into the GaN medium. The critical angle of a device is calculated using Equation 1.3 below where $n_{1}$ and $n_{2}$ represent the refractive index of the two interface materials.

$$
\begin{aligned}
& \theta_{c}=\sin ^{-1}\left(\frac{n_{2}}{n_{1}}\right) \\
& n_{1}>n_{2}
\end{aligned}
$$




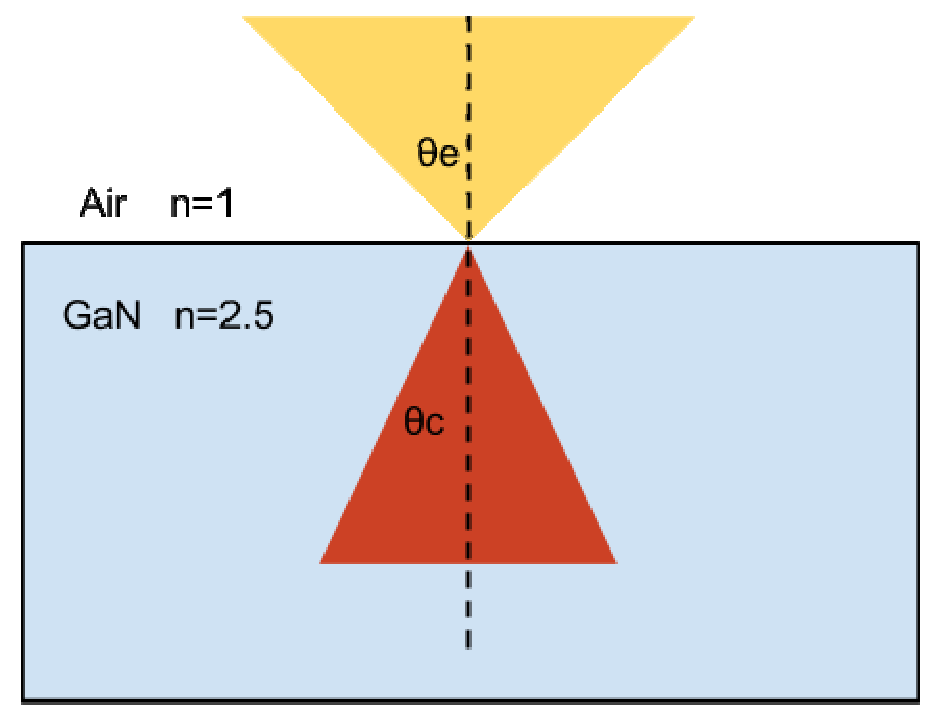

Figure 1.4 Total internal reflection at a GaN/Air interface. $\theta_{c}$ represents the critical angle and $\theta_{\mathrm{e}}$ represents the emission angle.

Fabrication techniques such as periodic top gratings ${ }^{13}$, patterned sapphire substrates ${ }^{14}$, and reflection layers ${ }^{15}$ reduce total internal reflection and improve external quantum efficiency via light extraction enhancement. This study proposes to optimize light extraction efficiency of the above mention LED structures using finite difference time domain analysis (FDTD). Computer simulations give the advantage of analyzing several hundred LED structures in a relatively short time period. This process avoids fabrication time, reduces cost and produces a highly optimized device. The following chapters provide background information on the techniques used and discuss the simulation results. Chapter 2 provides details on the software used. Chapter 3 analyzes how grating period, height, and fill factor affect light extraction. Chapter 4 examines and compares the differences between using an ITO based grating and GaN based grating. Chapter 5 investigates the use of a patterned sapphire substrate and $\mathrm{SiO}_{2}$ nanorod arrays. Chapter 6 
explores Ag-based reflection layers and Chapter 7 summarizes the findings and discusses future work. 


\section{CHAPTER 2: RSOFT FULLWAVE ANALYSIS}

Methods such as Rigorously Coupled Wave Analysis (RCWA), ray tracing, and the Finite Difference Time Domain (FDTD) solution to Maxwell's curl equations exist to simulate light extraction. All simulations in this thesis are performed using Rsoft's FullWAVE software. This module employs FDTD analysis and requires the user to discretize time and space by splitting the physical domain into a grid. Within each grid location, the software calculates the electric field followed by the magnetic field in a "leapfrog" like fashion until reaching steady state. The following sections provide basic information on the software and its use. For a more in depth explanation of the FDTD method and its solution to Maxwell's curl equations, refer to Appendix A.

\subsection{Defining Materials and Grid Size}

Properly simulating a device in FULLWave requires material information and grid size parameters. The material properties are set differently for linearly dispersive materials and nonlinearly dispersive materials. Material dispersion describes how wave velocity varies with wavelength. ${ }^{16}$ For linearly dispersive materials, the parameters are set by entering the refractive index in segment properties. Non-linearly dispersive materials must be defined in the material editor, which uses the sum of Lorentzian functions to describe permittivity as a function of wavelength shown by Equation 2.1.

$\varepsilon(\omega)=\varepsilon_{\infty}+\sum_{k} \frac{\Delta \varepsilon_{k}}{a_{k}\left(i \omega^{2}\right)-b_{k}(i \omega)+c_{k}}$

3D models require mesh size definition in the $\mathrm{x}, \mathrm{y}$, and $\mathrm{z}$ directions. Since 3-D

simulations demands significant time and memory, we perform all simulations in 2-D and only define the $\Delta \mathrm{x}$ grid and $\Delta \mathrm{z}$ grid. The grid size must be small enough to resolve the 
smallest features of the simulation, often the wavelength of light. Structures that contain periodic gratings in the nanometer range sometimes require spatial dimensions smaller than the wavelength. The grid size should be at least smaller than one tenth the wavelength of light in the material or the smallest dimension in the simulation profile. Since FDTD also descretizes the time domain the user must provide a time step $\Delta \mathrm{t}$. The time step is also limited by the dimensions of the grid as described by the Courant condition in Equation 2.2 where $\mathrm{c}$ represents the speed of light, $\Delta \mathrm{t}$ is the time step, and $\Delta \mathrm{x}$, $\Delta y$, and $\Delta \mathrm{z}$ are the spatial dimensions of the grid. According to this equation, as the spatial dimensions decrease, the time step must decrease.

$$
c \Delta t<\frac{1}{\sqrt{\frac{1}{\Delta x^{2}}+\frac{1}{\Delta y^{2}}+\frac{1}{\Delta z^{2}}}}
$$

The FullWAVE simulation software automatically enforces the Courant condition by displaying the Stability Limit in the Time Grid. This shows the minimum required time step based on other simulation parameters. The user is strongly encouraged to refine both the grid size and time step to optimize the simulation. 


\subsection{Simulation Domain}

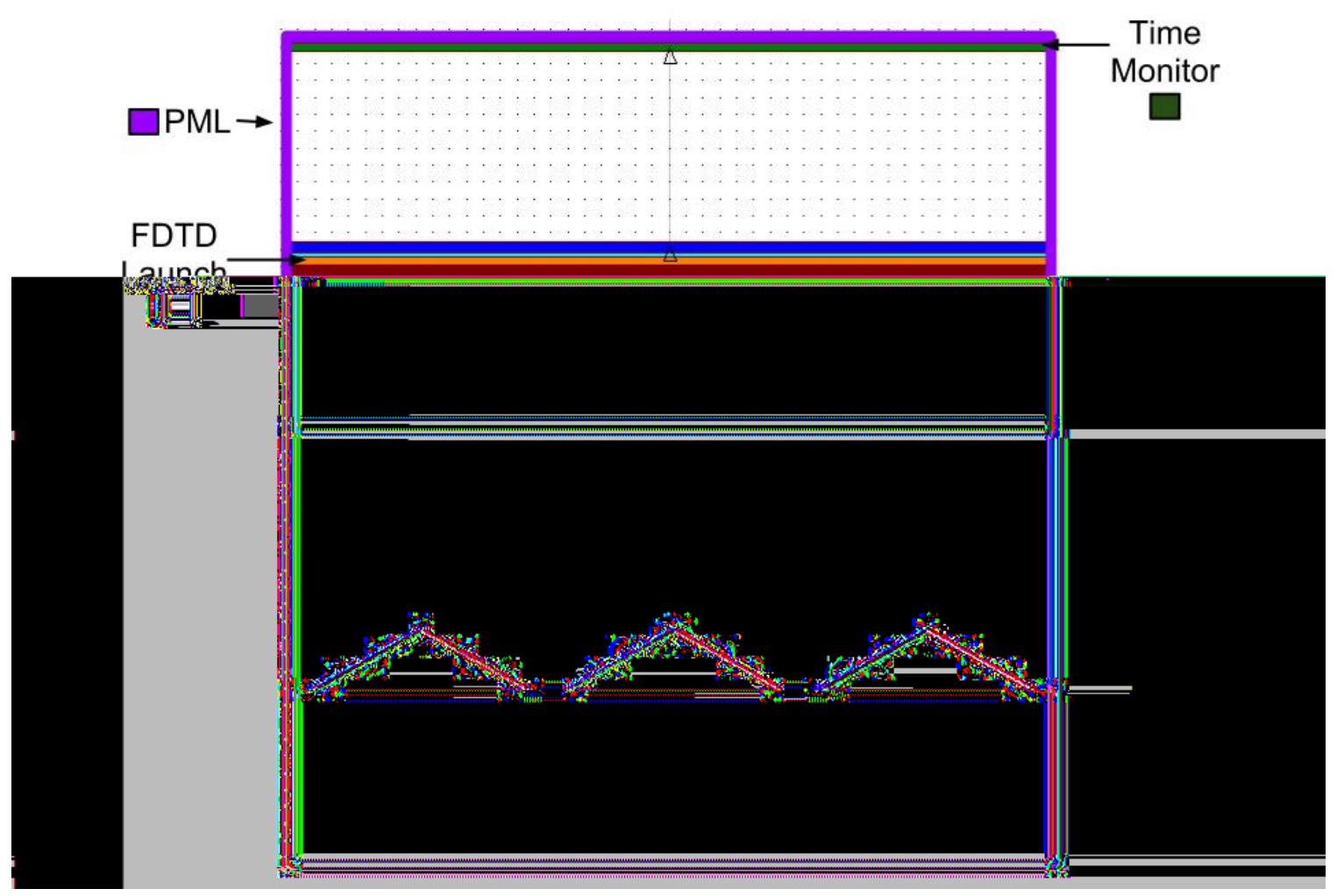

Figure 2.1 FullWAVE simulation domain.

Figure 2.1 illustrates an example of a FullWAVE simulation domain containing a Perfectly Matched Layer (PML), FDTD Launch, Time Monitor, and the LED Structure being simulated. The simulation domain is the area enclosed by the Perfectly Matched Layer (PML) shown in purple. The PML, allows waves to exit the simulation domain without producing unwanted reflections. Clicking the traffic light shaped button shown in Figure B.1 brings up the FullWAVE Simulation Parameters menu shown in Figure 2.2. In this menu, the user defines grid size, time step, PML width and all other FDTD parameters. 


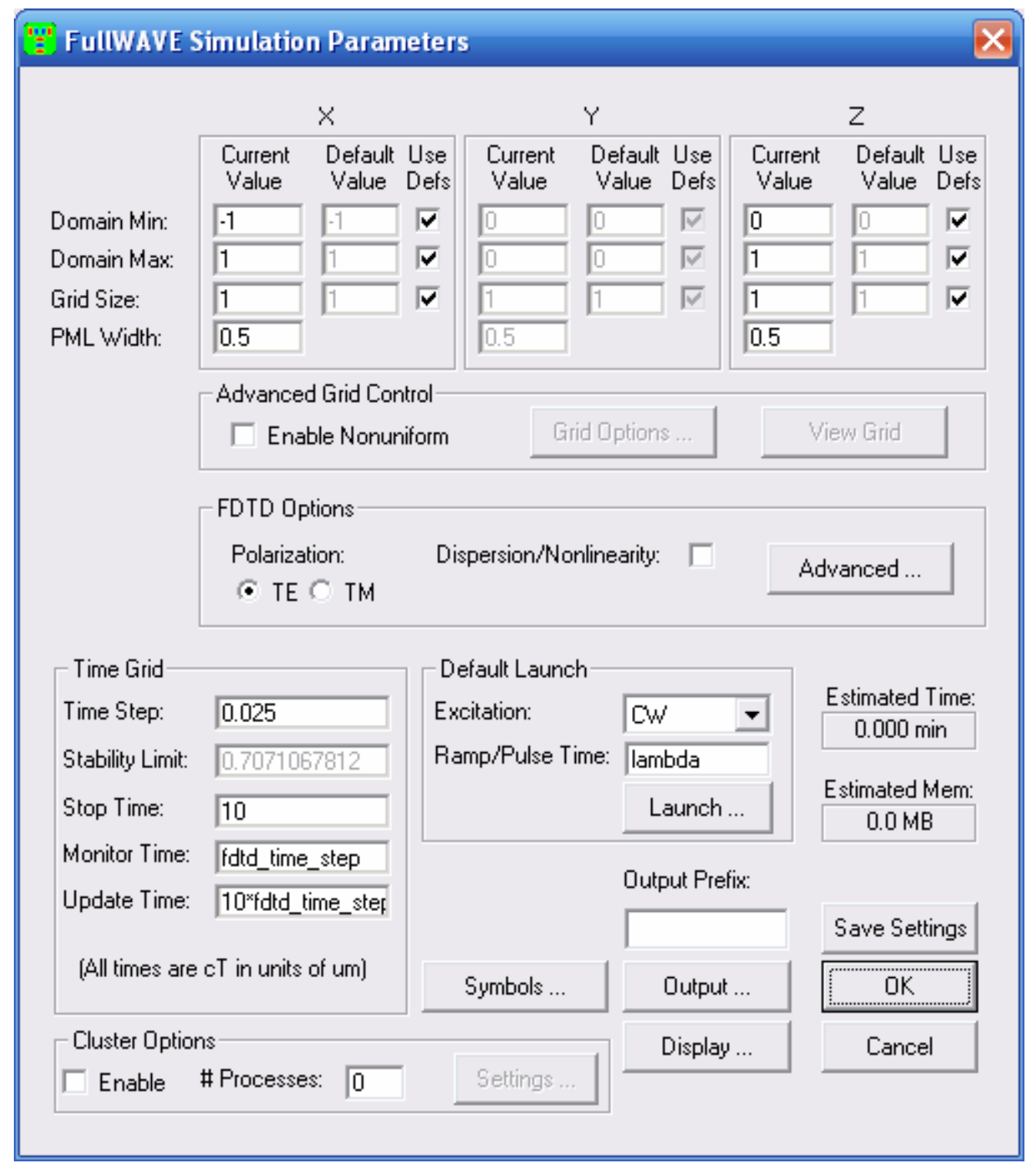

Figure 2.2 FullWAVE simulation parameters menu.

The time monitor displayed in green measures electric field, magnetic field, and

power and produces either a temporal or spatial output. The following simulations use the time monitor to measure average power as a function of time. The output file, stored in the .TMN format, saves the square root of the average power along with its corresponding time component. The file can be opened with programs such as Microsoft 
Excel or KGraph. Typically one time monitor is used to measure light extracted from the top of the LED; however, the use of multiple time monitors on the sides and bottom of an LED provides more information and a clearer picture of the light extraction process. Since time did not permit the use of multiple time monitors in the following simulations, this topic is further discussed in the future work section.

Finally, the FDTD Launch located in the MQW region is the excitation source. The software allows both field and current sources. In all simulations we use a field source which can be thought of as an incident beam radiating from a specified plane. The wavelength of light is chosen in the Launch Parameters and in the Global Settings. In both cases we use a wavelength of $460 \mathrm{~nm}$. This wavelength corresponds to blue, an important color used to fabricate white LEDs. The Launch Parameters also allow the user to choose the type of light emitted. To stay consistent with previous studies and simulations, we use slab mode as the launch type. For more information on how to use FullWAVE's CAD environment refer to Appendix B. 


\section{CHAPTER 3: NANOSCALE TOP TRANSMISSION GRATING}

We begin our simulations by analyzing a thin film GaN based LED with a nanoscale top transmission grating, also described as a photonic crystal grating $(\mathrm{PhC})$. The large difference in refractive index between GaN and air reduces device efficiency by total internal reflection. ${ }^{17}$ Photonic crystal $(\mathrm{PhC})$ gratings provide more angles for light to escape the LED and thus improve extraction efficiency. Different patterns and shapes of photonic crystal gratings can be formed by chemical wet etching, dry etching, and focused ion beam milling (FIBM) ${ }^{18}$ Two specific methods include: electron-beam

lithography and nano-imprint lithography. ${ }^{19}$ Previous studies using simulations ${ }^{20,21,22}$ and experimental data ${ }^{23}$ show that the period, shape, and height of the grating all affect light extraction efficiency. In this first set of simulations we adjust all three of these parameters and observe light extraction enhancement.

\subsection{Simulation model}

Figure 3.1 displays an example of the thin-film LED simulated. The structure consists of a 200nm n-GaN 2PhC grating, 400nm n-GaN substrate, 50nm MQW, and 200nm p-GaN substrate.

Figure 3.1 includes three essential FDTD simulation components: the FDTD launch, time monitor, and perfectly matched layer (PML). The launch serves as a source of light within the simulation, therefore we place it in the center of the multiple quantum wells (MQW) where most electron hole recombination occurs. We choose the light source to be constant wave $(\mathrm{CW})$ at $460 \mathrm{~nm}$, assuming that the LED emits blue light. The light travels 
through the device until it reaches the time monitor. The time monitor takes a time average of power throughout the simulation and stores it in a separate data file. The PML surrounds the simulation window and allows waves to exit without producing any unwanted reflections. Generally, the PML should be ten or more grid points thick in each direction $(\mathrm{x}, \mathrm{y}, \mathrm{z})$, or in this case $(\mathrm{x}, \mathrm{y})$.

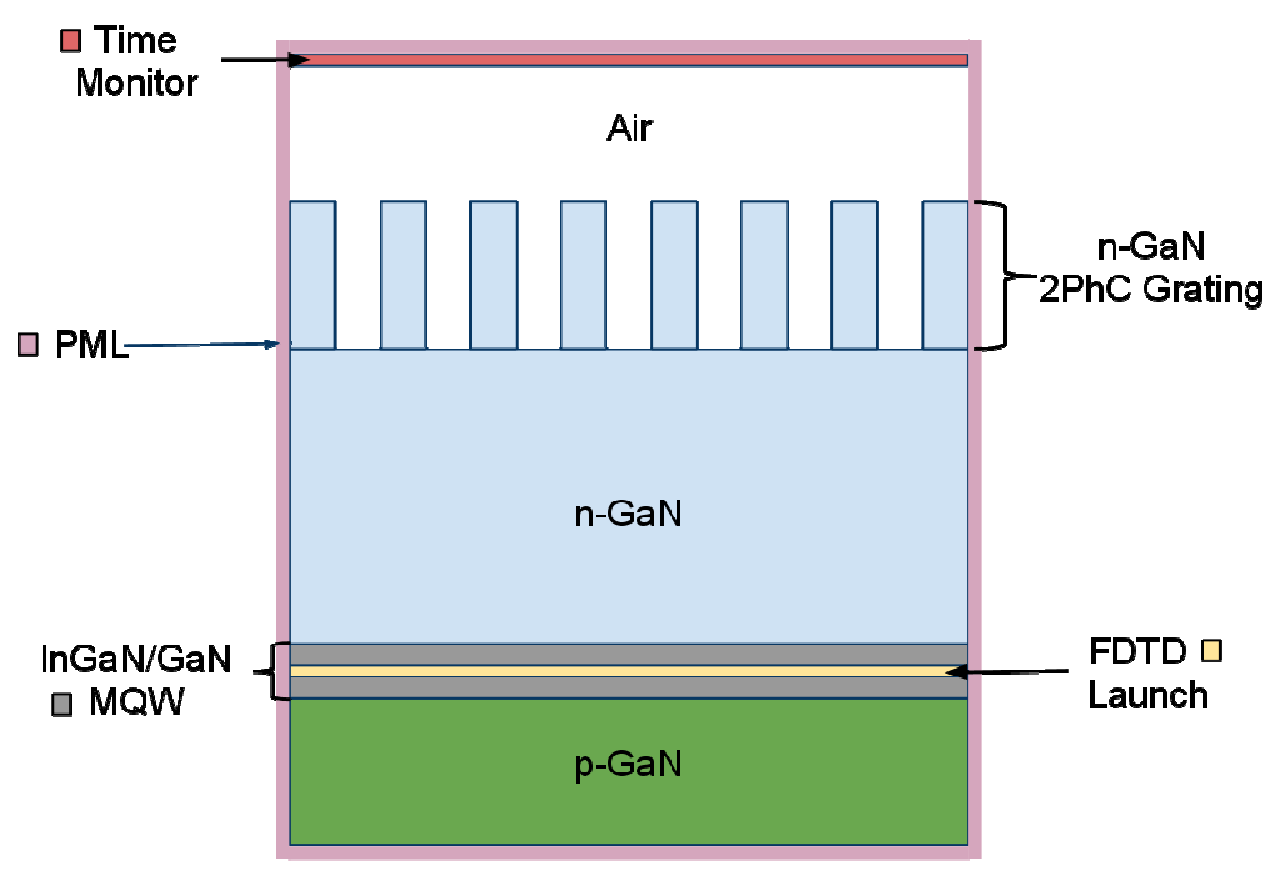

Figure 3.1 Thin film LED simulation model.

Table 3-1 lists the refractive index and thickness of the materials used in this simulation. We focus on optimizing three design parameters: grating height (d), grating period (A), and fill factor (FF). Grating period is the distance between the centers of each grating cell. The fill factor $(\mathrm{FF})$ represents the percentage of $\mathrm{GaN}$ within one period. Figure 3.2 shows a closeup of the grating structure with $\mathrm{FF}=75 \%$. In one period $75 \%$ of the grating is filled with $\mathrm{GaN}$ and $25 \%$ is filled by air. We use a refractive index of 2.5 for all $\mathrm{n}$ and $\mathrm{p}$ type $\mathrm{GaN}$ substrates except the $\mathrm{InGaN} / \mathrm{GaN}$ MQW which has an index of 
refraction equal to 2.6 It is also important to notice that each material has a constant thickness except the $2 \mathrm{PhC}$ grating structure which varies from 50 to $200 \mathrm{~nm}$ in steps of $50 \mathrm{~nm}$.

Table 3-1 List of LED materials and their respective refractive index and thickness.

\begin{tabular}{|c|c|c|}
\hline Material & Refractive Index & Thickness (nm) \\
\hline Air & 1 & N/A \\
\hline $\begin{array}{c}\text { n-GaN 2PhC } \\
\text { Grating }\end{array}$ & 2.5 & $50-200$ \\
\hline n-GaN & 2.5 & 400 \\
\hline $\begin{array}{c}\text { InGaN/GaN } \\
\text { MQW }\end{array}$ & 2.6 & 50 \\
\hline p-GaN & 2.5 & 200 \\
\hline
\end{tabular}

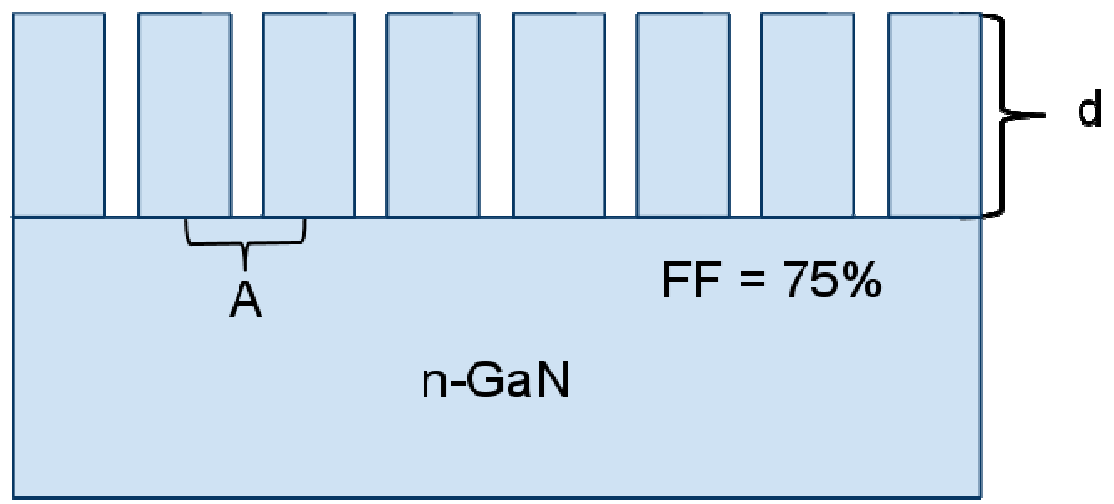

Figure 3.2 Close up of the nano-scale grating with fill factor $=75 \%$.

\subsection{Simulation Results}

\subsubsection{Cylindrical and conical gratings}

In order to optimize light extraction, we compare average power data from three basic models: the first has no grating, the second has a cylindrical shaped grating, and the third 
has a conical shaped grating. Since we simulated in 2-D, the cylindrical grating appears rectangular and the conical grating appears triangular. The first case, with no grating, serves as a base value to compare with other results. Any improvements in light extraction are weighed against the results from this model. Since the grating period (A) and fill factor (FF) cannot be changed for this model, we change the height (d) from 50 to $200 \mathrm{~nm}$ in steps of $50 \mathrm{~nm}$. The total $\mathrm{n}-\mathrm{GaN}$ substrate height changes from $600 \mathrm{~nm}$ to $450 \mathrm{~nm}$.

Next, for the cylindrical and conical models, we set the fill factor to $50 \%$ and sweep the grating period from $100 \mathrm{~nm}$ to $1000 \mathrm{~nm}$ in steps of $100 \mathrm{~nm}$. The grating height increases from $50 \mathrm{~nm}$ to $200 \mathrm{~nm}$ in steps of $50 \mathrm{~nm}$ for each period. The results for the cylindrical model (Figure 3.3) show that light extraction improves only for a grating period of $A=100 \mathrm{~nm}$. Unexpectedly, in all other cases the grating reduces light extraction compared to the non-grating model. In the case of the cylindrical model, maximum average power occurs when the grating period $A=100 \mathrm{~nm}$ and grating height $\mathrm{d}=50 \mathrm{~nm}$. In this case, light extraction increases by $10.8 \%$ compared to the non-grating model with $\mathrm{d}=200 \mathrm{~nm}$. The general trend shows that as grating period increases, light extraction decreases. Light extraction begins to improve above $A=400 \mathrm{~nm}$, although it still performs below the non-grating model. The trend for grating height effects is not as well defined as the grating period. In some cases, such as A $=300 \mathrm{~nm}$, a larger grating height improves efficiency and in other cases, such as A $=100 \mathrm{~nm}$, the smallest grating height improves efficiency. However, for grating periods A > 300nm an increase in grating height generally decreases average power. 

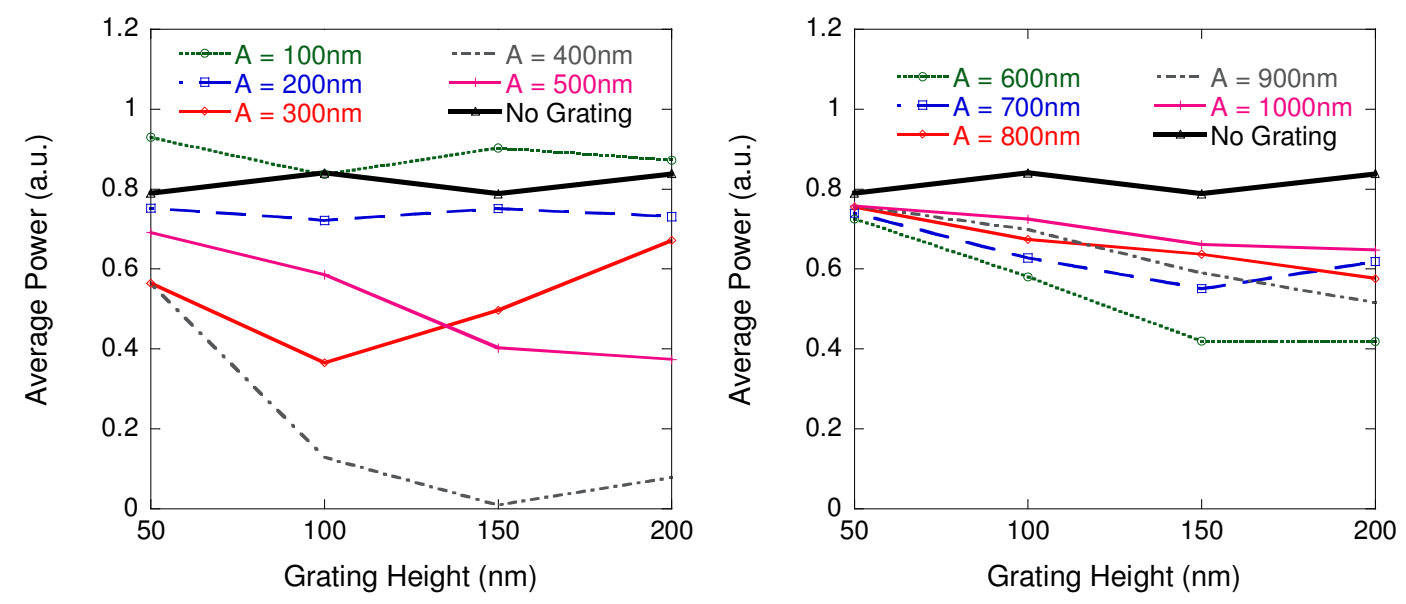

Figure 3.3 Average power plotted versus grating height for cylindrical grating.

The results for the conical grating model (Figure 3.4), show that light extraction improves for grating period $\mathrm{A}=100 \mathrm{~nm}$ and in some cases $\mathrm{A}=200 \mathrm{~nm}$. All other grating periods result in decreased light extraction relative to the non-grating model. Unlike the cylindrical model, maximum average power occurs when $A=100 \mathrm{~nm}$ and $d=100 \mathrm{~nm}$. In this case light extraction increases by $16.8 \%$ relative to the non-grating model with height $\mathrm{d}=200 \mathrm{~nm}$. Relative to the cylindrical model maximum, the conical model increases light extraction by $5.4 \%$. The general trend shows similarities to the cylindrical model. An increase in grating period results in a decrease in light extraction. When A > 400nm light extraction begins to improve, but never reaches non-grating performance. For cases where $\mathrm{A}>300 \mathrm{~nm}$, an increase in grating height seems to decrease overall performance. 

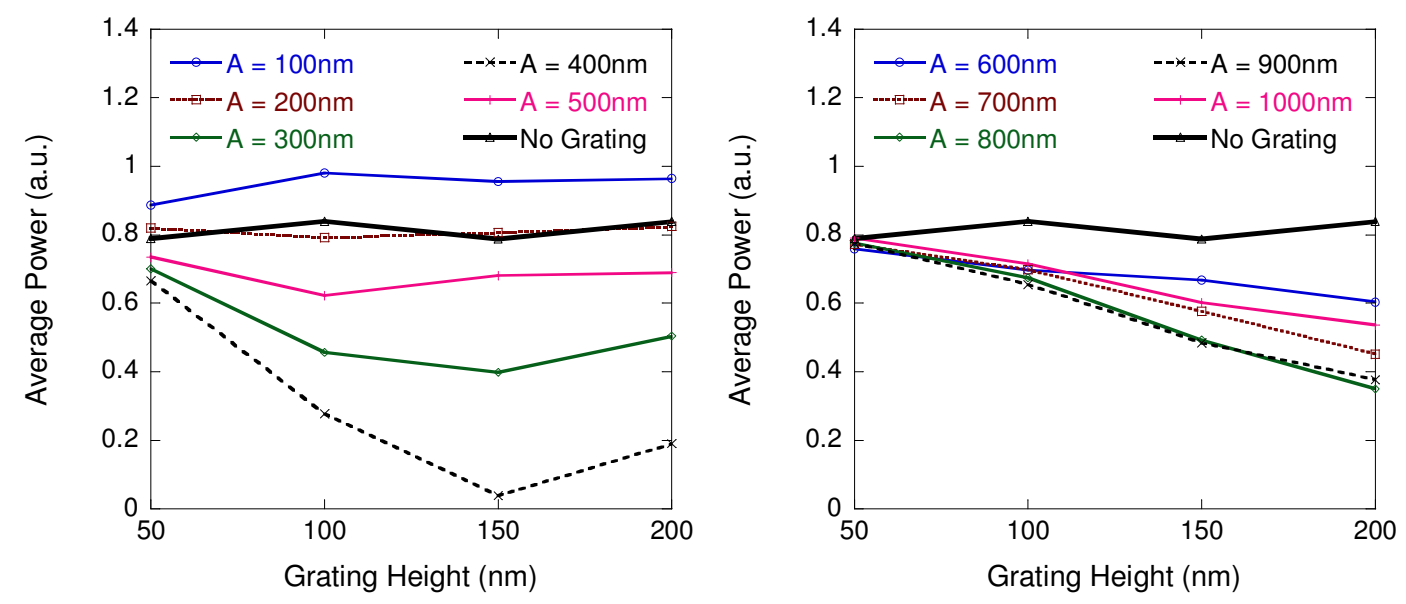

Figure 3.4 Average power plotted versus grating height for conical grating.

\subsubsection{Changing the fill factor}

The final simulations set the grating period to $100 \mathrm{~nm}$ and alter both the grating height and fill factor. We maintain a grating period of $100 \mathrm{~nm}$ because the most improvement in both devices occurs at this period as seen in Figure 3.3 and Figure 3.4. We alter the grating height from 50nm to $200 \mathrm{~nm}$ in steps of $50 \mathrm{~nm}$ and change the fill factor to the following five values: $25 \%, 33 \%, 50 \%, 66 \%$, and $75 \%$. The results for the cylindrical grating in Figure 3.5 show a $17.8 \%$ increase in light extraction for grating height $\mathrm{d}=$ $200 \mathrm{~nm}$ and $\mathrm{FF}=33 \%$ compared to the non-grating case when $\mathrm{d}=200 \mathrm{~nm}$. It also shows a $6.3 \%$ increase compared to the previous cylindrical maximum value. The results for the conical grating in Figure 3.5 show a $17.8 \%$ increase in average power for grating height d $=200 \mathrm{~nm}$ and $\mathrm{FF}=66 \%$ relative to the non-grating case where $\mathrm{d}=200 \mathrm{~nm}$. In general, increasing and decreasing the fill factor from $50 \%$ results in either an increase in light extraction or decrease depending on the grating height and shape. 


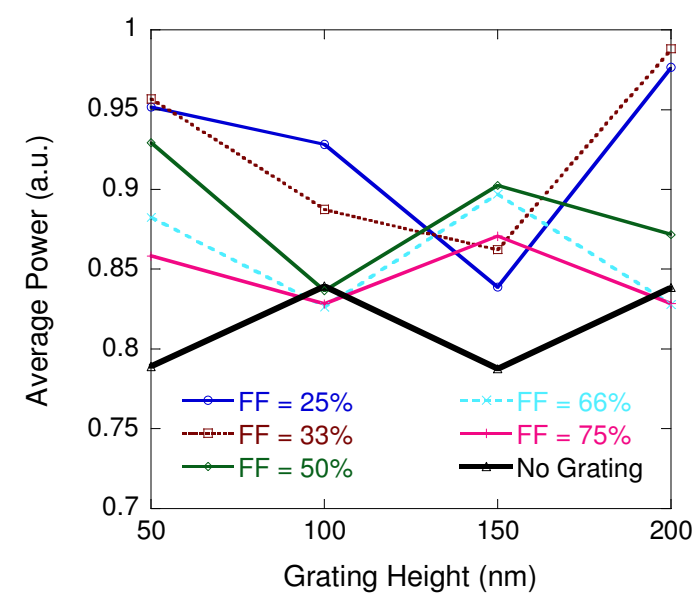

(a)

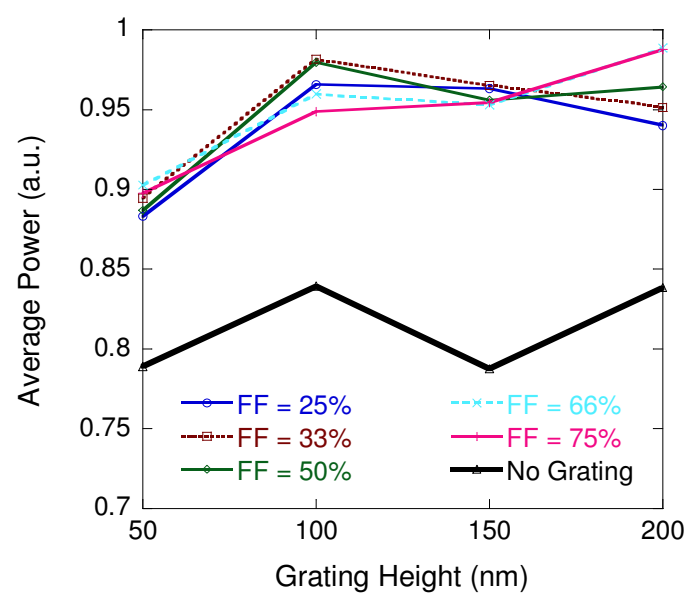

(b)

Figure 3.5 (a) Cylindrical grating average power for several fill factors; (b) conical grating average power for several fill factors.

Table 3-2 serves to clarify and summarize the results of this study. It lists the grating type, grating period (A), grating height (d), fill factor (FF), average power, and the increase in light extraction relative to the non-grating case where $d=200 \mathrm{~nm}$. The average power column illustrates that different non-grating cases have different light extraction efficiencies; we used the $\mathrm{d}=200 \mathrm{~nm}$ case to compare all other data against. The table also highlights the two structures with the highest light extraction by using boldface font. 
Table 3-2 Summary of maximum light extraction data.

\begin{tabular}{|c|c|c|c|c|c|}
\hline $\begin{array}{l}\text { Grating } \\
\text { Type }\end{array}$ & $\mathrm{A}(\mathrm{nm})$ & $\mathrm{d}(\mathrm{nm})$ & $\mathrm{FF}(\%)$ & $\begin{array}{c}\text { Average } \\
\text { Power } \\
\text { (a.u.) }\end{array}$ & $\begin{array}{c}\text { Relative } \\
\text { Increase } \\
(\%)\end{array}$ \\
\hline \multirow[t]{2}{*}{ Cylindrical } & 100 & 50 & 50 & 0.929 & 10.8 \\
\hline & 100 & 200 & 33 & 0.988 & 17.8 \\
\hline \multirow[t]{2}{*}{ Conical } & 100 & 100 & 50 & 0.979 & 16.8 \\
\hline & 100 & 200 & 66 & 0.988 & 17.8 \\
\hline \multirow[t]{4}{*}{$\begin{array}{c}\text { Non- } \\
\text { Grating }\end{array}$} & $\mathrm{n} / \mathrm{a}$ & 50 & $\mathrm{n} / \mathrm{a}$ & 0.78913 & 0 \\
\hline & $\mathrm{n} / \mathrm{a}$ & 100 & $\mathrm{n} / \mathrm{a}$ & 0.83955 & 0 \\
\hline & $\mathrm{n} / \mathrm{a}$ & 150 & $\mathrm{n} / \mathrm{a}$ & 0.78778 & 0 \\
\hline & $\mathrm{n} / \mathrm{a}$ & 200 & $\mathrm{n} / \mathrm{a}$ & 0.83856 & 0 \\
\hline
\end{tabular}

\subsection{Conclusion}

FDTD analysis shows that a nano-structure top grating does improve light extraction efficiency only for small grating period. Most grating periods larger than 100nm resulted in lower efficiency. In the case where fill factor is set to $50 \%$, the conical grating showed a $16.7 \%$ increase in average power compared to the non-grating model and a $5.4 \%$ compared to the cylindrical model. Changing the fill factor showed that, in general the conical model outperforms the cylindrical model. However, the clyindrical and conical models obtain the same maximum average power. The cylindrical model's maximum average power occurred for $\mathrm{A}=100 \mathrm{~nm}, \mathrm{~d}=200 \mathrm{~nm}$, and $\mathrm{FF}=33 \%$. The conical model's maximum average power occurred for $A=100 \mathrm{~nm}, \mathrm{~d}=200 \mathrm{~nm}$, and $\mathrm{FF}=66 \%$. These results provide an outline to design thin film GaN based LED's with a nano-structure grating.

Although interesting, these results do not match the simulations and experimental data shown by Kyungpook National University and Peking University. Kyungpook National University also employed a 2-D FDTD model to observe transmission 
efficiency as a function of grating period and grating depth. They varied the grating period from $0.5 \lambda$ to $2.5 \lambda$ and the grating height from $0.5 \lambda$ to $3 \lambda$. Their results show that changing the grating period has little to no effect in transmission efficiency, while increasing the grating height improves transmission efficiency. ${ }^{24}$ Peking University fabricated $\mathrm{GaN}$ based LEDs with $2 \mathrm{PhC}$ arrays. The lattice constant, or grating period, varies from $230 \mathrm{~nm}$ to $1500 \mathrm{~nm}$ ( $0.5 \lambda$ to $3.2 \lambda)$. Using both a microscopic electroluminescence system and RCWA simulations they show that maximum light extraction occurs for grating periods $230 \mathrm{~nm}$ and $460 \mathrm{~nm} .{ }^{25}$ All three studies show conflicting results. Our study claims maximum light extraction with a grating period of 100nm, Peking University claims maximum extraction with a period of $460 \mathrm{~nm}$, and Kyungpook University claims that grating periods on a wavelength scale do not affect light extraction. In the following chapter we resimulate this structure and compare it with an Indium Tin Oxide (ITO) based top grating LED. 


\section{CHAPTER 4: NANOSCALE ITO TOP GRATING}

Today's advanced technology allows engineers to fabricate LEDs with various heights, widths, shapes, and materials. Materials affect LED forward voltage, emitted wavelength, and light extraction efficiency. Total internal reflection, previously discussed in Chapter 1, prevents light from escaping the LED. The ratio of refractive indices at the boundary, determine the critical angle, or minimum angle at which light completely reflects. A large difference in refractive indices results in a small critical angle and more reflected light. A small difference in refractive indices results in a large critical angle, less light reflected back and more light transmitted into the following medium. Changing the material of the top grating from GaN to ITO increases the critical angle from 23.5 degrees to 28.4 degrees. Additionally, surrounding the chip with an epoxy dome further increases the critical angle to 47.9 degrees. For these reasons, this chapter analyzes light extraction enhancement of an ITO to epoxy interface. Section 4.1 illustrates and describes the simulation model and discusses results and limitations of the RCWA simulations provided by the Peking University Physics Department. Section 4.2 uses FDTD analysis to compare light extraction efficiency for ITO and GaN gratings, optimize ITO grating period, and investigate the effects of an Ag reflection layer. Section 4.3 concludes this chapter by summarizing the results. 


\subsection{Simulation Model}

EPOXY

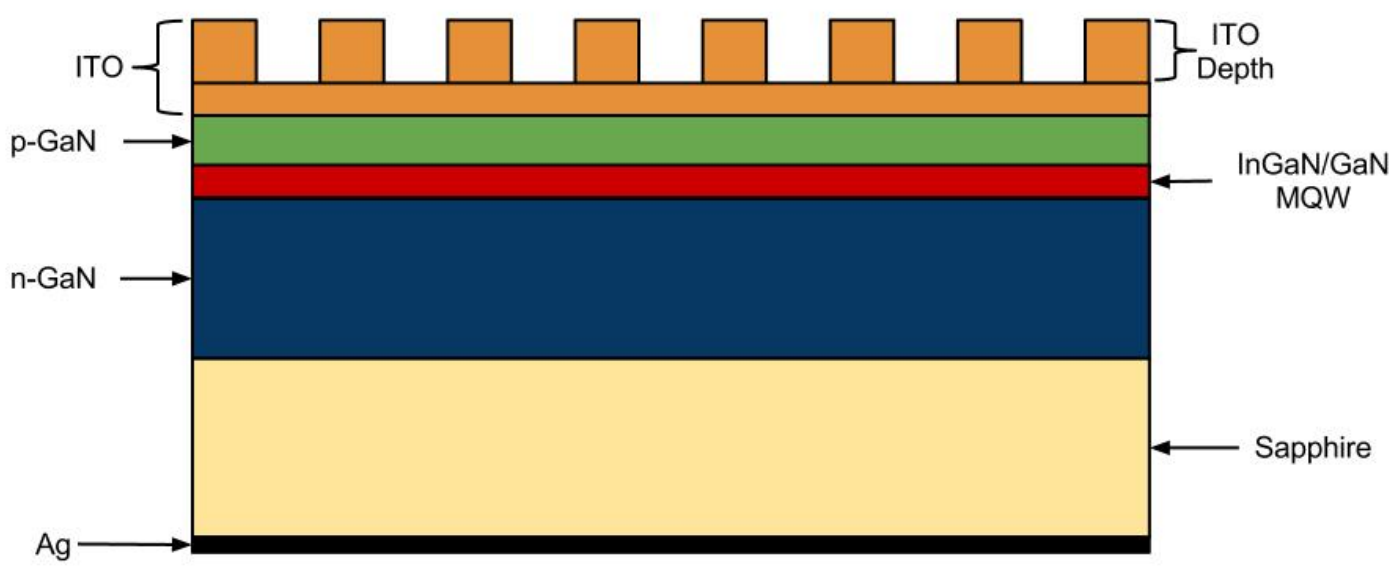

Figure 4.1 LED simulation model with ITO based grating.

Table 4-1 LED material thickness and refractive index.

\begin{tabular}{|c|c|c|}
\hline Material & $\begin{array}{c}\text { Thickness } \\
(\mu \mathrm{m})\end{array}$ & $\begin{array}{c}\text { Refractive } \\
\text { Index }\end{array}$ \\
\hline $\mathrm{p}-\mathrm{GaN}$ & 0.2 & 2.5 \\
\hline $\begin{array}{c}\text { InGaN/GaN } \\
\text { MQWs }\end{array}$ & 0.1 & 2.6 \\
\hline $\mathrm{n}-\mathrm{GaN}$ & 2 & 2.5 \\
\hline $\mathrm{GaN}$ & 3 & 2.5 \\
\hline Sapphire & 80 & 1.78 \\
\hline ITO & 0.23 & 2.1 \\
\hline Epoxy & $>10000$ & 1.56 \\
\hline $\mathrm{Ag}$ & \multicolumn{2}{|c}{ Reflection: $90 \%$} \\
\hline \multicolumn{3}{|l}{}
\end{tabular}

The LED model shown in Figure 4.1 consists of an ITO top grating, p-GaN layer,

InGaN/GaN MQW, n-GaN layer, sapphire substrate, and an Ag reflection layer. Table 4-1 includes the material thickness and refractive index of each layer. As part of a collaborative effort, students at Peking University used Rigorously Coupled Wave Analysis (RCWA) to analyze perpendicular light transmitted as a function of filling factor, grating depth, and grating period. Figure 4.2 shows light transmission as a function of both grating depth and filling factor. The dark orange spots represent high 
transmission, while the blue and green areas represent lower transmission levels. The results reveal an optimal grating depth of $100 \mathrm{~nm}$ and fill factor of 0.5. Similarly, Figure 4.3 graphs transmission as a function of fill factor and grating period. From this graph we obtain an optimal grating period of $1130 \mathrm{~nm}$ with a fill factor of 0.5 . To validate Peking University's results, we simulate the same structure using FDTD analysis.

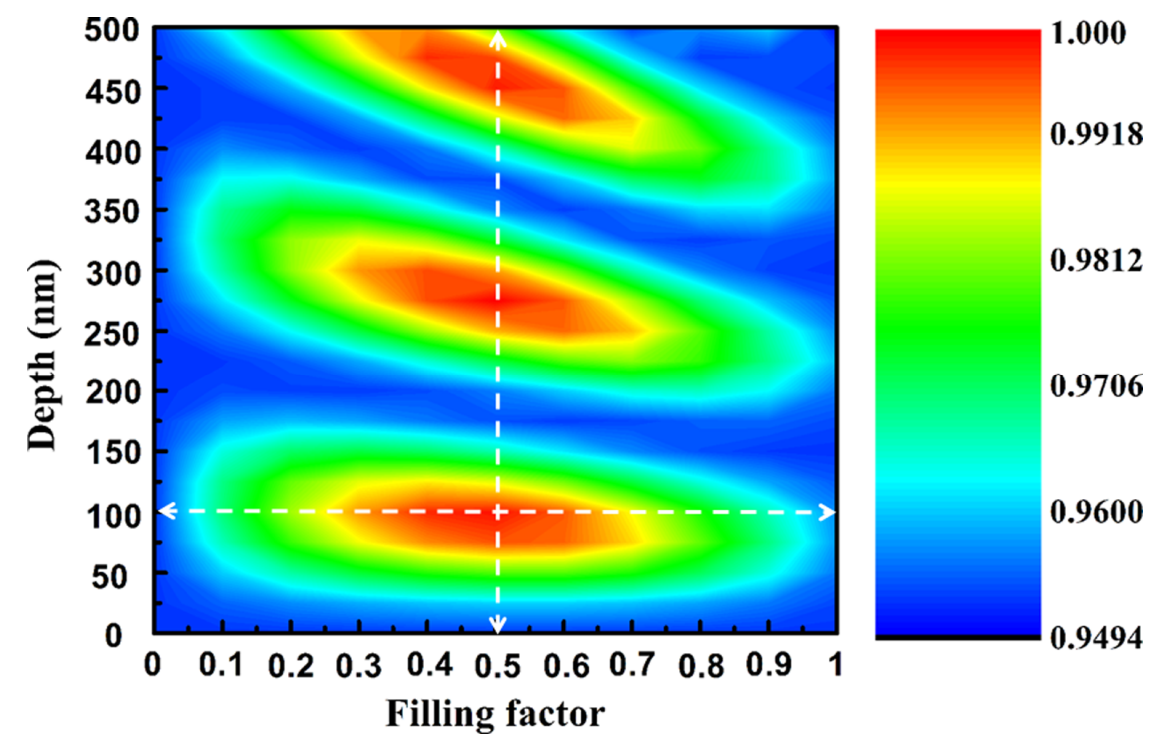

Figure 4.2 Peking University RCWA simulation results. Transmission at normal direction as a function of grating depth and filling factor. 


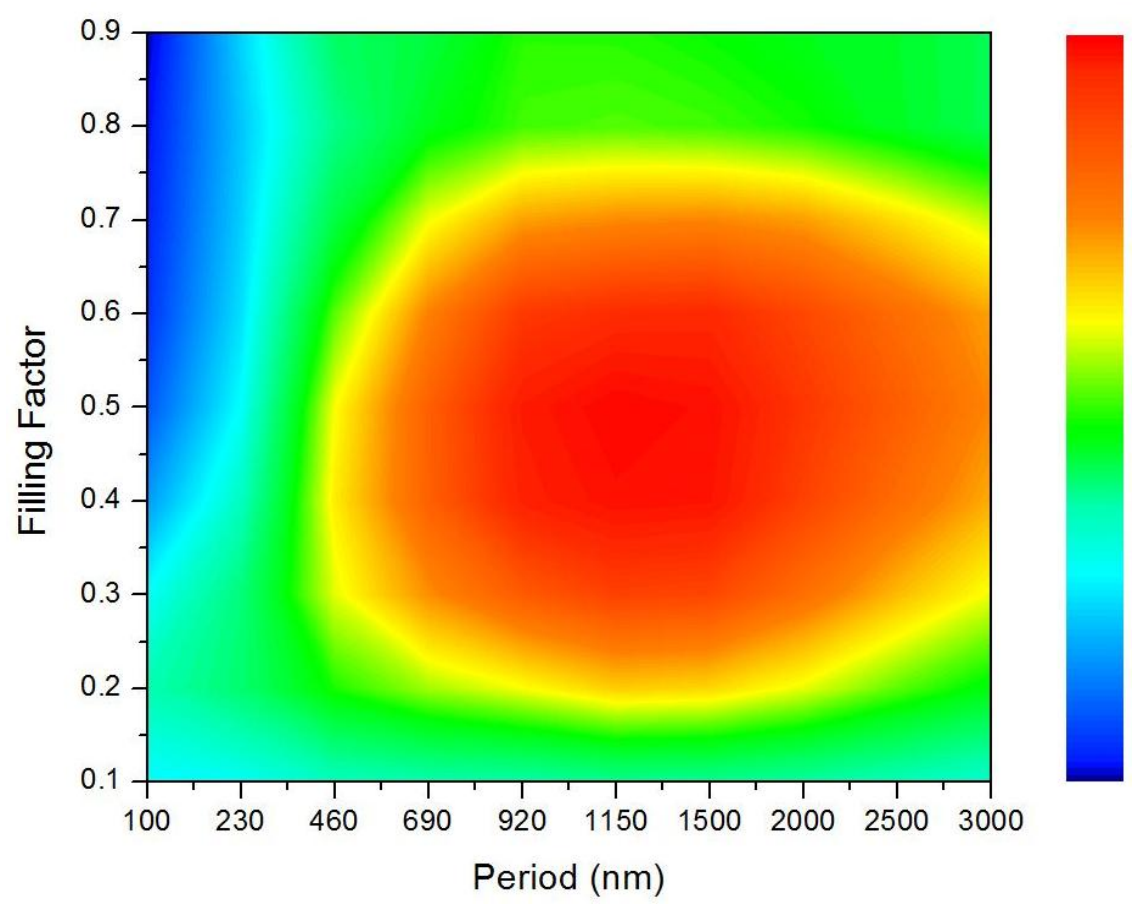

Figure 4.3 Peking University RCWA simulation results. Transmission as a function of filling factor and grating period.

\subsection{FDTD Analysis}

\subsubsection{ITO and GaN top grating}

First, we compare the difference between ITO and GaN top gratings emitting into either free space or epoxy resin to validate that increasing the critical angle also increases the amount of light extracted. Table 4-2 lists the critical angle for different combinations of grating material and surround medium. As explained earlier, a larger critical angle improves extraction efficiency by allowing more light to escape the LED. Hence, the combination of ITO and Epoxy should extract the most light. Each simulation model is based on the description in Table 4-1 and Figure 4.1. In order to reduce simulation time and complexity, we use a sapphire substrate thickness of $10 \mu \mathrm{m}$ instead of $80 \mu \mathrm{m}$ and we do not include the Ag reflection layer. The simulation results (Figure 4.4) show that larger critical angles increase light extraction. Compared to the GaN to Air case, ITO to 
Air enhances light extraction by $11 \%, \mathrm{GaN}$ to Epoxy by $36 \%$, and ITO to Epoxy by $40 \%$ showing that critical angle does play a significant role in light extraction efficiency.

Table 4-2 Critical angle based on surrounding medium and grating material.

\begin{tabular}{|l|l|l|l|}
\hline Grating & $\begin{array}{l}\text { Surrounding } \\
\text { Medium }\end{array}$ & $\begin{array}{l}\text { Critical } \\
\text { Angle }\end{array}$ & $\begin{array}{l}\text { \% Improvement } \\
\text { compared to GaN to Air }\end{array}$ \\
\hline ITO & Air & 28.4 & 11 \\
\hline ITO & Epoxy & 48.0 & 40 \\
\hline GaN & Air & 23.5 & 0 \\
\hline GaN & Epoxy & 38.6 & 36 \\
\hline
\end{tabular}

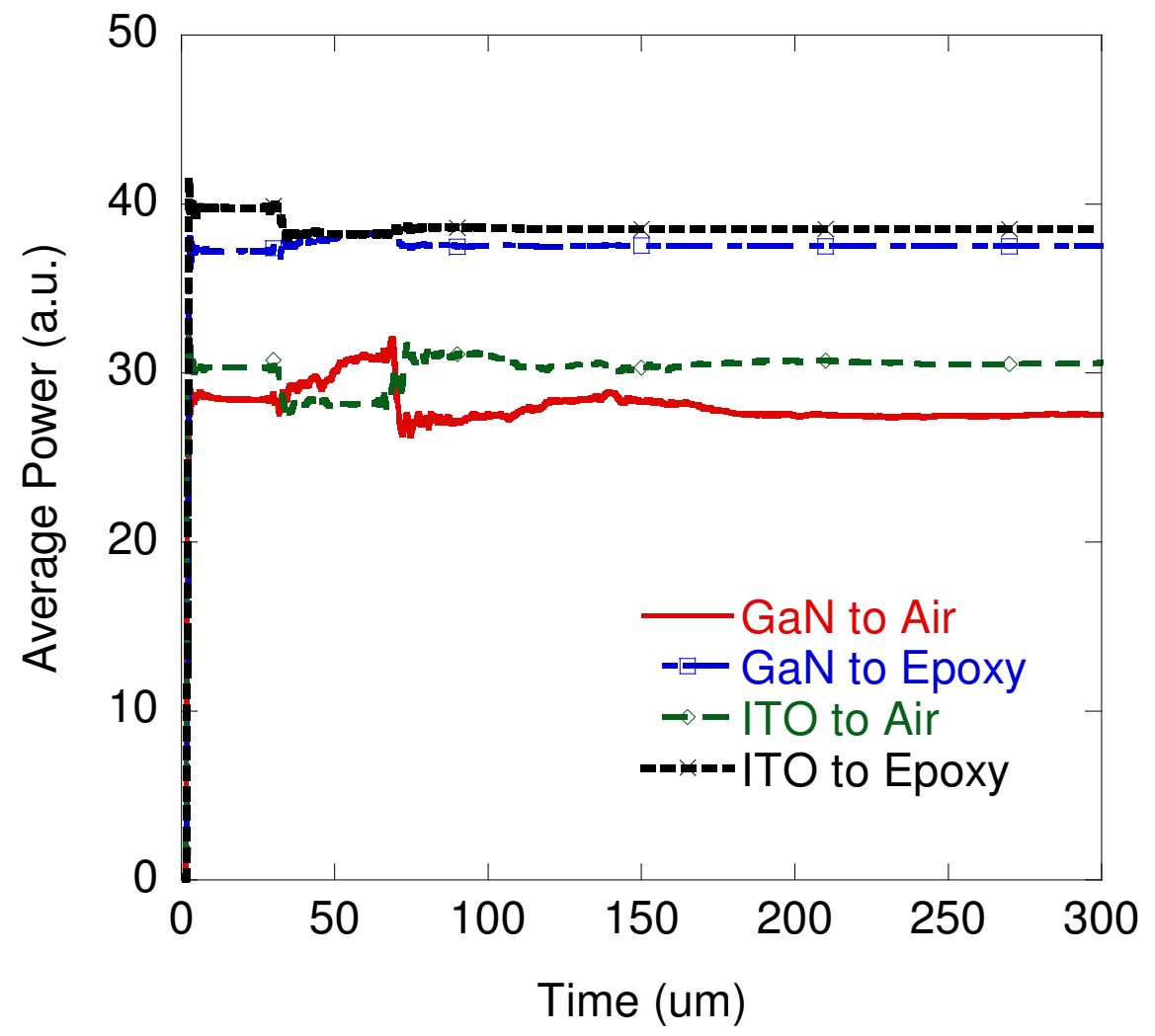

Figure 4.4 Light extraction of GaN to air, GaN to Epoxy, ITO to air, and ITO to Epoxy. 


\subsubsection{Optimizing grating period}

Next, we run simulations to find the optimal ITO grating period with and without the Ag reflection layer. With an epoxy background material increase the period incrementally from 100-1500nm. Figure 4.5 shows average power of light extracted versus grating period. Similar to Peking University's RCWA results, low light transmission occurs in the $100-690 \mathrm{~nm}$ range and high transmission occurs for larger grating periods in the 690 - 1500nm range. Unlike the RCWA analysis, these results also show that using an Ag reflection layer increases overall light transmission. Other research also supports that using a reflection layer increases light extraction by up to $73 \% .{ }^{26}$ According to .

Table 4-3 the reflection layer increases light extraction for every case except when the grating period equals $100 \mathrm{~nm}$. The table also shows that light extraction continues to increase as grating period increases. 


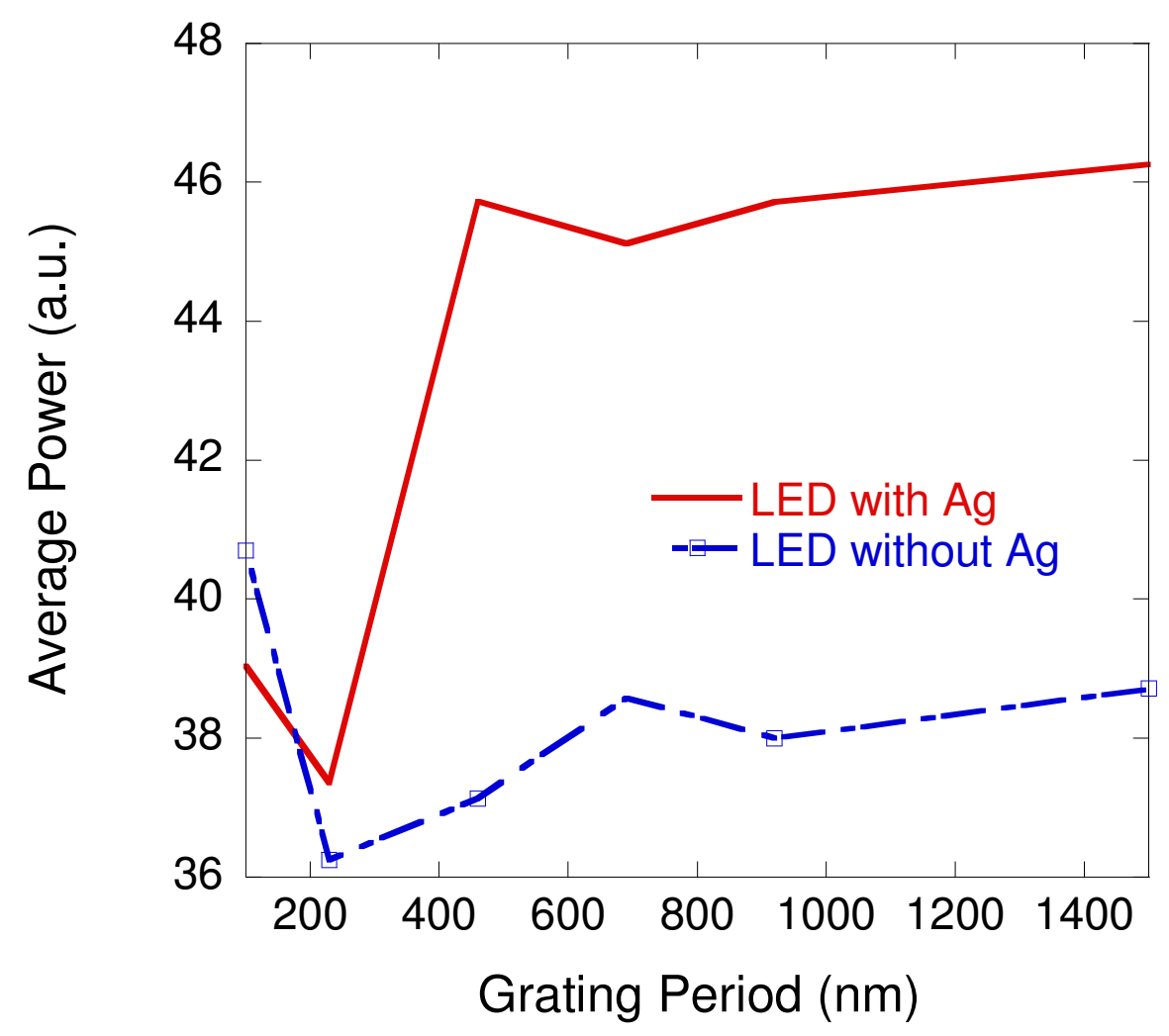

Figure 4.5 Average power vs. grating period for an LED with and without a silver reflection layer. The grating is ITO and the background is epoxy.

Table 4-3 Increased light extraction for LEDs with and without an Ag reflection layer.

\begin{tabular}{|l|l|l|l|}
\hline $\begin{array}{l}\text { Grating Period } \\
(\mathrm{nm})\end{array}$ & $\begin{array}{l}\text { Average Power } \\
\text { without Ag (a.u.) }\end{array}$ & $\begin{array}{l}\text { Average Power } \\
\text { with Ag (a.u.) }\end{array}$ & $\begin{array}{l}\% \\
\text { Increase }\end{array}$ \\
\hline 100 & 40.703 & 39.043 & -4.08 \\
\hline 230 & 36.243 & 37.358 & 3.08 \\
\hline 460 & 37.13 & 45.721 & 23.14 \\
\hline 690 & 38.577 & 45.118 & 16.96 \\
\hline 920 & 37.997 & 45.711 & 20.30 \\
\hline 1130 & 38.514 & 45.78 & 18.87 \\
\hline 1500 & 38.712 & 46.269 & 19.52 \\
\hline
\end{tabular}

\subsubsection{ITO top grating with Ag reflection layer}

Finally, we analyze in more detail the effects of the Ag reflection layer by simulating four structures: (1) a conventional LED with no silver and no grating, (2) a conventional LED with silver at the bottom of the sapphire substrate, (3) an LED with an ITO grating 
period $=1130 \mathrm{~nm}$, and $(4)$ an LED with ITO grating period $=1130 \mathrm{~nm}$ and silver on the bottom layer. Each structure is simulated with a sapphire thickness of $10 \mu \mathrm{m}$ and $80 \mu \mathrm{m}$ to observe the light enhancing effects of sapphire thickness. Figure 4.6 and Table 4-4 show that for a sapphire substrate thickness of $10 \mu \mathrm{m}$, the LEDs with the Ag reflection layer extract $18 \%$ more light compared to an LED without the reflection layer. Conversely, Figure 4.7 shows that with an $80 \mu \mathrm{m}$ sapphire substrate thickness, the silver reflection layer decreases light extraction by $26 \%$.

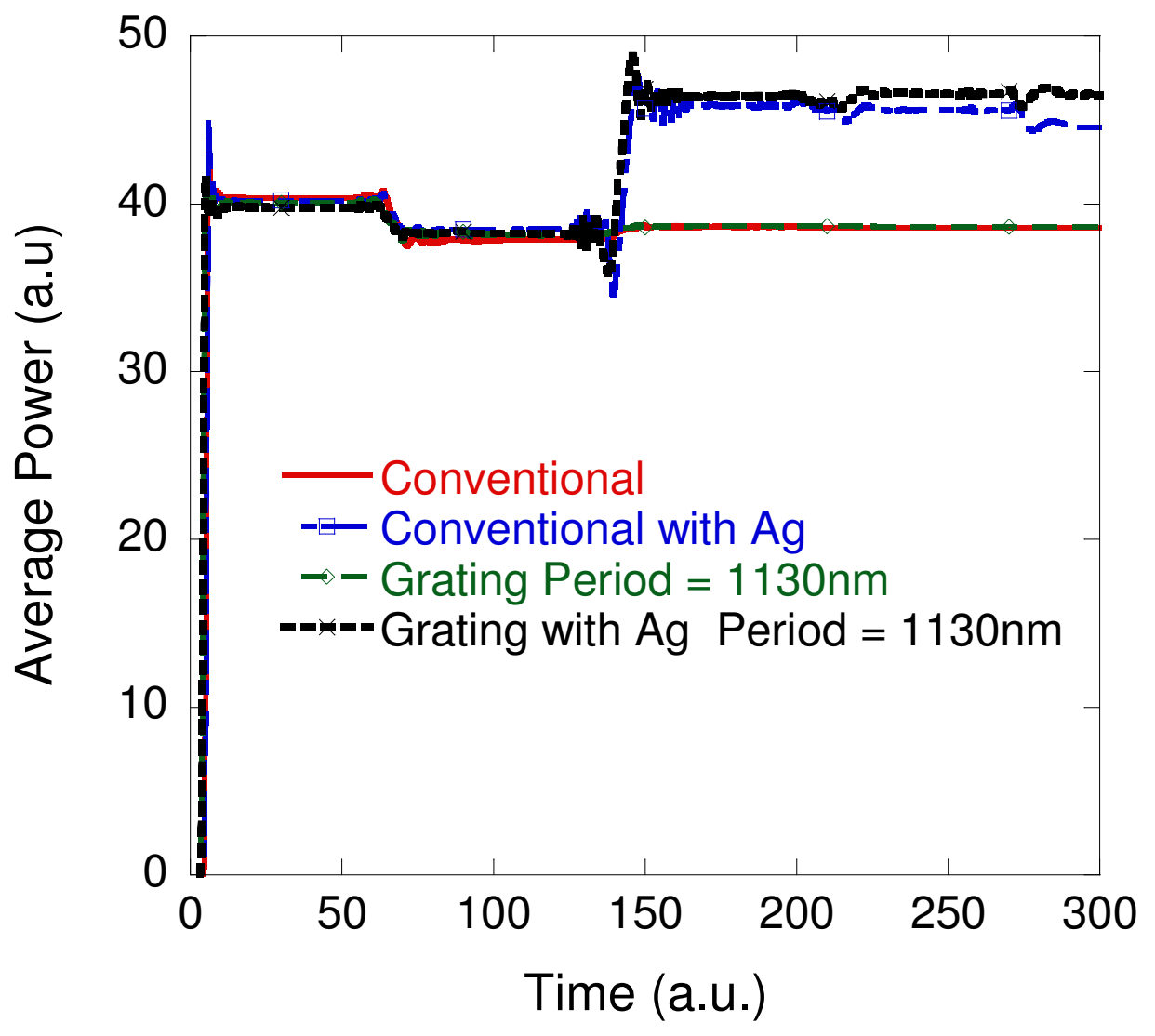

Figure 4.6 Average power extracted plotted against time when sapphire substrate equals $10 \mu \mathrm{m}$. 
Table 4-4 Average power extracted for four different structures when sapphire substrate is $10 \mu \mathrm{m}$.

\begin{tabular}{|c|c|c|c|}
\hline LED Structure & $\begin{array}{c}\text { Average Power } \\
\text { without } \operatorname{Ag(a.u.)}\end{array}$ & $\begin{array}{c}\text { Average Power with } \\
\operatorname{Ag}(\text { a.u. })\end{array}$ & $\begin{array}{c}\text { Percent Increase } \\
(\%)\end{array}$ \\
\hline Conventional & 38.60 & 43.25 & 12.04 \\
\hline ITO Grating & 38.63 & 45.78 & 18.51 \\
\hline
\end{tabular}

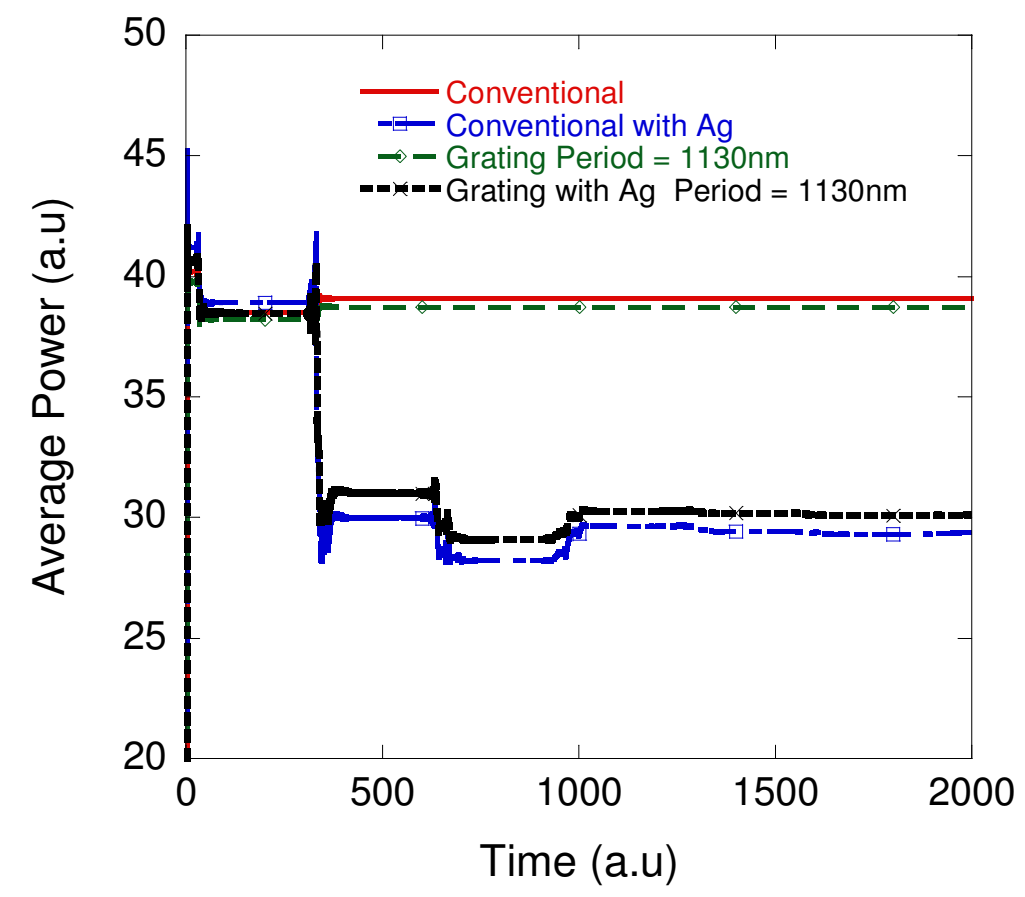

Figure 4.7 Average power extracted plotted against time when sapphire substrate thickness is $80 \mu \mathrm{m}$.

Table 4-5 Average power extracted for four different structures when sapphire substrate is $80 \mu \mathrm{m}$.

\begin{tabular}{|c|c|c|c|}
\hline $\begin{array}{c}\text { LED } \\
\text { Structure }\end{array}$ & $\begin{array}{c}\text { Average Power } \\
\text { without } \operatorname{Ag}(\text { a.u. })\end{array}$ & $\begin{array}{c}\text { Average Power } \\
\text { with Ag (a.u.) }\end{array}$ & $\begin{array}{c}\text { Percent Increase } \\
(\%)\end{array}$ \\
\hline Conventional & 39.07 & 29.37 & -24.83 \\
\hline ITO Grating & 38.74 & 30.11 & -22.28 \\
\hline
\end{tabular}

The simulations with different sapphire substrate thickness gave drastically different results. To further investigate these differences, we simulated all four structures and varied the sapphire substrate thickness from $10 \mu \mathrm{m}$ to $80 \mu \mathrm{m}$ in steps of $10 \mu \mathrm{m}$. The plot of average power versus sapphire substrate thickness in Figure 4.8 shows that the average power extracted increases and decreases periodically as a function of substrate thickness 
only for LEDs with the reflection grating. The increase and decrease in average power could be a result of standing waves forming in the LED or light exiting the LED from the sides instead of the top. No papers have been found that claim similar findings.

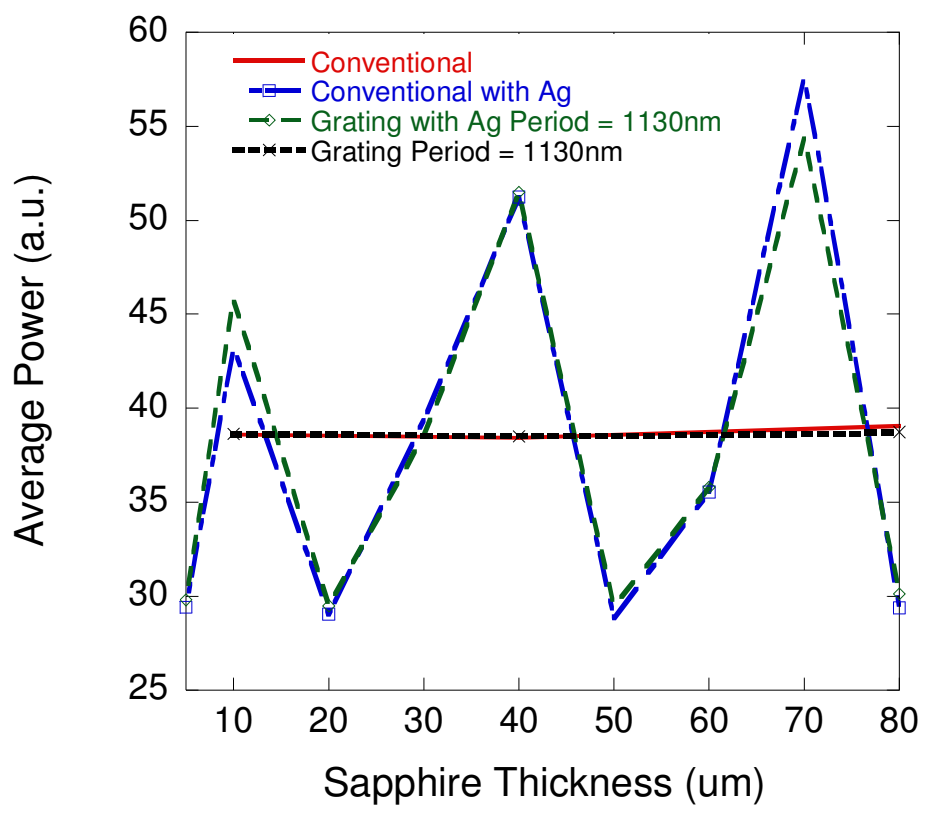

Figure 4.8 Average power vs. sapphire thickness for four different structures: (1) conventional, (2) conventional with $\mathrm{Ag}$ reflection layer, (3) ITO grating, and (4) ITO grating with Ag reflection layer.

\subsection{Conclusion}

This chapter successfully shows that increasing the critical angle and implementing an Ag reflection layer improves overall light extraction efficiency. First, increasing the critical angle from 23.5 to 47.9 degrees using an ITO top grating and an Epoxy dome improved light extraction by $40 \%$. Next, in agreement with Peking University's RCWA simulations, increasing the ITO grating period increases light extraction. Finally, we show that light extraction for structures containing Ag reflection layers is highly dependent on sapphire substrate thickness. We investigate this trend further in Chapter 6 of this report. 


\section{CHAPTER 5: PATTERNED SAPPHIRE SUBSTRATE AND SIO ${ }_{2}$ ARRAY}

Chapter 3 and Chapter 4 discuss using nanoscale top gratings to enhance external quantum efficiencies. This chapter examines strategies that affect both internal and external quantum efficiencies. GaN LEDs grown on sapphire substrates suffer from threading dislocation defects due to the crystal lattice and thermal expansion mismatch between GaN and sapphire usually on the order of $10^{9}-10^{11} \mathrm{~cm}^{-2} \cdot{ }^{27}$ Comparatively, GaAs exhibits $10^{2}-10^{4}$ dislocations $\mathrm{cm}^{-2}$ and homoepitaxial Silicon has practically no dislocations. Threading dislocations greatly reduce optical performance and LED lifetime. The method of epitaxial lateral overgrowth drastically reduces threading dislocations to the order $\sim 10^{7} \mathrm{~cm}^{-2}$. This approach masks off the highly dislocated GaN region, stops GaN growth, and restarts growth on selective layers forcing it to grow laterally in a purer crystal form. Epitaxial lateral overgrowth can be implemented using patterned sapphire substrates (PSS) and $\mathrm{SiO}_{2}$ nanorod arrays. Both PSS and $\mathrm{SiO}_{2}$ arrays are fabricated using nanoimprint lithography in a four step process as follows: ${ }^{28}$

(1) Deposit the $\mathrm{SiO}_{2}$ or Sapphire and spin coat a 200nm polymer layer.

(2) Place a patterned mold onto the polymer film and applying high pressure until heating the polymer above the glass transition temperature.

(3) Allow the sample to cool to room temperature.

(4) Use reactive ion etching (RIE) with $\mathrm{BCL}_{3}, \mathrm{CF}_{4}$, and $\mathrm{O}_{2}$. $\mathrm{BCL}_{3}$ transfers the pattern onto the sapphire, $\mathrm{CF}_{4}$ transfers the pattern onto $\mathrm{GaN}$ and $\mathrm{O}_{2}$ removes the polymer mask.

These two structures improve both internal and external quantum efficiencies. Internal quantum efficiencies are improved by suppressing threading dislocations. $\mathrm{SiO}_{2}$ 
arrays decrease dislocation density in four ways shown in Figure 5.1. First, voids between nanorods bend dislocations and eventually develop into stacking faults. Second, stacking faults block growth of dislocations. Third, the formation of voids in the case of slow GaN growth rates stops dislocation growth. Finally, residual $\mathrm{SiO}_{2}$ inhibits $\mathrm{GaN}$ growth and reduces dislocation formation. ${ }^{29}$ Similar to top gratings, the periodicity and shape of patterned sapphire substrates and $\mathrm{SiO}_{2}$ arrays enhances light extraction by scattering light. This chapter focuses on optimizing the light scattering properties of an LED structure with a PSS and $\mathrm{SiO}_{2}$ nanorod array. Again, the FDTD method is used to simulate light extraction enhancement.
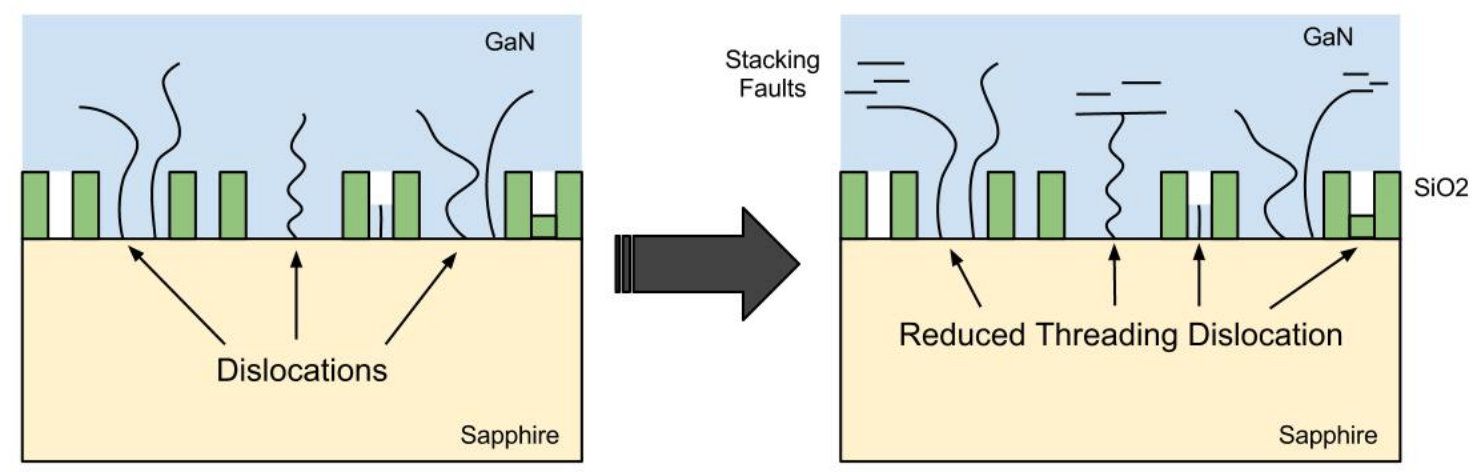

Figure 5.1 Example of stacking faults blocking threading dislocations adapted from National Chiao-Tung University et al. $^{29}$

\subsection{Simulation Model}

Previous studies show that in comparison with a conventional LED structure, a PSS alone improves light output power by $35 \%$ and a PSS in combination with a $\mathrm{SiO}_{2}$ photonic quasi-crystal (PQC) improves light output power by $48 \% .^{28}$ The four structures we simulate include: (1) a conventional LED, (2) an LED with only a PSS, (3) an LED with only a $\mathrm{SiO}_{2}$ nanorod array, and (4) an LED with both a PSS and $\mathrm{SiO}_{2}$ nanorod array. 
Although a $\mathrm{SiO}_{2} \mathrm{PQC}$ and nanorod array have the same function and purpose, they do not take on the same shape. A PQC exhibits long range order and short range disorder ${ }^{30}$ which cannot be simulated in two dimensions. For this reason we model the array as a simple $2 \mathrm{PhC}$ grating in two dimensions. Table 5-1 below provides a description of the conventional LED including material type, thickness, and refractive index. Table 5-2 shows the simulation parameters used in the FDTD analysis. In this section of the chapter we first attempt to match the results of the study that achieved $35 \%$ and $48 \%$ improvements in output power. Next, we optimize patterned sapphire substrate width and period. Finally we optimize the $\mathrm{SiO}_{2}$ array by changing its position in the z-direction. Figure 5.2 and Figure 5.3 are both diagrams of the LED simulated. Figure 5.2 provides labels for each layer of the LED described in Table 5-1. Figure 5.3 helps define important parameters of the LED such as PSS period (d), width (w), and the $\mathrm{z}$ position of the $\mathrm{SiO}_{2}$ array.

Table 5-1 Conventional GaN LED Parameters

\begin{tabular}{|c|c|c|}
\hline Material & $\begin{array}{c}\text { Thickness } \\
(\mu \mathrm{m})\end{array}$ & $\begin{array}{c}\text { Refractive } \\
\text { Index }\end{array}$ \\
\hline $\mathrm{p}-\mathrm{GaN}$ & 0.12 & 2.55 \\
\hline $\mathrm{P}-\mathrm{AlGaN}$ & 0.05 & 2.5 \\
\hline $\mathrm{InGaN} / \mathrm{GaN}$ & 0.115 & 2.6 \\
\hline $\mathrm{n}-\mathrm{GaN}$ & 2 & 2.55 \\
\hline $\mathrm{GaN}$ & 3 & 2.55 \\
\hline Sapphire & 4 & 1.77 \\
\hline
\end{tabular}

Table 5-2 Simulation setup parameters

\begin{tabular}{|c|c|c|c|}
\hline$\Delta \mathrm{x}(\mu \mathrm{m})$ & $\Delta \mathrm{z}(\mu \mathrm{m})$ & $\begin{array}{c}\text { Time Step } \\
(\mu \mathrm{m})\end{array}$ & PML $(\mu \mathrm{m})$ \\
\hline 0.03 & 0.03 & 0.02 & 0.5 \\
\hline
\end{tabular}




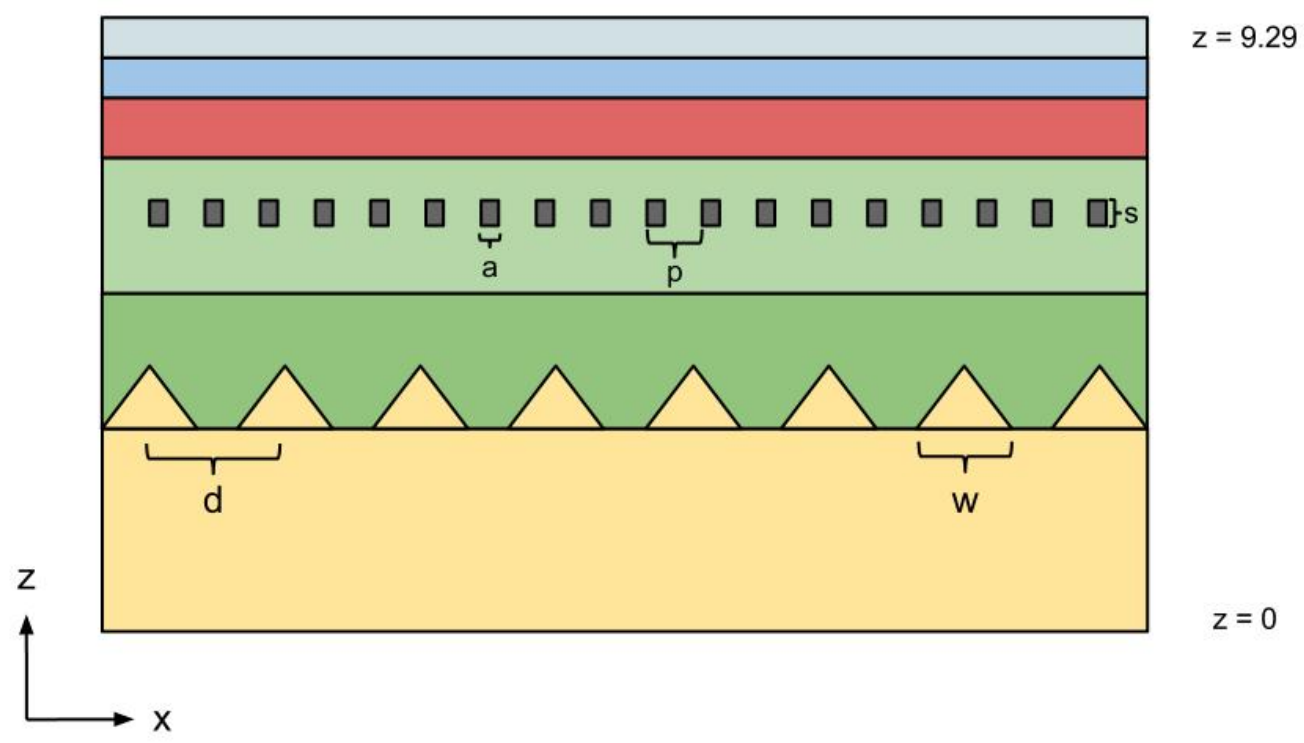

Figure 5.2 Labeled diagram of LED with PSS and $\mathrm{SiO}_{2}$ array.

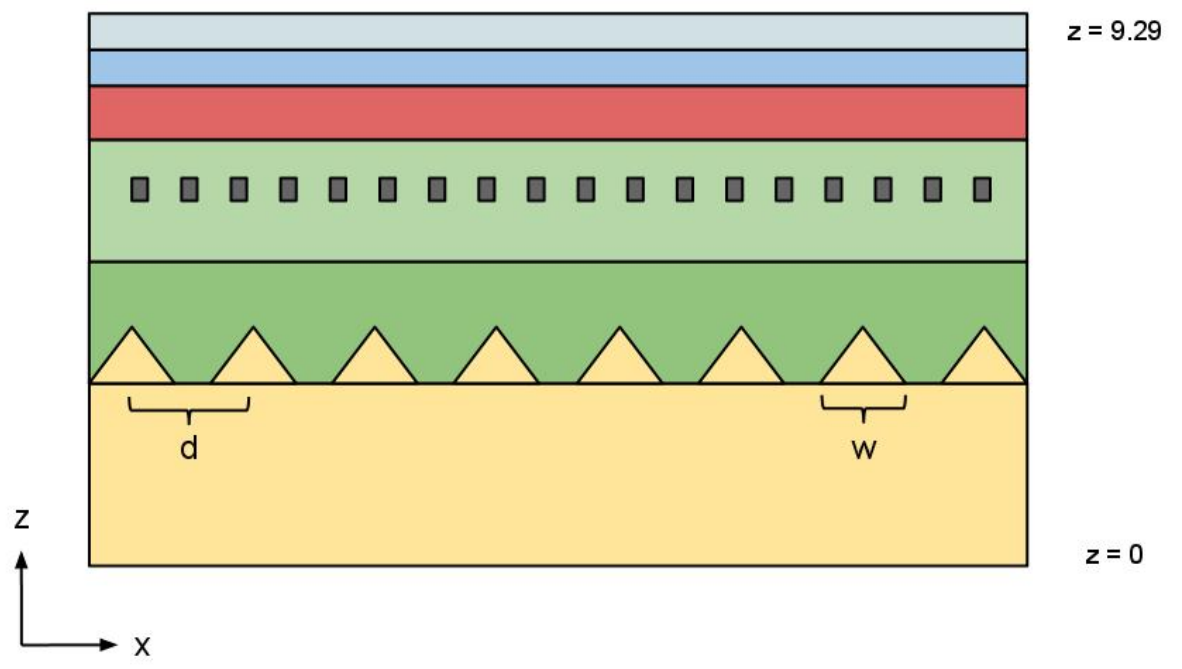

Figure 5.3 Diagram of LED with PSS and $\mathrm{SiO}_{2}$ array. The diagram defines the PSS period (d), width (w), and shows the $x$ and $\mathrm{z}$ direction of the LED.

\subsection{Matching Previous Studies}

Previous light extraction measurements show that a PSS alone improves light output power by $35 \%$ and the combination of $\mathrm{PSS}$ and $\mathrm{SiO}_{2}$ array improves light output power by $48 \% .{ }^{28}$ To confirm these results we use the FDTD method to simulate light extraction 
plotted against time for a conventional LED, an LED with a PSS, an LED with a $\mathrm{SiO}_{2}$ nanorod array, and an LED with both PSS and $\mathrm{SiO}_{2}$ array. The PSS period $\mathrm{d}=3 \mu \mathrm{m}$ and width $\mathrm{w}=2.5 \mu \mathrm{m}$. The $\mathrm{SiO}_{2}$ nanorod width $\mathrm{a}=400 \mathrm{~nm}$, period $\mathrm{p}=750 \mathrm{~nm}$, depth $\mathrm{s}=$ 200nm and is centered at $\mathrm{z}=7.8 \mu \mathrm{m}$. Figure 5.3 illustrates these dimensions. The results displayed in Figure 5.4, show a 15\% improvement for the PSS structure and a $26 \%$ improvement for the PSS \& $\mathrm{SiO}_{2}$ array structure with the $\mathrm{SiO}_{2}$ centered at $\mathrm{z}=7.8 \mu \mathrm{m}$. The results do not perfectly match those of the previous study but they do show similar trends. For example, both PSS and $\mathrm{SiO}_{2}$ nanorod structures enhance light extraction and the structure with both PSS and $\mathrm{SiO}_{2}$ nanorod array increases light extraction more than the simulation only containing the PSS. A summary of the results including percent improvement compared to the conventional LED are listed below in .

Table 5-3.

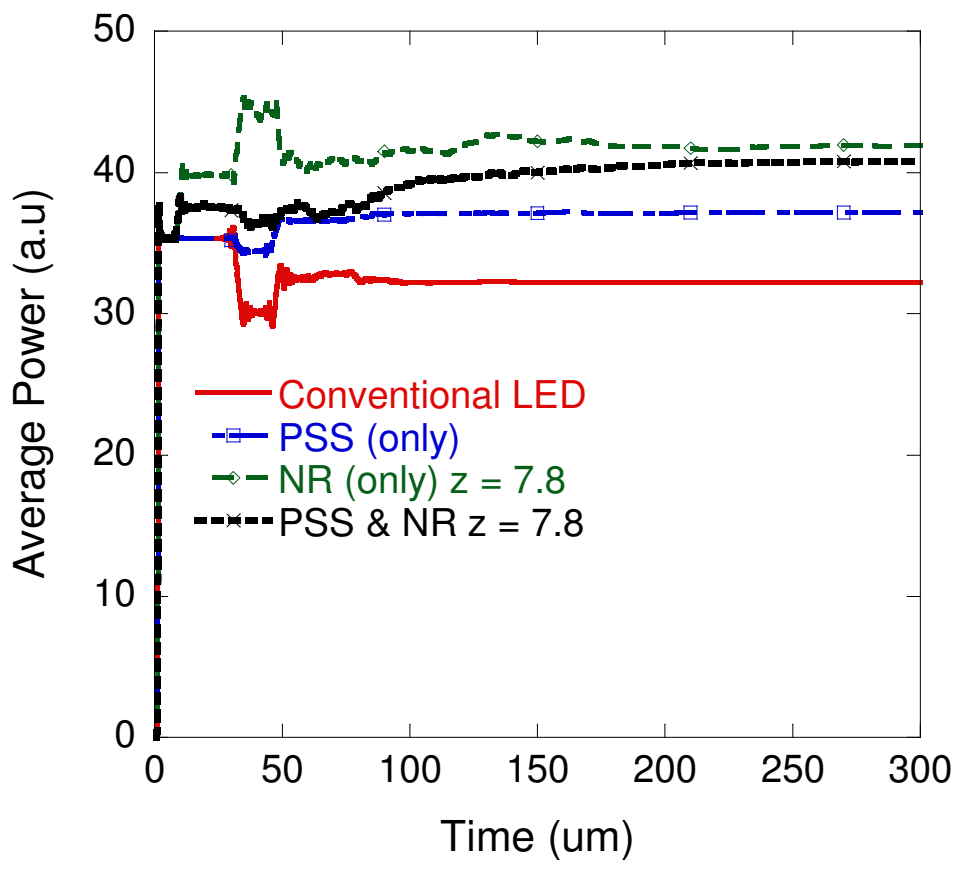


Figure 5.4 Average power of light extracted in four structures: conventional LED, patterned sapphire substrate, $\mathrm{SiO} 2$ nanorod, and patterned sapphire substrate with $\mathrm{SiO}_{2}$ nanorod PSS width w $=2.5 \mu \mathrm{m}$ and period $\mathrm{d}=3 \mu \mathrm{m} . \mathrm{SiO}_{2}$ layer is centered at $\mathrm{z}=7.8$ $\mu \mathrm{m}$.

Table 5-3 Light extraction improvement compared to the conventional LED.

\begin{tabular}{|c|c|c|}
\hline Structure & $\begin{array}{c}\text { Average } \\
\text { Power } \\
\text { (a.u.) }\end{array}$ & $\begin{array}{c}\text { Percent } \\
\text { Improvement }\end{array}$ \\
\hline Conventional & 32.239 & $0 \%$ \\
\hline PSS (only) & 37.159 & $15 \%$ \\
\hline NR (only) & 41.938 & $30 \%$ \\
\hline PSS \& NR & 40.769 & $26 \%$ \\
\hline
\end{tabular}

\subsection{Patterned Sapphire Substrate Optimization}

Next, we optimize the patterned sapphire substrate by changing the period (d) from $2 \mu \mathrm{m}$ to $3 \mu \mathrm{m}$ in steps of $0.5 \mu \mathrm{m}$ and width (w) from 1 to $2.5 \mu \mathrm{m}$ in steps of $0.1 \mu \mathrm{m}$. Figure 5.5 shows that the maximum average power occurs when $\mathrm{w}=1.3 \mu \mathrm{m}$ and $\mathrm{d}=2 \mu \mathrm{m}$. A disadvantage of choosing this point is that a small change in PSS width results in a large change in average power. This leaves little room for error in the device fabrication process. A more stable point would be where $\mathrm{w}=2.5 \mu \mathrm{m}$ and $\mathrm{d}=3 \mu \mathrm{m}$. .

Table 5-4 summarizes the simulations performed and includes the light extraction percent improvement compared to the conventional LED. The structure only containing a PSS (w $=1.3 \mu \mathrm{m}$ and $\mathrm{d}=2 \mu \mathrm{m}$ ) improves light extraction by $47.9 \%$. This is the largest improvement observed compared to the conventional LED thus far. 


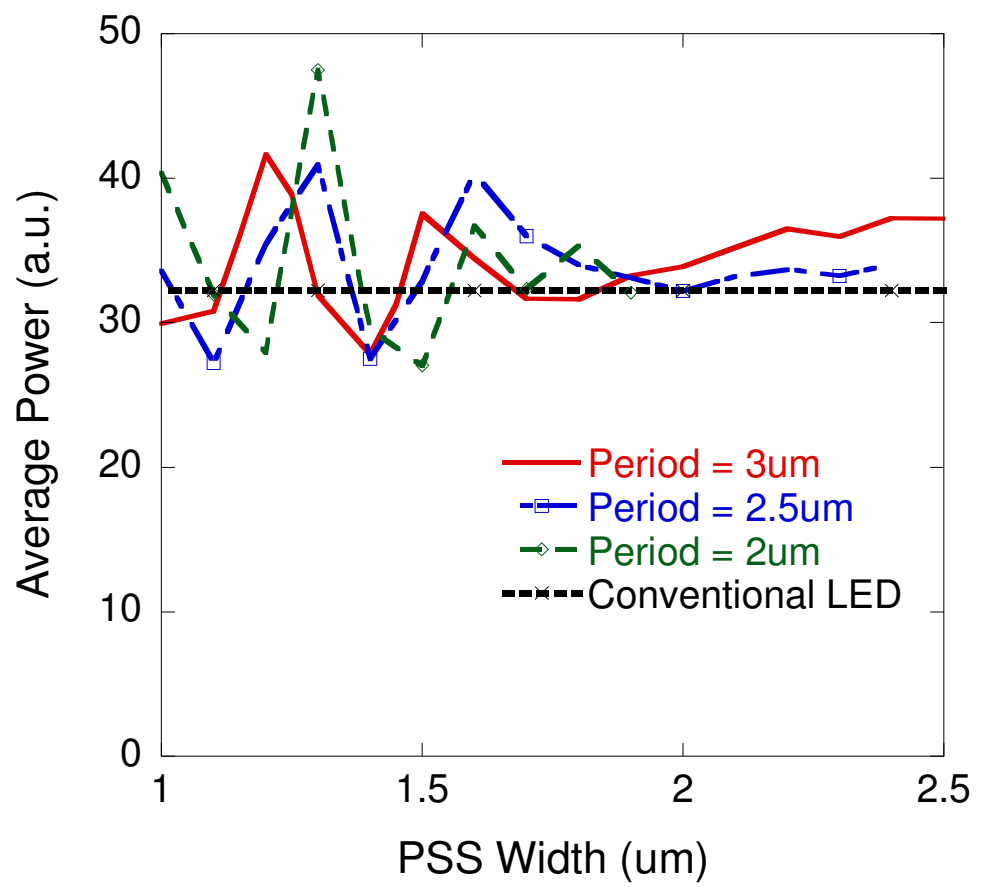

Figure 5.5 Average power as a function of patterned sapphire substrate (PSS) width and PSS period.

Table 5-4 Light extraction improvement by changing PSS width (w) and period (d).

\begin{tabular}{|c|c|c|}
\hline Structure & $\begin{array}{c}\text { Max Average } \\
\text { Power (a.u.) }\end{array}$ & $\begin{array}{c}\text { Percent } \\
\text { Improvement }\end{array}$ \\
\hline Conventional & 32.24 & $0 \%$ \\
\hline $\begin{array}{c}\mathrm{d}=2 \mu \mathrm{m} \\
\mathrm{w}=1.3 \mu \mathrm{m}\end{array}$ & 47.68 & $47.9 \%$ \\
\hline $\begin{array}{c}\mathrm{d}=2.5 \mu \mathrm{m} \\
\mathrm{w}=1.3 \mu \mathrm{m}\end{array}$ & 40.93 & $26.9 \%$ \\
\hline $\begin{array}{c}\mathrm{d}=3 \mu \mathrm{m} \\
\mathrm{w}=1.2 \mu \mathrm{m}\end{array}$ & 41.65 & $29.2 \%$ \\
\hline
\end{tabular}

\subsection{Optimizing $\mathrm{SiO}_{2}$ Array}

Next, we optimize the position of $\mathrm{SiO}_{2}$ array by moving it up and down in the $\mathrm{z}$ direction between $7 \mu \mathrm{m}$ and $8 \mu \mathrm{m}$. Implementing this method, we optimized three separate structures: (1) an LED with a $\mathrm{SiO}_{2}$ array and no PSS, (2) an LED with both $\mathrm{SiO}_{2}$ and PSS $(\mathrm{d}=3 \mathrm{um}$ and $\mathrm{w}=2.5 \mathrm{um})$, and (3) an LED with both $\mathrm{SiO}_{2}$ and PSS $(\mathrm{d}=2 \mathrm{um}$ and 
$\mathrm{w}=1.3 \mathrm{um}$ ). Figure 5.6 shows the average power as a function of $\mathrm{SiO}_{2}$ position for each of these structures.

Table 5-5 highlights the effect of changing PQC position, and shows that light extraction improves by $51.8 \%$ for the second structure when $\mathrm{z}=7.1 \mathrm{um}, \mathrm{d}=3 \mathrm{um}$, and $\mathrm{w}=2.5 \mathrm{um}$.

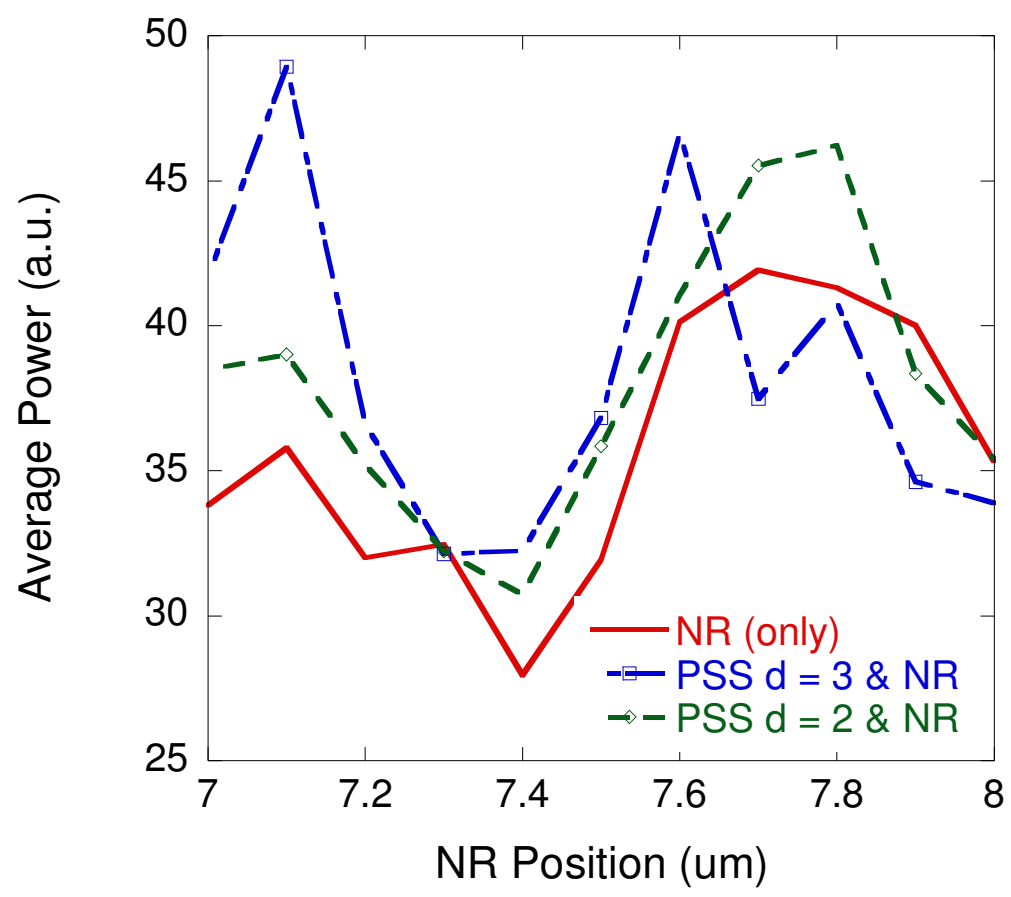

Figure 5.6 Average power as a function of nanorod array position. In the case with a PSS, $\mathrm{d}=3 \mathrm{um}, \mathrm{w}=2.5 \mathrm{um}$, and $\mathrm{d}=$ $2 \mathrm{um}$ and $\mathrm{w}=1.3 \mathrm{um}$.

Table 5-5 Light extraction improvement by changing $\mathrm{SiO}_{2}$ array position.

\begin{tabular}{|c|c|c|}
\hline Structure & $\begin{array}{c}\text { Max Average } \\
\text { Power (a.u.) }\end{array}$ & $\begin{array}{c}\text { Percent } \\
\text { Improvement }\end{array}$ \\
\hline Conventional & 32.24 & $0 \%$ \\
\hline $\begin{array}{c}\text { NR only } \\
\mathrm{z}=7.7 \mu \mathrm{m}\end{array}$ & 41.93 & $30 \%$ \\
\hline $\begin{array}{c}\text { PSS d }=3 \mu \mathrm{m} \& \\
\text { NR z }=7.1 \mu \mathrm{m}\end{array}$ & 48.93 & $51.8 \%$ \\
\hline $\begin{array}{c}\text { PSS d }=2 \mu \mathrm{m} \& \\
\text { NR z }=7.1 \mu \mathrm{m}\end{array}$ & 46.23 & $43.4 \%$ \\
\hline
\end{tabular}




\subsection{Conclusion}

In this chapter we adjust patterned sapphire substrate width and period and $\mathrm{SiO}_{2}$ z-position to optimize light extraction. First, we confirm the results from a previous study. The simulation results in Figure 5.4 do not perfectly match the results of Huang et al. for two possible reasons. First, they measured the light extraction of a physical device while we simulated the light extraction of that device. Their measurement took into account all three dimensions of the device. The above simulation was performed in 2-D. Second, their $\mathrm{SiO}_{2}$ nanorod array takes the shape of a photonic quasi crystal (PQC). A 2-D simulation cannot represent the complexities of a PQC. Instead we use a simple $2 \mathrm{PhC}$ structure for the $\mathrm{SiO}_{2}$ array. Second, setting the period and width to $2 \mu \mathrm{m}$ and $1.3 \mu \mathrm{m}$ respectively increases light extraction by $47.9 \%$. Finally, optimizing the z-position of the $\mathrm{SiO}_{2}$ nanorod array to $7.1 \mu \mathrm{m}$ increases light extraction by $51.8 \%$. In the next chapter we investigate the same structures with the use of an $\mathrm{Ag}$ reflection layer. 


\section{CHAPTER 6: PSS, SIO 2 ARRAY AND AG REFLECTION LAYER}

After optimizing the LED via changing PSS width and period and $\mathrm{SiO}_{2}$ nanorod position, we improve upon the structure by including an Ag reflection layer. Typically GaN based LEDs use light absorbing bonding pads such as $\mathrm{Ti} / \mathrm{Au}$ and $\mathrm{Cr} / \mathrm{Au}$ metals. These pads cover up to $10 \%$ of wafer surface area, absorb significant amounts of light and reduce output efficiency. Alternatively Ag based electrodes improve light extraction by reflecting a maximum of $95 \%$ light at the blue wavelength. ${ }^{31}$ Ag reflection layers are becoming increasingly popular for GaN-based vertical LEDs. Experiments show Agbased mirrors increasing light output by up to $17 \% .{ }^{32}$ Pure Ag is not a fitting p-ohmic contact due to its low work function and poor adhesion properties. Recent studies show that $\mathrm{Ag}$ sandwiched between Ni cladding layers improves adhesion to $\mathrm{p}-\mathrm{GaN}$ and remains highly reflective (93\%). ${ }^{32}$ Other studies also show the feasibility of using Ag as an ohmic-contact with $\mathrm{n}-\mathrm{GaN}^{33}$ and sapphire substrates. ${ }^{26}$ A group in Taiwan shows that using an $\mathrm{Ag} / \mathrm{Cr} / \mathrm{Au}$ n-type electrode in combination with a PSS improves light output by $40 \%$ compared to a conventional LED. ${ }^{34}$ In this chapter, we use Ag as a reflection layer between the sapphire substrate and $\mathrm{n}-\mathrm{GaN}$ layer as well as a reflection layer below the sapphire substrate. First we discuss how to properly use Ag in the FullWAVE software. Second, we describe the simulation model and examine problems encountered in the process of simulating. Third, we analyze the effect of using Ag as a reflector above and below the sapphire substrate. Finally, we optimize the height of the sapphire substrate and consider the effects on light extraction. 


\subsection{Using AG in FullWAVE}

Unlike GaN, Sapphire, and $\mathrm{SiO}_{2}$, the refractive index and permittivity of Silver changes as a function of frequency. Rsoft's FullWAVE software provides a material editor to define the linear or non-linear permittivity and permeability of different materials. The editor utilizes either a linear or nonlinear sum of multiple resonance terms shown in equation 6.1. The Debye, Lorentzian, and Drude models can all be used to define permittivity and permeability. An example of the Lorentzian equation is shown below in equation 6.2 . To properly define the material, the user must provide $a_{k}, b_{k}$, and $c_{k}$ values.

$$
\begin{aligned}
& \varepsilon(\omega)=\varepsilon_{\infty}+\sum_{k} \varepsilon_{k}(\omega) \\
& \varepsilon_{k}(\omega)=\frac{\Delta \varepsilon_{k}}{-a_{k}-b_{k}(i \omega)+c_{k}}
\end{aligned}
$$

To utilize this feature, right click on the desired material. In the properties box, click on the "Materials..." button to open the Material Editor. Click "New Material" to create and define the coefficients $a_{k}, b_{k}$, and $c_{k}$. Finally, when using a linear or nonlinearly dispersive material check the Dispersion/Nonlinearity box in the FullWAVE Simulation Parameters window. Checking this box enables the use of such materials. Figure 6.1 provides a screen shot of the material editor along with the dispersion coefficient values that define Silver. Figure 6.2 shows both real and imaginary refractive index of silver as a function of wavelength. Notice the extreme nonlinearities of both the imaginary and real indices. 


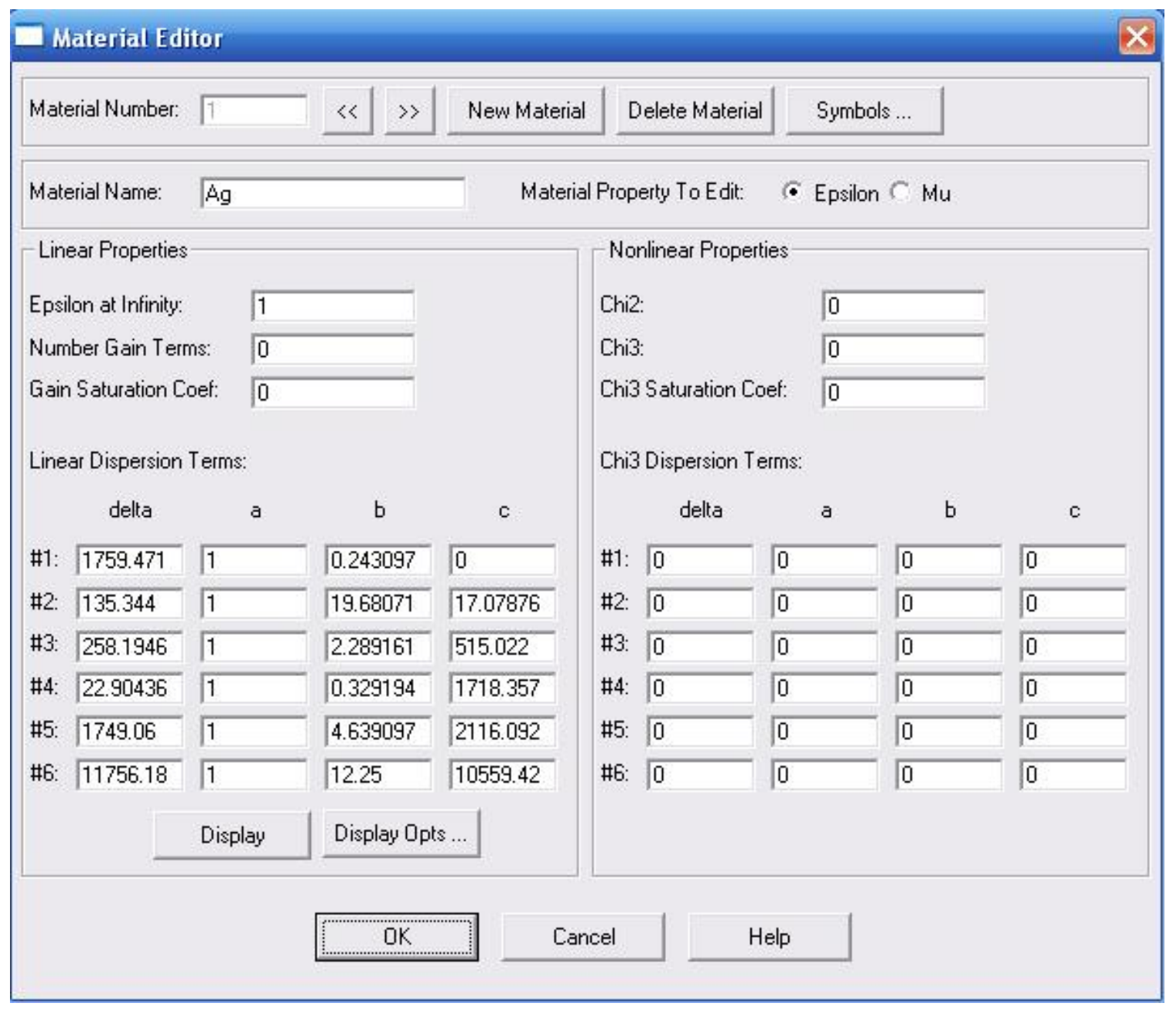

Figure 6.1 Material editor displaying the properties used for Ag. 


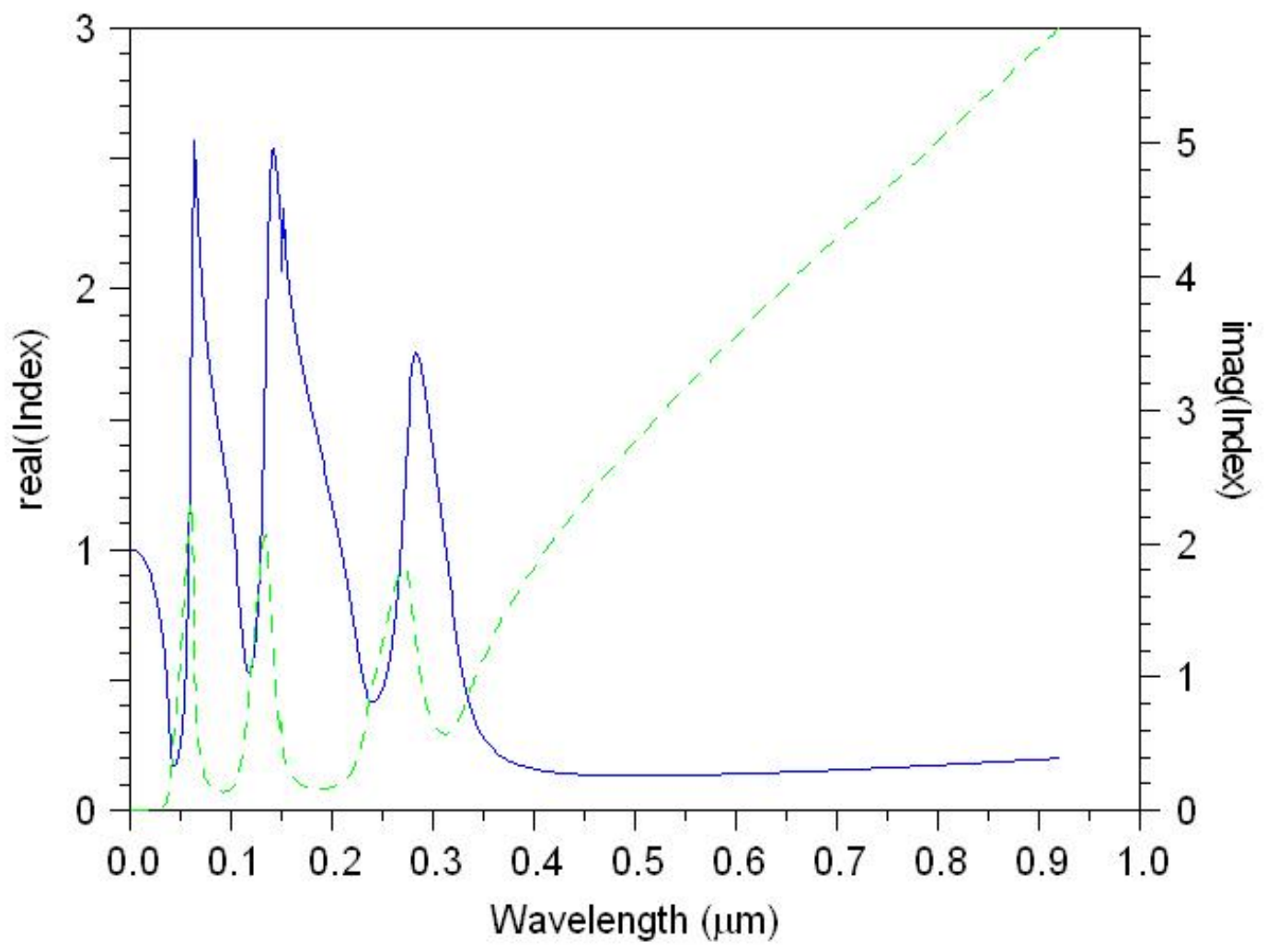

Figure 6.2 Real (blue) and imaginary (green) refractive index for Ag.

\subsection{Simulation Model}

Next, we improve upon Chapter 5's LED model by adding an Ag reflection layer either above the sapphire substrate or below the sapphire substrate. Figure 6.3 shows the LED structure with a reflection grating between the sapphire and n-GaN layer. Figure 6.4 shows the same structure with the reflection layer on the bottom of the sapphire substrate. Figure 6.4 also includes a new parameter " $\mathrm{H}$ " to describe the thickness of the sapphire substrate. All other parameters of the LED remain identical to the structure described in

Table 5-3 with the exception of the $1 \mathrm{~nm}$ thick Ag reflection layer. In every set of simulations we simulate and compare all four structures: (1) Conventional LED, (2) LED 
with PSS only, (3) LED with PSS and $\mathrm{SiO}_{2}$ nanorods, and (4) LED with both PSS and $\mathrm{SiO}_{2}$ nanorods.

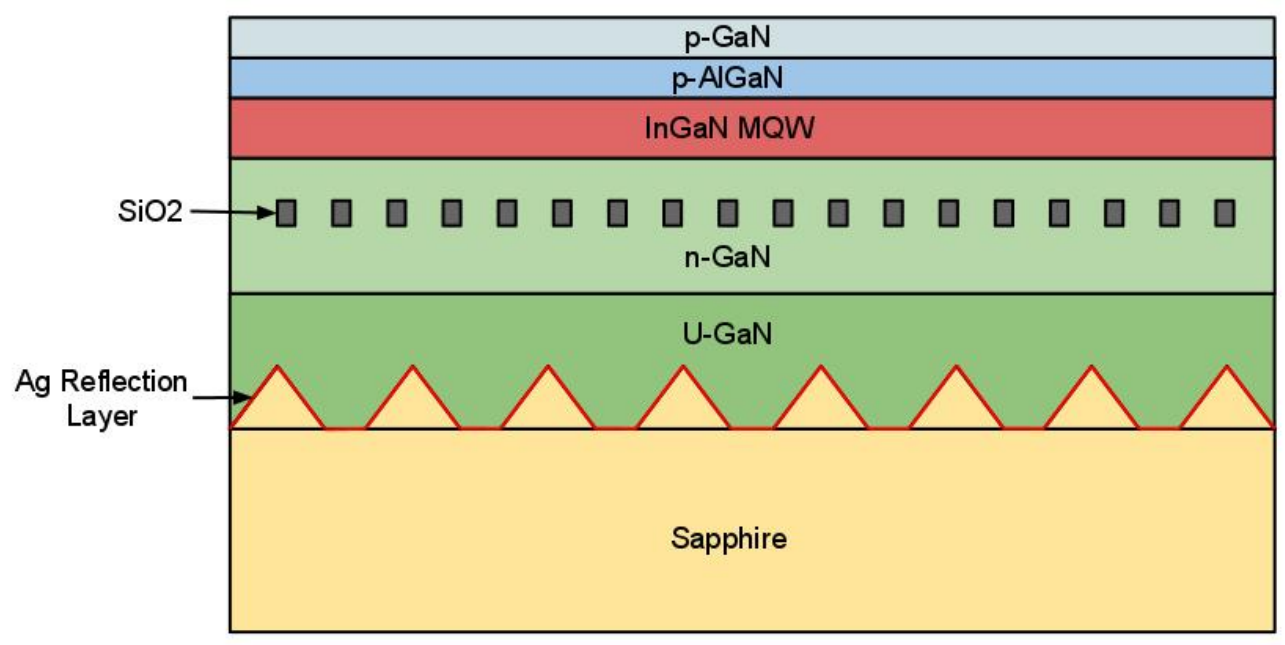

Figure 6.3 LED with Ag reflection layer between sapphire substrate and un-doped GaN.

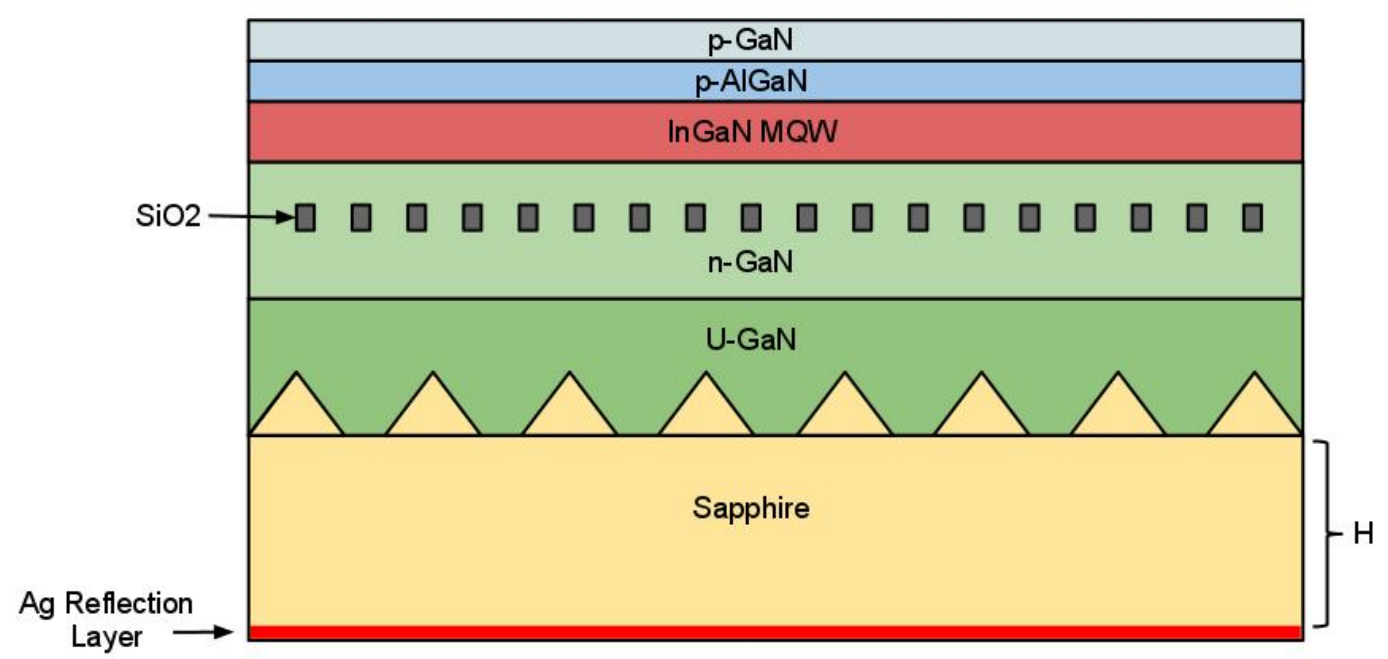

Figure 6.4 LED with Ag reflection layer below the sapphire substrate. The variable $\mathrm{H}$ represents sapphire substrate height. 


\subsubsection{Problems encountered with the software}

Unexpectedly, the first simulation ran continuously; i.e. the simulation did not complete. Closer inspection of the optical field distribution in Figure 6.5 and Figure 6.6 reveals an error in the simulation. Figure 6.5 shows the distribution of a previous simulation. The colors range from red to blue. Red represents the highest intensity and blue represents the lowest intensity. Figure 6.6 shows the optical distribution of a simulation containing Ag. The distribution contains traces of red, blue, and green but has been taken over by black and white as a result of extremely large gain in the material.

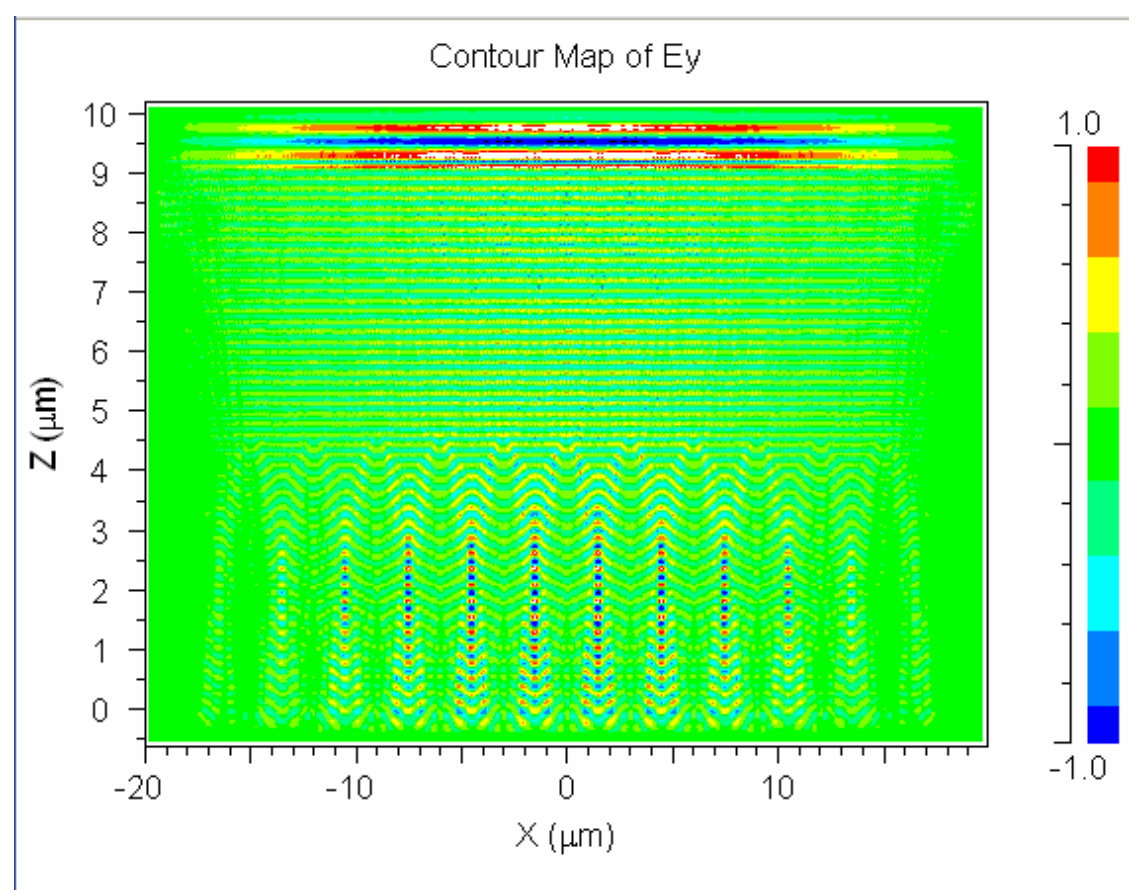

Figure 6.5 Optical field distribution of an LED without Ag. 


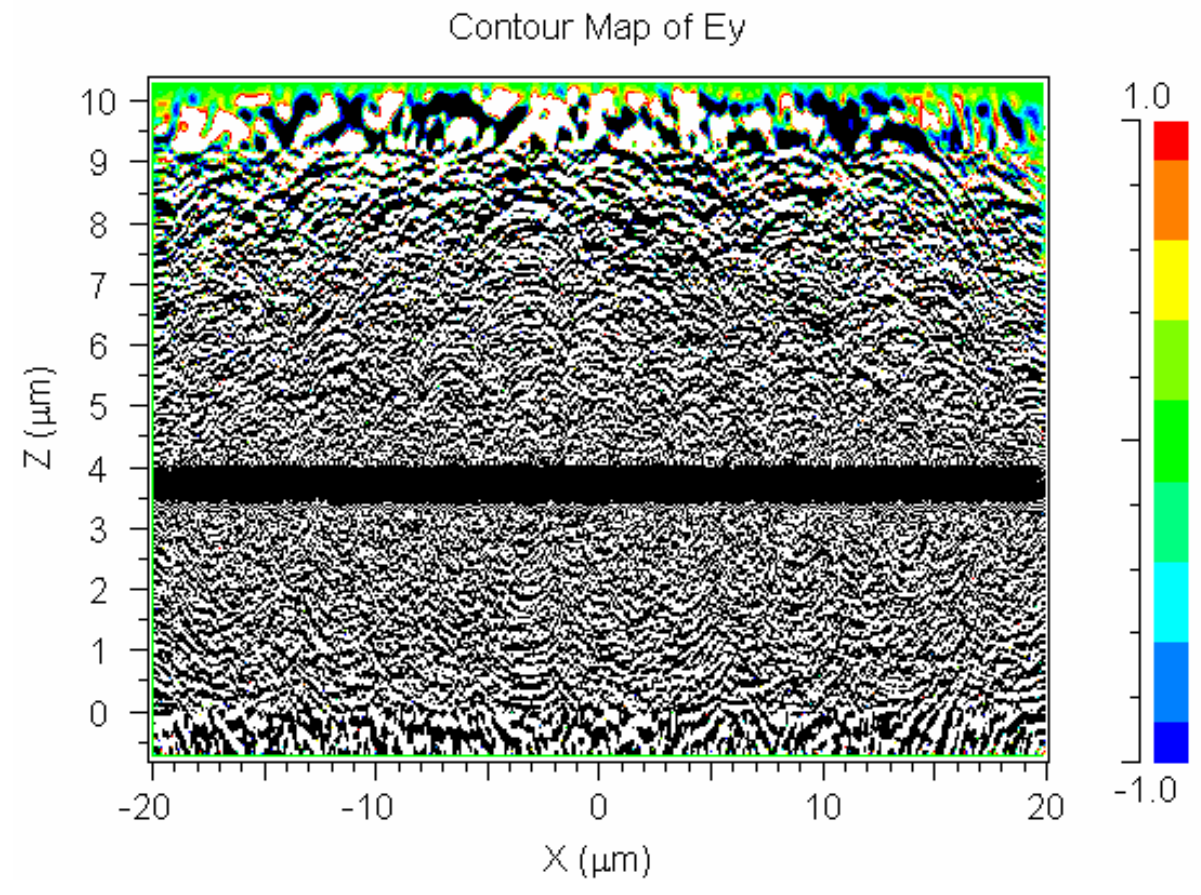

Figure 6.6 Optical field distribution of an LED with an $\mathrm{Ag}$ reflection layer.

We hypothesize the unexpected and undesired gain results from too coarse of a grid size.

To confirm this hypothesis, Figure 6.7 shows grid overlap at the interface between Ag and GaN. The red line represents the interface between two materials. It is possible that the field gets trapped when it oversteps the boundary into the Ag layer and builds up energy as time passes. 


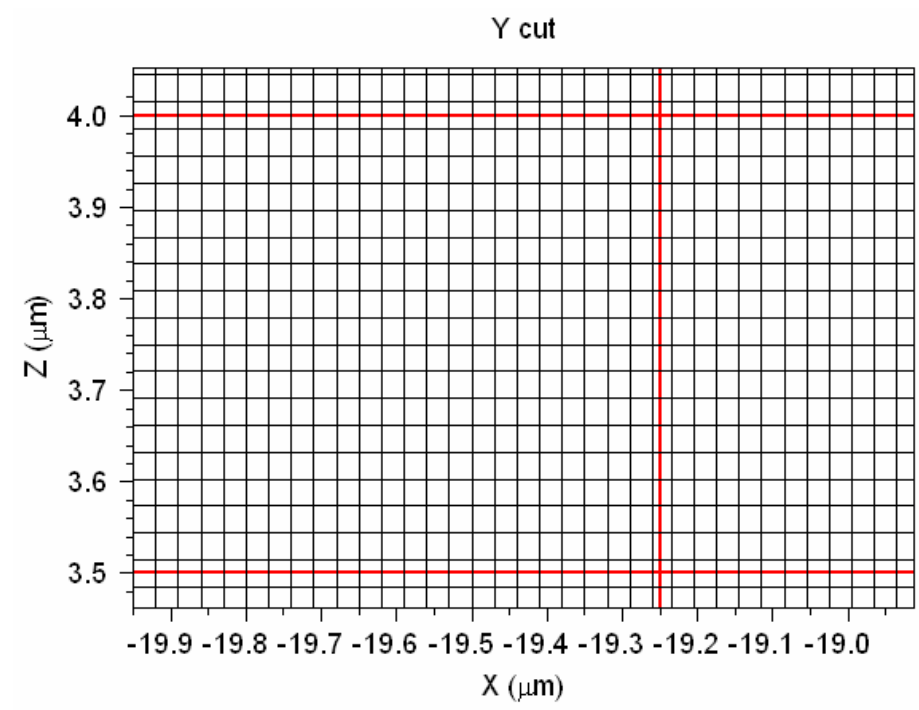

Figure 6.7 Uniform grid size with overlap between $\mathrm{Ag}$ and GaN interface.

We attempt two methods to resolve this issue. First, align the grid with the material boundary to eliminate any overlap. Second, reduce the size of the spatial and temporal dimensions. To align the grid with the material boundary click the "perform simulation" stoplight shaped button. In the box titled "advanced grid control" check the "enable nonuniform" box. Clicking "grid options" allows the user to edit the interface alignment. Changing the alignment to "none," "centered," and "straddled" did not improve the gain issues. Figure 6.8 shows the realigned grid spacing with no overlap between the material boundaries. 


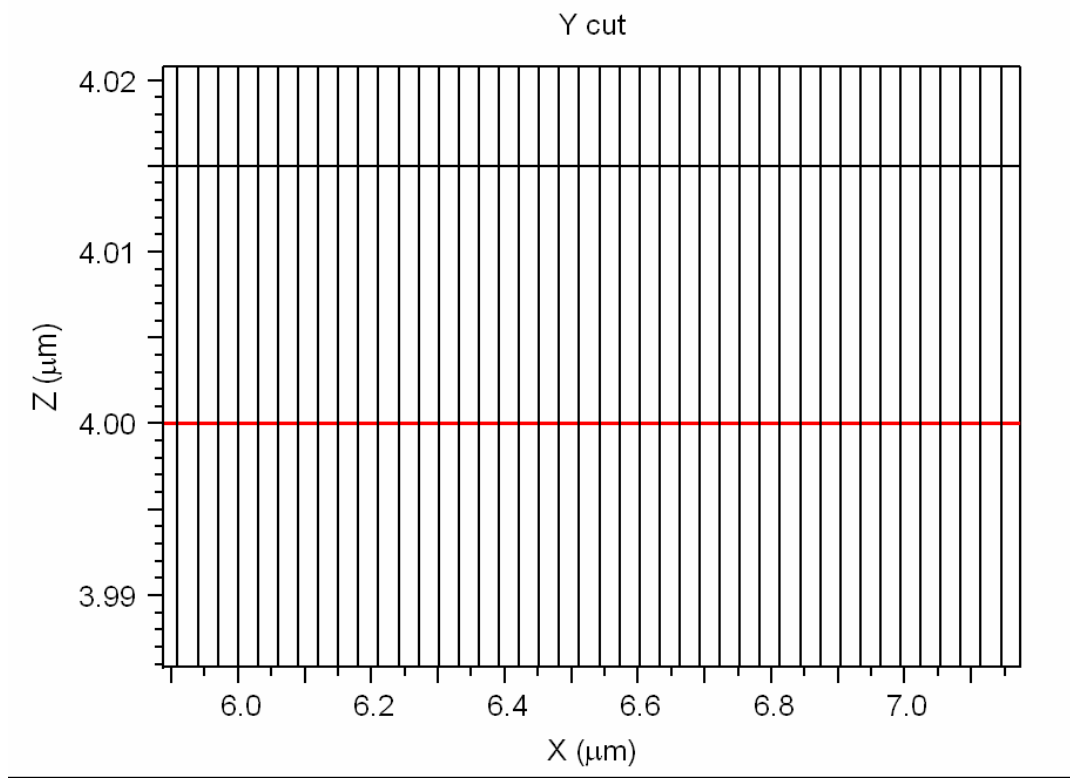

Figure 6.8 Realigned grid spacing.

Reducing the spatial grid size in both the $\mathrm{x}$ and $\mathrm{z}$ dimensions also did not improve the gain issues, however reducing the temporal grid size eliminated all gain errors. The following simulations performed in this chapter use the simulation parameters in Table 6-1. These simulation parameters remain exactly the same as those in Table 5-1 and Table 5-2 except the time step decreases from 0.02 to 0.01 .

Table 6-1 Simulation parameters used with Ag reflection layer.

\begin{tabular}{|c|c|c|c|}
\hline$\Delta \mathrm{x}(\mu \mathrm{m})$ & $\Delta \mathrm{z}(\mu \mathrm{m})$ & $\begin{array}{c}\text { Time Step } \\
(\mu \mathrm{m})\end{array}$ & PML $(\mu \mathrm{m})$ \\
\hline 0.03 & 0.03 & 0.01 & 0.5 \\
\hline
\end{tabular}

\subsubsection{Silver between PSS and n-GaN}

After resolving Ag gain problems, we continue our simulations. The first set of simulations includes a conventional LED, an LED with a PSS, an LED with a $\mathrm{SiO}_{2}$ nanorod array, and finally an LED with both a PSS and a $\mathrm{SiO}_{2}$ array. Each of these structures contains a reflection grating between the Sapphire and GaN interface. If the sapphire is patterned, the Ag layer takes on the same pattern as shown in Figure 6.3. 
Figure 6.9 shows the average power of light extracted with the Ag reflection layer sandwiched between the sapphire substrate and un-doped GaN layers for all four structures.

Table 6-2 compares the average power of these four structures with and without the Ag reflection layer. The column labeled percent improvement compares the LED structure with and without the Ag reflector. For example, the conventional LED with the Ag reflector extracts less light than the conventional LED without the reflector and reduces extraction by $28.47 \%$.

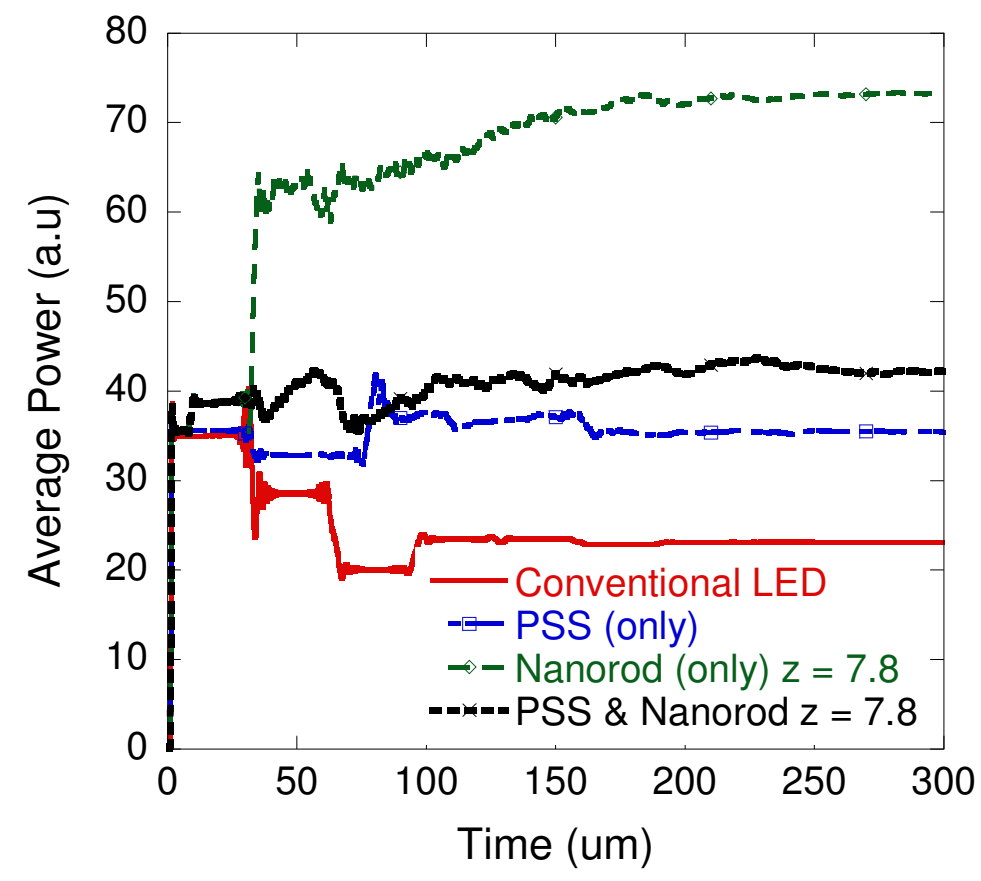

Figure 6.9 Average power of light extracted in four structures with an $\mathrm{Ag}$ reflection layer between $\mathrm{u}-\mathrm{GaN}$ and Sapphire Substrate: conventional LED, patterned sapphire substrate, $\mathrm{SiO} 2$ 
$\mathrm{PhC}$, and patterned sapphire substrate with $\mathrm{SiO} 2$ nanorod. PSS width $\mathrm{w}=2.5 \mu \mathrm{m}$ and period $\mathrm{d}=3 \mu \mathrm{m}$. $\mathrm{SiO}_{2}$ layer is centered at $\mathrm{z}=7.8 \mu \mathrm{m}$.

Table 6-2 Percent improvement of Ag layer compared to LEDs without an $\mathrm{Ag}$ layer between $\mathrm{u}-\mathrm{GaN}$ and sapphire substrate.

\begin{tabular}{|c|c|c|c|}
\hline Structure & $\begin{array}{c}\text { Average } \\
\text { Power (a.u.) }\end{array}$ & $\begin{array}{c}\text { Average Power } \\
\text { (a.u.) with Ag } \\
\text { Reflection Layer }\end{array}$ & $\begin{array}{c}\text { Percent } \\
\text { Improvement } \\
(\%)\end{array}$ \\
\hline Conventional & 32.239 & 23.061 & -28.47 \\
\hline PSS (only) & 37.159 & 35.489 & -4.49 \\
\hline NR (only) & 41.938 & 73.375 & 75 \\
\hline PSS \& NR & 40.769 & 42.215 & 3.55 \\
\hline
\end{tabular}

\subsection{Optimizing Sapphire Substrate Height}

Finally we simulated four structures with a bottom layer Ag reflector. To optimize these structures, we sweep sapphire height from $10 \mu \mathrm{m}$ to $80 \mu \mathrm{m}$ in steps of $10 \mu \mathrm{m}$. Figure 6.10 shows the results of changing the sapphire height. Maximum total light extraction occurs for the conventional structure with sapphire height equal to $40 \mu \mathrm{m}$. Compared to the conventional LED without $\mathrm{Ag}$, there is a $160 \%$ increase in total light extraction. Surprisingly, the LED structure with both PSS and $\mathrm{SiO}_{2}$ nanorods only improves by $34 \%$ compared to a conventional LED. 


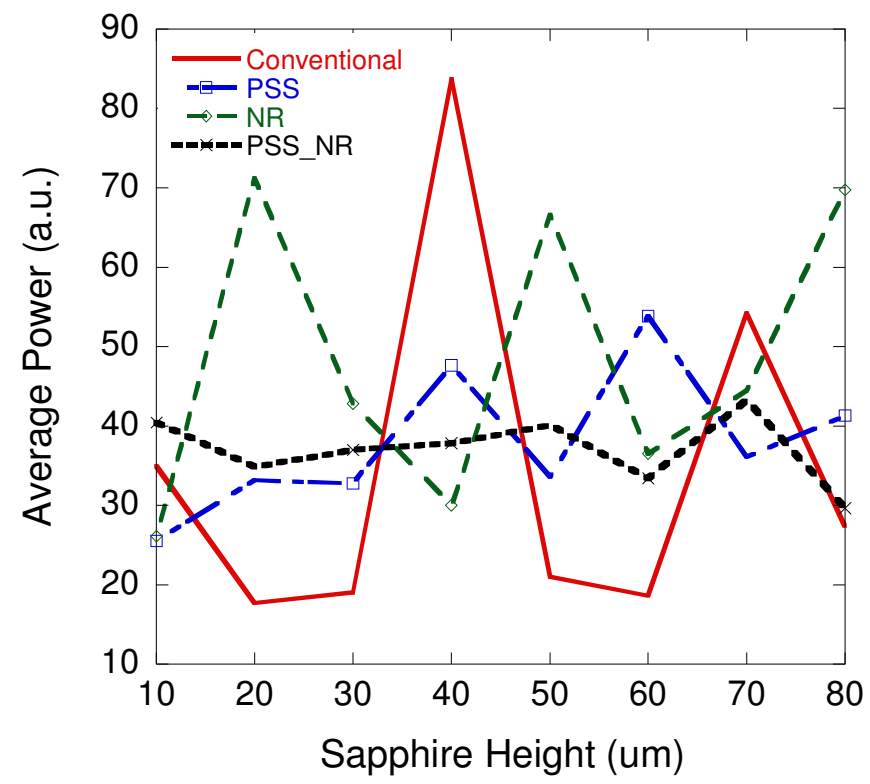

Figure 6.10 Average power vs. sapphire substrate height for four structures with a bottom layer Ag reflector.

\subsection{Conclusion}

This chapter has both an instructional and research component. The instructional component teaches how to properly use a metal such as silver (Ag) in Rsoft's FullWAVE software. The key elements include defining the dispersion terms of the material, turning on Dispersion/Nonlinearity, and using the correct simulation parameters required by FDTD analysis. The research portion investigates the use of Ag between the sapphire substrate and undoped GaN layer as well as below the sapphire substrate. The results show that a conventional LED with a reflection layer at the bottom can increase light extraction as much as $160 \%$ and decrease as much as $45 \%$ compared to a conventional LED with no reflection layer. The same structure extracts the least and the most amount of light in this entire study by changing sapphire substrate thickness from $20 \mu \mathrm{m}$ to $40 \mu \mathrm{m}$. Figure 6.10 shows light extraction periodically increasing and decreasing as a function sapphire height similar to the results in Chapter 4. Very little research focuses on light 
extraction as a function of mirror position. Further analysis should focus on the mirror position as well as mirror shape. Research shows that both convex and concave shaped silver mirrors can be used to improve light extraction efficiency. ${ }^{35}$ To improve the accuracy of the simulation, time monitors should be placed on top and sides of the LED structure. This method effectively monitors all possible areas light can escape the LED. 


\section{CHAPTER 7: CONCLUSION AND FUTURE WORK}

\subsection{Overall Conclusion}

The scope of this research covers the effects of nano-scale top gratings, patterned sapphire substrates, $\mathrm{SiO}_{2}$ nanorod arrays, and Ag reflection layers. All simulations use the FDTD method to analyze the light extraction properties of each LED. Chapter 3 investigates the effects of changing top grating shape, period, fill factor, and depth. The results show that cylindrical and conical gratings can achieve a $17.8 \%$ increase in light extraction. The cylindrical case achieves this improvement with a grating period of $100 \mathrm{~nm}$, grating depth of $200 \mathrm{~nm}$, and fill factor of $33 \%$. The conical case achieves this improvement with a grating period of $100 \mathrm{~nm}$, grating depth $200 \mathrm{~nm}$, and fill factor of $66 \%$. Unfortunately the results of this study do not match similar results from Peking University $^{25}$ and Kyungpook Nationional University. ${ }^{24}$ The results of these three studies could differ for several reasons. First, the simulations could have been set up differently. Our simulation does not take into account the reflections or absorption from the MQW, p-GaN layer, or the sapphire substrate. Second, different methods of simulation could reveal different results. Peking University uses RCWA to analyze transmission percentage, while we use FDTD to analyze average power of light extracted. Finally, our simulation only measures light transmittied through the top. Alternatively, light could be transmitted through the sides of the LED wall.

Chapter 4 studies the differences in using an ITO based top grating into epoxy rather than GaN to air. In this section we confirm the results of Peking University's RCWA simulations and show that increasing the critical angle of a device increases light extraction. First, increasing the critical angle from 27.5 degrees to 38.5 degrees by 
changing the grating material from GaN to ITO and the surrounding medium from air to epoxy improves light extraction by $40 \%$. Next, altering the ITO grating period shows low light transmission occurs in the $100-690 \mathrm{~nm}$ range and high transmission occurs for larger grating periods in the $690-1500 \mathrm{~nm}$ range. These results agree with those of Peking University which showed optimal light transmission with a grating period of 1130nm. Last, we show that Ag reflection layers have the potential to vastly improve or degrade light extraction depending on the position of the Ag layer.

Finally, Chapter 5 and Chapter 6 optimize light extraction for a structure containing a patterned sapphire substrate and a $\mathrm{SiO}_{2}$ nanorod array. First, optimizing the period and width of the patterned sapphire substrate improves light extraction by $47.9 \%$ for a width of $1.3 \mu \mathrm{m}$ and period of $2 \mu \mathrm{m}$. Next, we optimize the z-position of the $\mathrm{SiO}_{2}$ nanorod array to $7.1 \mu \mathrm{m}$. A combination of $\mathrm{z}$ position and a PSS period of $3 \mu \mathrm{m}$ improves light extraction by $51.8 \%$ compared to a conventional LED. Last of all, we include an Ag reflection layer between the sapphire substrate and $\mathrm{n}-\mathrm{GaN}$ layer as well as below the sapphire substrate. We find that the position of the reflection layer has major effects on light extraction. For example, changing the sapphire substrate width of a conventional LED with an Ag layer from $20 \mu \mathrm{m}$ to $40 \mu \mathrm{m}$ increases average power from 17.7 (a.u.) to 83.7 (a.u.). From these simulations we find a maximum increase in light extraction of $160 \%$ for a conventional LED with a sapphire substrate width of $40 \mu \mathrm{m}$ and an Ag reflection layer compared to a conventional LED without the Ag reflection layer.

\subsection{Future Work}

The effect of changing an Ag reflector's position remains the most significant finding of this research. Very few studies exist focusing on sapphire substrate thickness and its 
effect on light extraction. One study in particular shows that changing Ag position from the bottom of sapphire to the sapphire GaN interface increases light extraction. They claim increased light extraction is a result of light passing through less bonded interfaces. ${ }^{26}$ To confirm the results of our study, we need to conduct more simulations and further our collaboration with Peking University. First, new simulations should include time monitors on all sides of the LED. The use of multiple time monitors provides more information on where light exits the device. Next, simulations should also investigate light extraction efficiency for concave and convex mirror shapes. Literature has shown the use of highly reproducible roughened reflectors taking both of these shapes. ${ }^{35}$ Finally, these LED structures should be fabricated and tested. Physical measurements will support or deny the results of our study and reveal the usefulness of such simulations. 


\section{APPENDIX A - FINITE DIFFERENCE TIME DOMAIN METHOD}

\section{Solution to FDTD Method}

To obtain the FDTD equations, we begin with the curl equations of Faraday and Ampere's Law shown in equation A.1.

$$
\begin{aligned}
& \nabla \times \bar{E}=-\mu \frac{\partial \bar{H}}{\partial t} \\
& \nabla \times \bar{H}=\sigma \bar{E}+\varepsilon \frac{\partial \bar{E}}{\partial t}
\end{aligned}
$$

Next, we convert the vector differential equations into three scalar differential equations by executing the curl operation on the LHS of both equations as seen in equation A.2.

$$
\begin{aligned}
& \nabla \times \bar{E}=\hat{x}\left(\frac{\partial E_{z}}{\partial y}-\frac{\partial E_{y}}{\partial z}\right)-\hat{y}\left(\frac{\partial E_{z}}{\partial x}-\frac{\partial E_{x}}{\partial z}\right)+\hat{z}\left(\frac{\partial E_{y}}{\partial x}-\frac{\partial E_{x}}{\partial y}\right) \\
& \nabla \times \bar{H}=\hat{x}\left(\frac{\partial H_{z}}{\partial y}-\frac{\partial H_{y}}{\partial z}\right)-\hat{y}\left(\frac{\partial H_{z}}{\partial x}-\frac{\partial H_{x}}{\partial z}\right)+\hat{z}\left(\frac{\partial H_{y}}{\partial x}-\frac{\partial H_{x}}{\partial y}\right)
\end{aligned}
$$

Now, by equating the scalar differential equations to the RHS of the curl equations, we obtain six individual time and space derivatives in equation A.3 and A.4.

$$
\begin{aligned}
& \frac{\partial H_{x}}{\partial t}=\frac{-1}{\mu}\left(\frac{\partial E_{z}}{\partial y}-\frac{\partial E_{y}}{\partial z}\right) \\
& \frac{\partial H_{y}}{\partial t}=\frac{-1}{\mu}\left(\frac{\partial E_{x}}{\partial z}-\frac{\partial E_{z}}{\partial x}\right) \\
& \frac{\partial H_{z}}{\partial t}=\frac{-1}{\mu}\left(\frac{\partial E_{y}}{\partial x}-\frac{\partial E_{x}}{\partial y}\right) \\
& \frac{\partial E_{x}}{\partial t}=\frac{1}{\varepsilon}\left[\left(\frac{\partial H_{z}}{\partial y}-\frac{\partial H_{y}}{\partial z}\right)-\sigma E_{x}\right]
\end{aligned}
$$




$$
\begin{aligned}
& \frac{\partial E_{y}}{\partial t}=\frac{1}{\varepsilon}\left[\left(\frac{\partial H_{x}}{\partial z}-\frac{\partial H_{z}}{\partial x}\right)-\sigma E_{y}\right] \\
& \frac{\partial E_{z}}{\partial t}=\frac{1}{\varepsilon}\left[\left(\frac{\partial H_{y}}{\partial x}-\frac{\partial H_{x}}{\partial y}\right)-\sigma E_{z}\right]
\end{aligned}
$$

In order to make these equations useful, we must discretize both time and space. First we discretize space using the Yee cell shown in Figure A.1. The three components $(\Delta \mathrm{x}, \Delta \mathrm{y}$, and $\Delta \mathrm{z}$ ) represent the height, width, and depth that define the volume of one cell. Notice that the components of the E-field, shown in black, lie on the edge of the cell, while the components of the H-field, shown in red, lie on the faces of the cell. Also, the electric field for the next cell shares an edge with the primary cell. We title this component of the electric field $E_{y}(i+1, j, k)$ to distinguish it from $E_{y}(i, j, k)$. Using the Yee cell, we can rewrite the spatial differential equation shown in Equation A.5. 


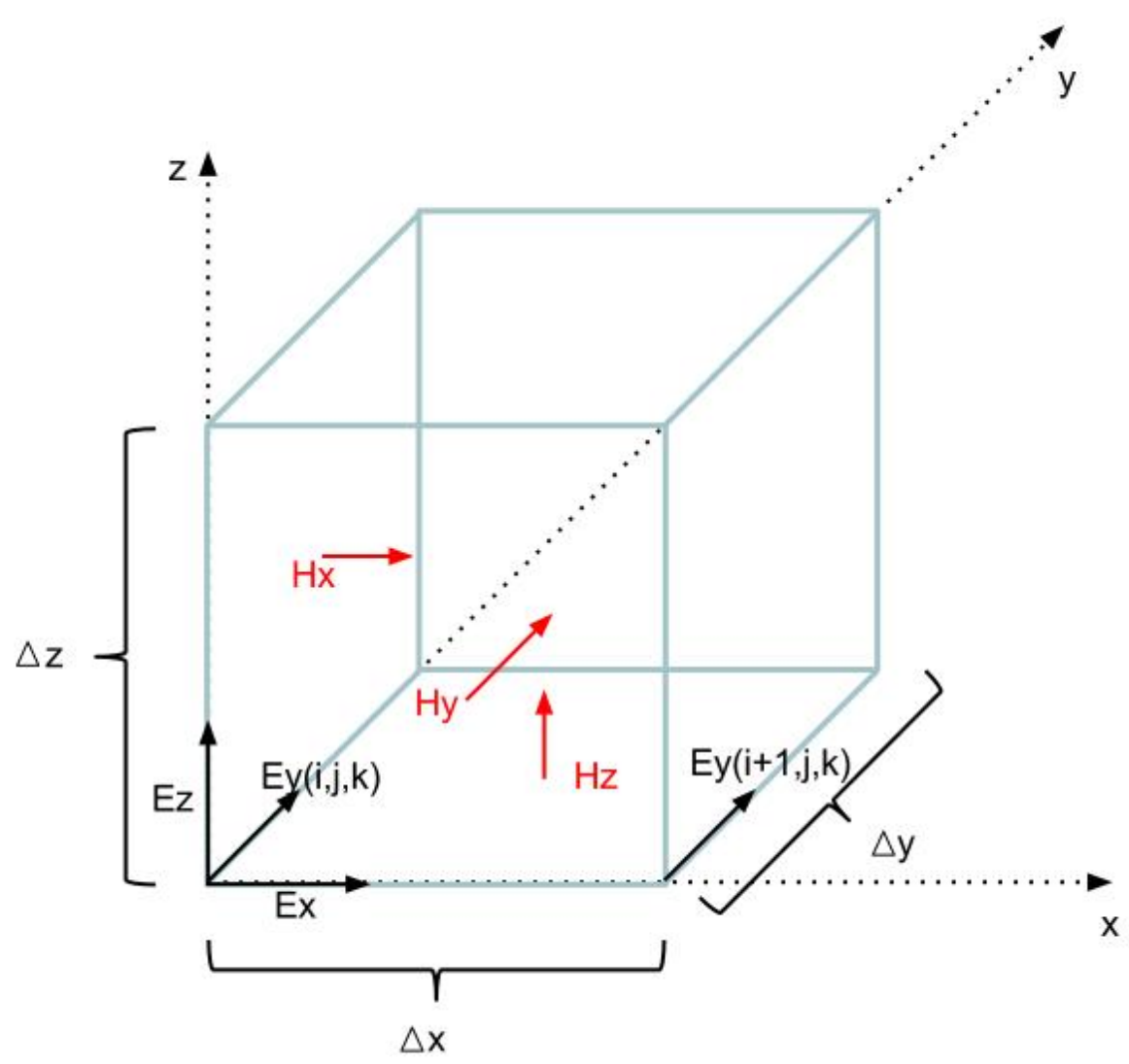

Figure A.1 Yee Cell.

$\frac{\partial E_{y}}{\partial x}-\frac{\partial E_{x}}{\partial y}=\frac{E_{y}(i+1, j, k)-E_{y}(i, j, k)}{\Delta x}-\frac{E_{x}(i, j+1, k)-E_{x}(i, j, k)}{\Delta y}$

The next step discretizes time. Figure A.2 shows the order in which the FDTD method calculates the E-field and $\mathrm{H}$-field as time passes. The letter $\mathrm{n}$ represents the $\mathrm{n}^{\text {th }}$ time step, a full time step occurs between $E^{n}$ and $E^{n+1}$. Every half of a time step, the calculation switches from the electric field to the magnetic field, often called the "leapfrog" method. ${ }^{36}$ Time discretization allows the time based differential equation to be rewritten as equation A.6. 


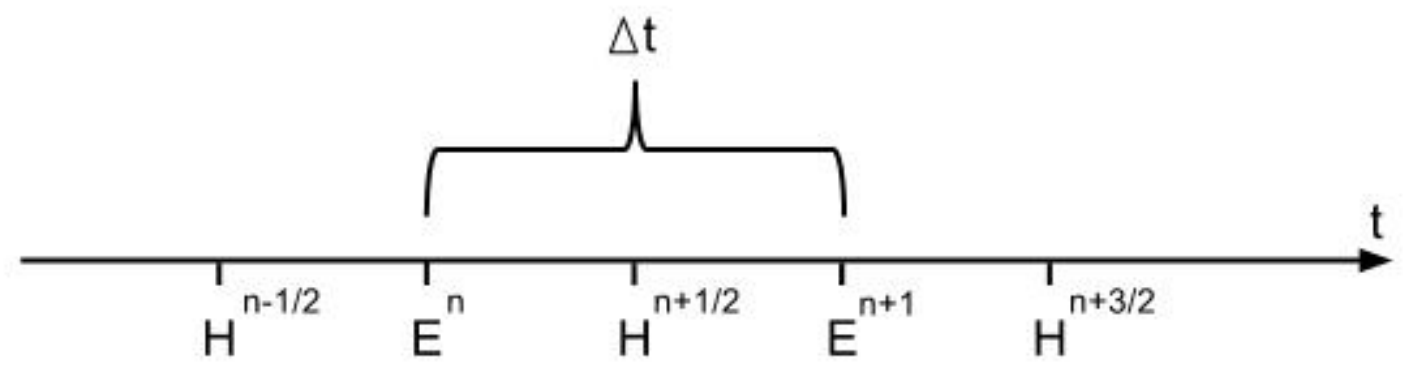

Figure A.2 Discretization of time domain.

$\frac{\partial H_{z}}{\partial t}=\frac{H_{z}^{n+\frac{1}{2}}(i, j, k)-H_{z}^{n-\frac{1}{2}}(i, j, k)}{\Delta t}$

A.6

Now that both time and space have been discretized, we can rewrite the entire difference equation. Notice that future values, such as $\mathrm{H}_{\mathrm{z}}{ }^{\mathrm{n}+1 / 2}$ and $\mathrm{E}_{\mathrm{y}}{ }^{\mathrm{n}+1}$, can be obtained from these equations.

$$
\begin{aligned}
& \frac{H_{x}^{n+\frac{1}{2}}(i, j, k)-H_{x}^{n-\frac{1}{2}}(i, j, k)}{\Delta t}=\frac{-1}{\mu}\left[\frac{E_{z}(i, j+1, k)-E_{z}(i, j, k)}{\Delta y}-\frac{E_{y}(i, j, k+1)-E_{y}(i, j, k)}{\Delta z}\right] \\
& \frac{H_{y}^{n+\frac{1}{2}}(i, j, k)-H_{y}^{n-\frac{1}{2}}(i, j, k)}{\Delta t}=\frac{-1}{\mu}\left[\frac{E_{x}(i, j, k+1)-E_{x}(i, j, k)}{\Delta z}-\frac{E_{z}(i+1, j, k)-E_{z}(i, j, k)}{\Delta x}\right] \\
& \frac{H_{z}^{n+\frac{1}{2}}(i, j, k)-H_{z}^{n-\frac{1}{2}}(i, j, k)}{\Delta t}=\frac{-1}{\mu}\left[\frac{E_{y}(i+1, j, k)-E_{y}(i, j, k)}{\Delta x}-\frac{E_{x}(i, j+1, k)-E_{x}(i, j, k)}{\Delta y}\right]
\end{aligned}
$$




$$
\begin{aligned}
& \frac{E_{x}^{n+1}(i, j, k)-E_{x}^{n}(i, j, k)}{\Delta t}=\frac{1}{\varepsilon}\left[\left(\frac{H_{z}(i, j+1, k)-H_{z}(i, j, k)}{\Delta y}-\frac{H_{y}(i, j, k+1)-H_{y}(i, j, k)}{\Delta z}\right)-\sigma E_{x}^{n}\right] \\
& \frac{E_{y}^{n+1}(i, j, k)-E_{y}^{n}(i, j, k)}{\Delta t}=\frac{1}{\varepsilon}\left[\left(\frac{H_{x}(i, j, k+1)-H_{x}(i, j, k)}{\Delta z}-\frac{H_{z}(i+1, j, k)-H_{z}(i, j, k)}{\Delta x}\right)-\sigma E_{y}^{n}\right] \\
& \frac{E_{x}^{n+1}(i, j, k)-E_{x}^{n}(i, j, k)}{\Delta t}=\frac{1}{\varepsilon}\left[\left(\frac{H_{y}(i+1, j, k)-H_{y}(i, j, k)}{\Delta x}-\frac{H_{x}(i, j+1, k)-H_{x}(i, j, k)}{\Delta y}\right)-\sigma E_{z}^{n}\right]
\end{aligned}
$$




\section{APPENDIX B - FULLWAVE CAD ENVIRONMENT}

\section{CAD Environment}

Rsoft's CAD environment allows the user to draw several geometrical shapes in both 2-D and 3-D. Figure B.1 shows an example of the CAD environment. The toolbar to the left contains icons used to choose different components to add such as a lens, circle, arc, polygon, or just a plain segment.

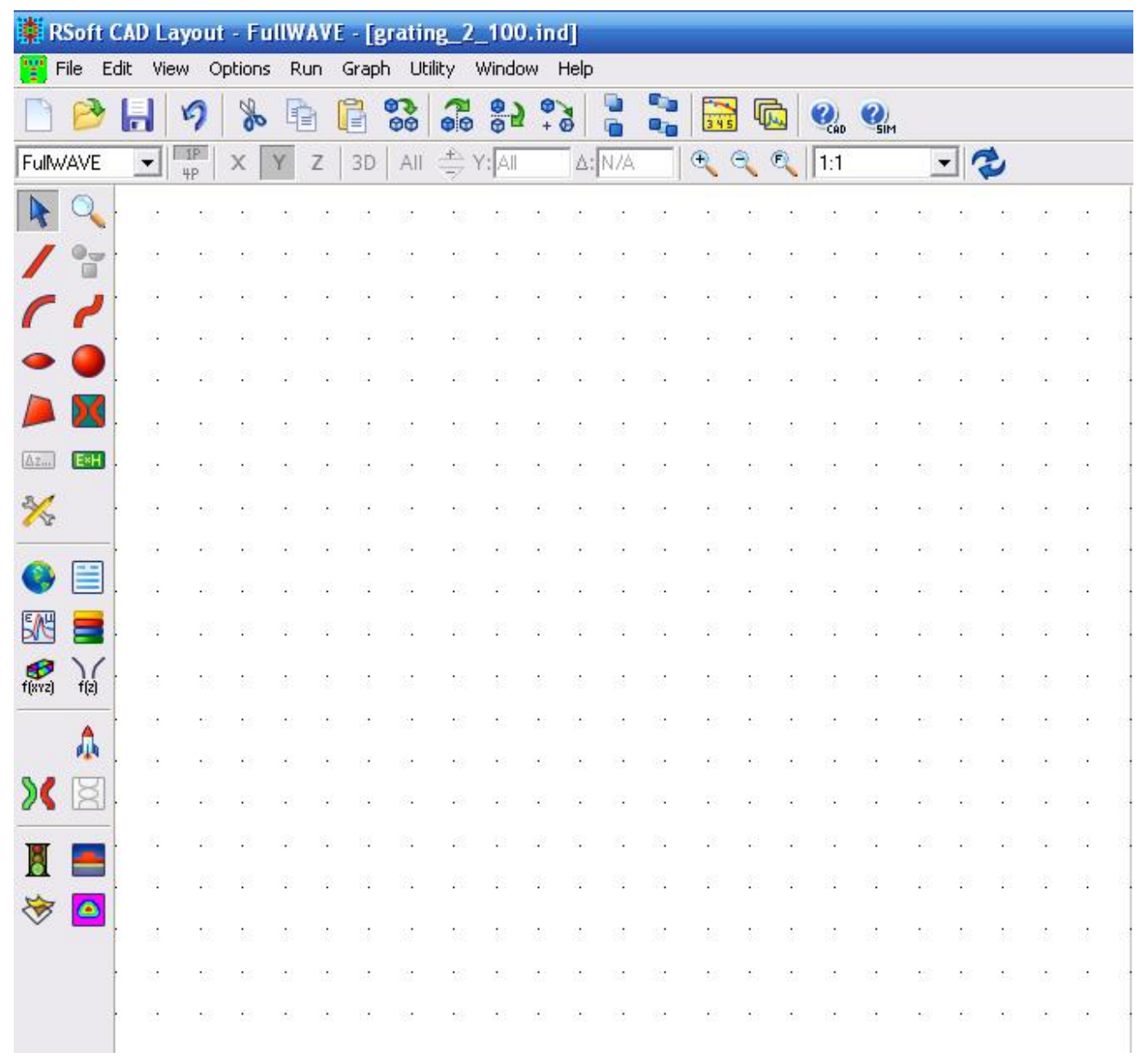

Figure B.1 Rsoft CAD environment.

Right clicking on a previously drawn segment brings up the properties of the segment, some of which include: material properties, refractive index, merge priority, component 
width, component height, 3D structure type, and component referencing. These properties, among others, make it simple to control the shape and placement of a segment. Before using these properties, it is helpful to know how to create variables. Clicking on the Edit Symbols symbol brings up the Symbols Table Editor. Here you can create and define variables such as the height and width of a layer in an LED. This is a particularly good way to define the period of a grating. For example using the Symbol Table Editor, create a new symbol and name it grating period. Define the value as 0.5 (this value corresponds to $500 \mathrm{~nm}$ ). Next click on a segment and define Component Width as grating_period/2 and set the $\mathrm{x}$ and $\mathrm{z}$ position to zero. Create a new segment and again define the Component Width as grating_period/2 and set the $\mathrm{x}$ position to grating_period/2 and $\mathrm{z}$ position to zero. Continue this pattern for several segments increasing the $\mathrm{x}$ position by half of the period each time. The created grating now has a variable grating period. Changing the value of grating period from 0.5 to 0.7 automatically changes the period of the grating from $500 \mathrm{~nm}$ to $700 \mathrm{~nm}$. Using this method, several different grating periods can quickly be simulated. 


\section{PUBLISHED WORKS}

[1] Greg Chavoor, Doug Cattarusa, Xiaomin Jin, Xing-Xing Fu, Xiang-Ning Kang, Bei Zhang, and Guo-Yi Zhang, "Optical Mode Pattern Study of GaN LEDs with and without Top Nano-Gratings", The SPIE Photonic West 2012, the Moscone Center, San Francisco, CA, January 21-26, 2012.

[2] Greg Chavoor, Xiaomin Jin, Ashton Ellaboudy, Xing-Xing Fu, Xiang-Ning Kang, Bei Zhang, and Guo-Yi Zhang, "Light Extraction Improvement of GaN LEDs using Nano-scale Top Transmission Gratings", The SPIE Optical Engineering + Applications, 21-25 August 2011 in San Diego, California , 2011.

[3] Ashton Ellaboudy, Michael James Marshall, Greg Chavoor, and Xiaomin Jin, "Study of silicon solar cell double triangular nano-gratings", The SPIE Photonic West 2012, the Moscone Center, San Francisco, CA, January 21-26, 2012.

[4] Xiaomin Jin, Ashton Ellaboudy, and Greg Chavoor, "Improvement of Solar Cell Efficiency Using Nano-scale Top and Bottom Grating”, SPIE Optical Engineering + Applications, 21-25 August 2011, San Diego, California, 2011.

[5] K. Lal, G. Chavoor, and X. Jin, "Wavelength Dependence of Transverse Mode Coupling With/Without E-block of GaN Laser Cavity," IEEE Photonics Journal, vol. 3, No. 6, pp. $1206-1213,2011$.

[6] Greg Chavoor, Xiaomin Jin, Fu XingXing, XiangNing Kang, and GuyiZhang, "Position of Ag Reflection Layer and its Effect on GaN-Based LED Light Extraction Efficiency," IEEE Photonic Tech. Lett, in Preparation. 


\section{REFERENCES}

[1] VDE, "Phase out of incandescent light bulbs (Edison light bulb)" Web. $<$ http://www.vdeglobalservices.com/en/news/Pages/2009-03.aspx>

[2] Navigant Consulting, Inc., "Energy Savings Potential of Solid-State Lighting in General Illumination Applications 2010 to 2030.” U.S. Energy Information Administration (EIA). Web. 05 Feb. 2012. $<$ http://apps1.eere.energy.gov/buildings/publications/pdfs/ssl/ssl_energy-savingsreport_10-30.pdf>.

[3] "Cree Sets New R\&D Performance Record with 254 Lumen-Per-Watt Power LED." Cree News: (12Apr2012). Web. 10 May 2012. <http://www.cree.com/news-andevents/cree-news/press-releases/2012/april/120412-254-lumen-per-watt>.

[4] S. Nakamura, M. Senoh, T. Mukai, "High-power InGaN/GaN doubleheterostructure violet light emitting diodes," Applied Physics Letters 62(19), pp. 2390-2392, May1993.

[5] Krames, M.R., Shchekin, O.B., and Mueller-Mach, R., "Status and Future of HighPower Light-Emitting Diodes for Solid-State Lighting," Journal of Display Technolog, 3 (2), 160-175 (2007).

[6] Czernecki, S., Krukowski, G., and Targowski, P., "Strain-compensated AlGaN/GaN/InGaN cladding layers in homoepitaxial nitride devices," Applied Physics Letters, pp 231914 (2007).

[7] Nakamura et al., "Light-Emitting Gallium Nitride-Based Compound Semiconductor Device.” US Patent 5,578,839, issued Nov. 26, 1996. 
[8] S. Nakamura, M. Senoh, T. Mukai, "High-power InGaN/GaN doubleheterostructure violet light emitting diodes," Applied Physics Letters 62(19), pp. 2390-2392, May1993.

[9] Shuji Nakamura, "Crystal Growth Method for Gallium Nitride-Based Compound Semiconductor," US Patent 5,290,393, issued Mar. 1, 1994.

[10] S. Nakamura, Y. Harada, M. Seno, "Novel metalorganic chemical vapor deposition system for GaN growth,” Appl. Phys. Lett. 58(18), pp. 2021-2023, May 1991.

[11] Neamen, D.A., "Semiconductor Physics and Devices: Basic Principles, Fourth Edition," McGraw-Hill (2012), 648-651.

[12] Gardner, N. F., and Chui, H.C., "1.4x efficiency improvement in transparentsubstrate $\left(\mathrm{Al}_{\mathrm{x}} \mathrm{Ga}_{1-\mathrm{x}}\right)_{0.5} \mathrm{In}_{0.5} \mathrm{P}$ light-emitting diodes with thin $(\leq 2000 \AA)$ active regions," Appl. Phys. Lett., 74(15), 2230-2232 (1999).

[13] Bao, K., Kang, X.N., and Zhang, B., "Improvement of light extraction from GaNbased thin-film light-emitting diodes by patterning undoped GaN using modified laser lift-off," Applied Physics Letters, 92, pp. 141104 (2008).

[14] Dai, T., Kang, X.N., and Zhang, B., "Study and formation of 2D microstructures of sapphire by focused ion beam milling," Microelectronic Engineering 85, pp. 640645 (2008).

[15] Kim, B.J., Jung, H., and Kim, S.H., "GaN-Based Light-Emitting Diode with ThreeDimensional Silver Reflectors,” IEEE Photonics Technology Letters, 21(11), 700702 (2009).

[16] Palais, J.C., " Fiber Optic Communications: Fifth Edition,” Pearson Prentice Hall, 2005. 
[17] Huang, X.H., Liu, J. P., and F, Y.Y., "Effect of Patterned Sapphire Substrate Shape on Light Output Power of GaN-Based LEDs," IEEE Photonics Technology Letters, 23 (14), 944-946 (2011).

[18] Dai, T., and Kang, X., "Study and formation of 2D microstructures of sapphire by focused ion beam milling,” Microelectronic Engineering, 85(3), 640-645 (2008).

[19] Kim, S., and Lee, K., "Fabrication of photonic crystal structures on light emitting diodes by nanoimprint lithography," Nanotechnology, 18(5), 1150(2007).

[20] Trieu, S., and Jin, X., "Study of Top and Bottom Photonic Gratings on GaN LED with Error Grating Models,” IEEE Journal of Quantum Electronics, 46（10）, 896901 (2010).

[21] Bao, K. and Kang, X.N., "Improvement of Light Extraction from Patterned Polymer Encapsulated GaN-Based Flip-Chip Light-Emitting Diodes by Imprinting,” IEEE Photonics Technology Letters, 19(22), 1840-1842 (2007).

[22] Ryu, H. and Shim, J., "Structural Parameter Dependence of Light Extraction Efficiency in Photonic Crystal InGaN Vertical Light-Emitting Diode Structures," IEEE Journal of Quantum Electronics, 46(5), 714-720 (2010).

[23] Lin, Y. Z. and Kong, F.M., "FDTD Study on the Improvement of Optical Transmission through Metallic Periodic Nano Structure,” Proc. OptoElectronics and Communications Conference, 1-2 (2009).

[24] Ju, Y.G., Lee, B.W., "Analysis of the Effect of Surface Texture in Light-Emitting Diodes Based on a Finite-Difference-Time-Domain Method,” Japanese Journal of Applied Physics, 46(8A), 5153-5156 (2007). 
[25] Fu, X.X., Zhang, B., and Kang, X. N., "Lattice Constant Effects of Photonic Crystals on the Extraction of Guided Mode of GaN Based Light Emitting Diodes," Science China, 54(1), 1-5 (2011).

[26] Peng, W.C., and Wu, Y.C.S, "Enhance performance of an InGaN-GaN light emitting diode by roughening the undoped-GaN surface and applying a mirror coating to the sapphire substrate,” Applied Physics Letters, 88, pp. 181117 (2006).

[27] Gibart, P., "Metal Organic Vapour Phase Epitax of GaN Lateral Overgrowth," Rep. Prog. Physics, 67(5), (2004).

[28] Huang, H.W., Huange, J.K., and Lin, C.H., "Efficiency Improvement of GaNBased LEDs with a $\mathrm{SiO}_{2}$ Nanorod Array and a Patterned Sapphire Substrate," IEEE Electron Device Letters, 31(6), 582-584 (2010).

[29] Chiu, C.H., Yen, H.H., Chao, C.L., "Nanoscale Epitaxial Lateral Overgrowth of GaN Based Light-Emitting Diodes on a $\mathrm{SiO}_{2}$ Nanorod-array Patterened Sapphire Template,” Applied Physics Letters, 93 (81108), 1-3 (2008).

[30] Charlton, M.D.B, Zoorob, and M.E., Lee, T., "Photonic Quasi-Crystal LEDs: Design, modeling and optimization," Proceedings of SPIE, 64860R (2007).

[31] Ju, I., Kwon, Y., and Shin, C.S., "High-Power GaN-Based Light-Emitting Diodes Using Thermally Stable and Highly Reflective Nano-Scaled Ni-Ag-Ni-Au Mirror," IEEE Photonics Technology Letters, 23(22), 1685-1687 (2011).

[32] Jeong, T., Kim, K.H., and Lee, H.H., "Enhance Light Output Power of GaN-Based Vertical Light-Emitting Diodes by Using Highly Reflective ITO-Ag-Pt Reflectors," IEEE Photonics Technology Letters, 20 (23), 1932-1934 (2008). 
[33] Miller, M.A., Lin, S.K., and Mohney, S.E., "V/AI/V/Ag contacts to n-GaN and nAlGaN,” Journal of Applied Physics, 104 (6), 064508-1-064508-4 (2008).

[34] Shei, S.C., Lai, W.C., and Sheu, J.K., “The Output Power Enhancements of GaNBased Blue Light Emitting Diodes with Highly Reflective Ag/Cr/Au Trilayer Omnidirectional Reflective Electrode Pads,” Japanese Journal of Applied Physics, 48, 102103-1-102103-4 (2009).

[35] Kim, B.J., Jung, H., and Bang, J., "GaN-Based Light-Emitting Diode With ThreeDimensional Silver Reflectors,” IEEE Photonics Technology Letters, 21(11), 700702 (2009).

[36] Krishnaiah, K.M. and Railton, C.J., “A Stable Subgridding Algorithm and Its Application to Eigenvalue Problems," IEEE Transactions of Microwave Theory and Techniques, 47(5), 620-628 (1999). 